\title{
Radical Transfer Hydroamination with Aminated Cyclohexadienes Using Polarity Reversal Catalysis: Scope and Limitations
}

\author{
Joyram Guin, ${ }^{\dagger}{ }^{\ddagger}$ Christian Mück-Lichtenfeld, ${ }^{\ddagger}$ Stefan Grimme, ${ }^{*}{ }^{\star}$ and Armido Studer ${ }^{* *}$
}

Fachbereich Chemie, Organisch-Chemisches Institut, Westfälische Wilhelms-Universität, Corrensstrasse 40, 48149 Münster, and NRW Graduate School of Chemistry, Westfälische WilhelmsUniversität, Corrensstrasse 36, 48149 Münster.

studer@uni-muenster.de

† Organisch-Chemisches Institut, ${ }^{\dagger}$ NRW Graduate School of Chemistry.

Full reference 15 of the paper:

TURBOMOLE V5-7: Ahlrichs, R.; Bär, M.; Baron, H.-P.; Bauernschmitt, R.; Böcker, S.; Deglmann, P.; Ehrig, M.; Eichkorn, K.; Elliott, S.; Furche, F.; Haase, F.; Häser, M.; Horn, H.; Hättig, C.; Huber, C.; Huniar, U.; Kattannek, M.; Köhn, A.; Kölmel, C.; Kollwitz, M.; May, K.; Ochsenfeld, C.; Öhm, H.; Patzelt, H.; Rubner, O.; Schäfer, A.; Schneider, U.; Sierka, M.; Treutler, O.; Unterreiner, B.; Arnim, M. V.; Weigend, F.; Weis, P.; Weiss, H. Universität Karlsruhe 2005.

\section{Experimental Section:}

General: All reactions involving air- or moisture-sensitive reagents or intermediates were carried out in hot dried glassware under an argon atmosphere. Tetrahydrofuran (THF) was freshly distilled from potassium under argon. Diethylether $\left(\mathrm{Et}_{2} \mathrm{O}\right)$ was freshly distilled from $\mathrm{K} / \mathrm{Na}$ under argon. Dichloromethane $\left(\mathrm{CH}_{2} \mathrm{Cl}_{2}\right)$ was freshly distilled from phosphorus $(\mathrm{V})$ oxide $\left(\mathrm{P}_{2} \mathrm{O}_{5}\right)$. Triethylamine were distilled from $\mathrm{CaH}_{2}$ and stored over molecular sieve $4 \AA$. All other solvents and reagents were purified according to standard procedures or were used as received from Aldrich, Fluka or Acros. ${ }^{1} \mathrm{H}-$ and ${ }^{13} \mathrm{C}$ NMR spectra were recorded on a Bruker AMX 400, ARX 300. TLC was performed using Merck silica gel 60 F254 plates; detection with UV or dipping into a solution of $\mathrm{KMnO}_{4}\left(1.5 \mathrm{~g}\right.$ in $400 \mathrm{~mL} \mathrm{H}_{2} \mathrm{O}, 5 \mathrm{~g}$ 
$\left.\mathrm{NaHCO}_{3}\right)$ or a solution of $\mathrm{Ce}\left(\mathrm{SO}_{4}\right)_{2} \times \mathrm{H}_{2} \mathrm{O}(10 \mathrm{~g})$, phosphormolybdic acid hydrate $(25 \mathrm{~g})$, concentrated $\mathrm{H}_{2} \mathrm{SO}_{4}(60 \mathrm{~mL})$, and $\mathrm{H}_{2} \mathrm{O}(940 \mathrm{~mL})$, followed by heating. Flash column chromatography (FC) was performed using Merck or Fluka silica gel $60(40-63 \mu \mathrm{m})$ applying a pressure of about 0.4 bar. IR spectra were recorded on a Bruker IFS-28 and a Digilab FTS 4000 spectrophotometer. Mass spectra were recorded on a Finnigan MAT 4200S, a Bruker Daltonics Micro Tof, a Waters-Micromass Quatro $L C Z$ (ESI); and peaks are given in $\mathrm{m} / z$ (\% of basis peak).

\section{General Procedure (1) for the Synthesis of Enamidones from 1,3-Diketones:}

A catalytic amount of an acid (p-toluenesulfonic acid; TsOH) and the amide (1.25 equiv.) was added to a stirred solution of acetylacetone (1.0 equiv.) in toluene. The mixture was heated under reflux using a Dean-Stark apparatus. After 6-8 h the reaction mixture was allowed to cool to r.t. The mixture was washed with saturated $\mathrm{NaHCO}_{3}$ solution and water, dried over $\mathrm{MgSO}_{4}$, concentrated under reduced pressure. The solid crude material was purified by $\mathrm{FC}\left(\mathrm{SiO}_{2}\right)$ (pentane / $t$-butylmethylether) to afford the desire corresponding keto-amide.

$\mathrm{N}$-((Z)-1-Methyl-3-oxobut-1-enyl)acetamide: Prepared according to the general procedure (1) using acetylacetone $(5.0 \mathrm{~g}, 50 \mathrm{mmol})$, acetamide $(3.7 \mathrm{~g}, 63 \mathrm{mmol})$, PTSA $(0.5 \mathrm{~g})$ in toluene $(180 \mathrm{ml})$. Purification by FC (pentane / MTBE 5:1) provided $N$-((Z)-1-Methyl-3-oxobut-1-enyl)acetamide as a white solid ${ }^{\mathrm{a}}$ (5.0 g, $72 \%$ ). IR (neat) 3125br.w, 1727s, 1632s, 1576s, 1467m, 1421s, 1363s, 1246s, $1211 s, 1179 s, 1035 s, 981 s, 956 s, 925 w, 835 m, 795 s \mathrm{~cm}^{-1} .{ }^{1} \mathrm{H}$ NMR $\left(300 \mathrm{MHz}, \mathrm{CDCl}_{3}\right) \delta 2.11(s, 3 \mathrm{H}$, $\left.\mathrm{CH}_{3}\right), 2.12\left(s, 3 \mathrm{H}, \mathrm{CH}_{3}\right), 2.33\left(d, 3 \mathrm{H}, J=0.9 \mathrm{~Hz}, \mathrm{NHCCH}_{3}\right), 5.30$ ( $s$, br., $1 \mathrm{H}, \mathrm{CH}$-vinylic), 11.97 ( $s$, br., $1 \mathrm{H}, \mathrm{NH}) .{ }^{13} \mathrm{C}$ NMR $\left(75 \mathrm{MHz}, \mathrm{CDCl}_{3}\right) \delta 21.71\left(\mathrm{CH}_{3}\right), 25.30\left(\mathrm{CH}_{3}\right), 30.39\left(\mathrm{CH}_{3}\right), 105.22(\mathrm{CH}), 155.22$ (C), $169.65(\mathrm{C}), 199.57$ (C). HRMS (ESI) calculated for $\mathrm{C}_{7} \mathrm{H}_{11} \mathrm{NO}_{2} \mathrm{Na}\left([\mathrm{M}+\mathrm{Na}]^{+}\right)$: 164.0682. Found: 164.0683.

$\boldsymbol{N}$-((Z)-1-Methyl-3-oxobut-1-enyl)benzamide: Prepared according to the general procedure (1) using acetylacetone $(5.0 \mathrm{~g}, 50 \mathrm{mmol})$, benzamide $(9.8 \mathrm{~g}, 63 \mathrm{mmol})$, PTSA $(0.5 \mathrm{~g})$ in toluene $(180 \mathrm{ml})$. Purification by FC (pentane / MTBE 5:1) provided $N$-((Z)-1-Methyl-3-oxobut-1-enyl)benzamide as a white solid $^{\mathrm{a}}$ (7.7 g, 65\%). IR (neat) 3067br.w, 1688s, 1633s, 1588s, 1478s, 1432s, 1354s, 1262s, 1236s, $1195 m, 1093 m, 1025 m, 976 m, 897 m, 855 \mathrm{w} \mathrm{cm}^{-1} .{ }^{1} \mathrm{H}$ NMR $\left(300 \mathrm{MHz}, \mathrm{CDCl}_{3}\right) \delta 2.17\left(\mathrm{~s}, 3 \mathrm{H}, \mathrm{COCH}_{3}\right)$, $2.50\left(d, 3 \mathrm{H}, J=0.9 \mathrm{~Hz}, \mathrm{NHCCH}_{3}\right), 5.44(s, 1 \mathrm{H}, \mathrm{CH}$-vinylic), 7.57-7.44 ( $m, 3 \mathrm{H}, \mathrm{CH}$-aromatic), 8.01-7.98 ( $m, 2 \mathrm{H}, \mathrm{CH}$-aromatic), $10.86(s$, br., $1 \mathrm{H}, \mathrm{NH}) .{ }^{13} \mathrm{C} \mathrm{NMR}\left(75 \mathrm{MHz}, \mathrm{CDCl}_{3}\right) \delta 21.96\left(\mathrm{CH}_{3}\right), 30.39\left(\mathrm{CH}_{3}\right)$, $106.08(\mathrm{CH}), 127.87(\mathrm{CH}), 128.79(\mathrm{CH}), 132.53(\mathrm{CH}), 133.57(\mathrm{C}), 156.01(\mathrm{C}), 165.95(\mathrm{C}), 199.99(\mathrm{C})$. HRMS (ESI) calculated for $\mathrm{C}_{12} \mathrm{H}_{13} \mathrm{NO}_{2} \mathrm{Na}\left([\mathrm{M}+\mathrm{Na}]^{+}\right)$: 226.0838 . Found: 226.0825

2,6-Difluoro- $N$-((Z)-1-methyl-3-oxobut-1-enyl)benzamide: Prepared according to the general procedure (1) using acetylacetone $(5.0 \mathrm{~g}, 50 \mathrm{mmol})$, 2,6-difluorobenzamide $(9.9 \mathrm{~g}, 63 \mathrm{mmol})$, PTSA $(0.5$ $\mathrm{g})$ in toluene $(180 \mathrm{ml})$. Purification by FC (pentane / MTBE 5:1) provided 2,6-Difluoro- $N-((Z)-1$ methyl-3-oxobut-1-enyl)benzamide as a white solid (6.8 g, $57 \%$ ). IR (neat) 3101br.w, 1703s, 1644s, $1592 s, 1481 s, 1462 s, 1438 s, 1354 s, 1276 s, 1256 s, 1219 m, 1196 s, 1112 s, 1041 m, 1003 s, 984 m, 897 m$, $856 m, 810 s, 788 \mathrm{sm}^{-1} .{ }^{1} \mathrm{H}$ NMR $\left(300 \mathrm{MHz}, \mathrm{CDCl}_{3}\right) \delta 2.14\left(s, 3 \mathrm{H}, \mathrm{COCH}_{3}\right), 2.50(d, 3 \mathrm{H}, J=0.9 \mathrm{~Hz}$, $\left.\mathrm{NHCCH}_{3}\right), 5.44$ ( $s, 1 \mathrm{H}, \mathrm{CH}$-vinylic), 7.00-6.93 ( $m, 2 \mathrm{H}, \mathrm{CH}$-aromatic), 7.45-7.35 ( $m, 1 \mathrm{H}, \mathrm{CH}$-aromatic), $11.50(s$, br., $1 \mathrm{H}, \mathrm{NH}) .{ }^{13} \mathrm{C} \mathrm{NMR}\left(75 \mathrm{MHz}, \mathrm{CDCl}_{3}\right) \delta 22.01\left(\mathrm{CH}_{3}\right), 30.50\left(\mathrm{CH}_{3}\right), 106.75(\mathrm{CH}), 112.19$ $(d d, J=2.3 \mathrm{~Hz}, J=22.9 \mathrm{~Hz}, \mathrm{CH}), 114.26(t, J=19.1 \mathrm{~Hz}, \mathrm{C}), 132.44(t, J=10.3 \mathrm{~Hz}, \mathrm{CH}), 154.14(\mathrm{C})$, $159.53(\mathrm{C}), 159.96(d d, J=6.3 \mathrm{~Hz}, J=254.0 \mathrm{~Hz}, \mathrm{C}), 199.83$ (C). HRMS (ESI) calculated for $\mathrm{C}_{12} \mathrm{H}_{11} \mathrm{~F}_{2} \mathrm{NO}_{2} \mathrm{Na}\left([\mathrm{M}+\mathrm{Na}]^{+}\right): 262.0650$. Found: 262.0643 . 
4-Methoxy- $N$-((Z)-1-methyl-3-oxobut-1-enyl)benzamide: Prepared according to the general procedure (1) using acetylacetone $(5.0 \mathrm{~g}, 50 \mathrm{mmol})$, 4-methoxybenzamide $(9.4 \mathrm{~g}, 63 \mathrm{mmol})$, PTSA $(0.5 \mathrm{~g})$ in toluene $(180 \mathrm{ml})$. Purification by FC (pentane / MTBE 5:1) provided 4-Methoxy- $N$ - $((Z)-1$-methyl-3oxobut-1-enyl)benzamide as a white solid (7.8 g, $67 \%$ ). IR (neat) 3055br.w, 3001m, 1681s, 1640s, $1598 s, 1574 s, 1480 s, 1437 s, 1354 s, 1250 s, 1184 s, 1123 s, 1102 s, 1027 s, 980 m, 898 w, 863 s, 758 s \mathrm{~cm}^{-1}$. ${ }^{1} \mathrm{H}$ NMR $\left(300 \mathrm{MHz}, \mathrm{CDCl}_{3}\right) \delta 2.18\left(s, 3 \mathrm{H}, \mathrm{COCH}_{3}\right), 2.50\left(d, J=0.9 \mathrm{~Hz}, \mathrm{NHCCH}_{3}\right), 3.85(s, 3 \mathrm{H}$, $\mathrm{OCH}_{3}$ ), 5.42 ( $s$, br., $1 \mathrm{H}, \mathrm{CH}$-vinylic), 6.97-6.94 ( $\mathrm{m}, 2 \mathrm{H}, \mathrm{CH}$-aromatic), 8.00-7.97 ( $\mathrm{m}, 2 \mathrm{H}, \mathrm{CH}$-aromatic), $10.91(s$, br., $1 \mathrm{H}, \mathrm{NH}) .{ }^{13} \mathrm{C} \mathrm{NMR}\left(75 \mathrm{MHz}, \mathrm{CDCl}_{3}\right) \delta 21.96\left(\mathrm{CH}_{3}\right), 30.34\left(\mathrm{CH}_{3}\right), 55.42\left(\mathrm{CH}_{3}\right), 105.66$ $(\mathrm{CH}), 114.00(\mathrm{CH}), 125.87(\mathrm{C}), 129.95(\mathrm{CH}), 156.41(\mathrm{C}), 163.04(\mathrm{C}), 165.45(\mathrm{C}), 199.87(\mathrm{C})$. HRMS (ESI) calculated for $\mathrm{C}_{13} \mathrm{H}_{15} \mathrm{NO}_{3} \mathrm{Na}\left([\mathrm{M}+\mathrm{Na}]^{+}\right)$: 256.0944. Found: 256.0945.

1-((Z)-1-Methyl-3-oxobut-1-enyl)pyrrolidin-2-one: Prepared according to the general procedure (1) using acetylacetone (5.0 g, $50 \mathrm{mmol})$, 2-pyrrolidinone (4.4 g, $63 \mathrm{mmol})$, PTSA $(0.5 \mathrm{~g})$, toluene (180 ml). Purification by FC (pentane / MTBE 1:2) provided 1-((Z)-1-Methyl-3-oxobut-1-enyl)pyrrolidin-2-one as a white $\operatorname{solid}^{\mathrm{a}}$ (2.9 g, $35 \%$ ). IR (neat) 2971br.w, 2891w, 1711s, 1665s, 1567s, 1461w, 1419m, 1366s, $1313 s, 1179 s, 1124 m, 1029 m, 961 m, 843 w, 815 s \mathrm{~cm}^{-1} .{ }^{1} \mathrm{H}$ NMR $\left(300 \mathrm{MHz}, \mathrm{CDCl}_{3}\right) \delta 2.07-1.98(m, 2 \mathrm{H}$, $\left.\mathrm{CH}_{2}\right), 2.17\left(s, 3 \mathrm{H}, \mathrm{COCH}_{3}\right), 2.52\left(t, 2 \mathrm{H}, J=8.1 \mathrm{~Hz}, \mathrm{COCH}_{2}\right), 2.59\left(s, 3 \mathrm{H}, \mathrm{NCCH}_{3}\right), 3.66(t, 2 \mathrm{H}, J=7.0$ $\left.\mathrm{Hz}, \mathrm{NCH}_{2}\right), 6.14\left(\mathrm{~s}, 1 \mathrm{H}, \mathrm{CH}\right.$-vinylic). ${ }^{13} \mathrm{C} \mathrm{NMR}\left(75 \mathrm{MHz}, \mathrm{CDCl}_{3}\right) \delta 16.51\left(\mathrm{CH}_{3}\right), 17.56\left(\mathrm{CH}_{2}\right), 32.58$ $\left(\mathrm{CH}_{3}\right), 33.21\left(\mathrm{CH}_{2}\right), 48.58\left(\mathrm{CH}_{2}\right), 110.52(\mathrm{CH}), 152.03(\mathrm{C}), 175.40(\mathrm{C}), 198.27$ (C). HRMS (ESI) calculated for $\mathrm{C}_{9} \mathrm{H}_{13} \mathrm{NO}_{2} \mathrm{Na}\left([\mathrm{M}+\mathrm{Na}]^{+}\right)$: 190.0838 . Found: 190.0835 .

\section{General Procedure (2) for the Synthesis of the Different Dienes:}

A flame dried $100 \mathrm{ml}$ flask was charged with methyltriphenylphosphonium bromide (1.5-3.0 equiv.) in dry THF at $0{ }^{\circ} \mathrm{C}$ under argon. To the stirred suspension, $n$-BuLi ( 0.95 equiv. $1.6 \mathrm{M}$ solution in hexane) was added dropwise. After complete addition of $n$-BuLi reaction temperature was maintained at $0{ }^{\circ} \mathrm{C}$ for $30 \mathrm{~min}$. and then allowed to warm up to r.t. Stirring was continued for another $1 \mathrm{~h}$. The red solution was then cooled down to $0{ }^{\circ} \mathrm{C}$. A solution of the ketone in dry THF was added dropwise to the stirred red solution. The whole reaction mixture was kept at $0{ }^{\circ} \mathrm{C}$ for another $30 \mathrm{~min}$. Finally the reaction mixture was allowed to warm up to r.t. and was stirred for $12 \mathrm{~h}$. The reaction was quenched with $30 \mathrm{ml}$ of saturated $\mathrm{NH}_{4} \mathrm{Cl}$ solution. The aqueous phase was extracted with $\mathrm{Et}_{2} \mathrm{O}(3 \times 25 \mathrm{ml})$. The combined organic layers were washed with brine $(50 \mathrm{ml})$, dried over $\mathrm{MgSO}_{4}$, and concentrated under reduced pressure. Purification by FC $\left(\mathrm{SiO}_{2}\right)$ (pentane / $t$-butylmethylether) afforded the desired diene. Compunds $\mathbf{7 a}$ and $\mathbf{7 b}$ were prepared according to a literature procedure ${ }^{\mathrm{b}}$. For the synthesis of $\mathbf{7} \mathbf{c}$ see the preparation of compound $8 \mathbf{c}$ below.

$N$-((Z)-1,3-Dimethylbuta-1,3-dienyl)acetamide (7d): Prepared according to the general procedure (2) using methyltriphenylphosphonium bromide $(6.3 \mathrm{~g}, 18 \mathrm{mmol}), n$-BuLi $(10.5 \mathrm{ml}$ of a $1.6 \mathrm{M}$ solution in hexane, $16.8 \mathrm{mmol}), N$-((Z)-1-methyl-3-oxobut-1-enyl)acetamide (1.0 g, $7.1 \mathrm{mmol})$, dry THF (35 ml). Purification by FC (pentane / MTBE 5:1) provided 7d as low melting pale yellow solid (0.46 g, $47 \%)$. IR (neat) 3417br.m, 1651s, 1526m, 1439w, 1375m, 1283m, 1044m, 888w cm ${ }^{-1}$. ${ }^{1} \mathrm{H}$ NMR (400 MHz, DMSO) $\delta 1.84\left(s, 3 \mathrm{H}, \mathrm{CH}_{3}\right), 1.86\left(s, 3 \mathrm{H}, \mathrm{CH}_{3}\right), 1.91\left(s, 3 \mathrm{H}, \mathrm{CH}_{3}\right), 4.84(s, 1 \mathrm{H}, \mathrm{CH}$-vinylic), $4.87(s, 1 \mathrm{H}$, $\mathrm{CH}$-vinylic), $5.52\left(s, 1 \mathrm{H}, \mathrm{CH}\right.$-vinylic), $8.85(s$, br., $1 \mathrm{H}, \mathrm{NH}) .{ }^{13} \mathrm{C}$ NMR (100 MHz, DMSO) $\delta 21.58$ $\left(\mathrm{CH}_{3}\right), 22.39\left(\mathrm{CH}_{3}\right), 22.91\left(\mathrm{CH}_{3}\right), 115.61\left(\mathrm{CH}_{2}\right), 121.98(\mathrm{CH}), 132.42(\mathrm{C}), 140.22(\mathrm{C}), 168.14(\mathrm{C})$. HRMS (ESI) calculated for $\mathrm{C}_{8} \mathrm{H}_{13} \mathrm{NONa}\left([\mathrm{M}+\mathrm{Na}]^{+}\right)$: 162.0889 . Found: 162.0902 .

$N$-((Z)-1,3-Dimethylbuta-1,3-dienyl)benzamide (7e): Prepared according to the general procedure (2) using methyltriphenylphosphonium bromide $(5.2 \mathrm{~g}, 15 \mathrm{mmol}), n$-BuLi $(8.7 \mathrm{ml}$ of a $1.6 \mathrm{M}$ solution in hexane, $14 \mathrm{mmol}), N$-((Z)-1-methyl-3-oxobut-1-enyl)benzamide $(1.0 \mathrm{~g}, 4.9 \mathrm{mmol})$, dry THF (30 ml). 
Purification by FC (pentane / MTBE 10:1) provided 7e as low melting white solid (0.45 g, $45 \%)$. IR (neat) 3250br.m, 2968w, 1663w, 1634s, 1602m, 1578m, 1518s, 1486s, 1433w, 1372m, 1291s, 1185w, $1076 w, 1040 m, 931 m, 889 s, 865 w, 801 \mathrm{~cm}^{-1} .{ }^{1} \mathrm{H}$ NMR (400 MHz, DMSO) $\delta 1.85\left(s, 3 \mathrm{H}, \mathrm{CH}_{3}\right), 2.01$ $\left(s, 3 \mathrm{H}, \mathrm{CH}_{3}\right), 4.85(s, 1 \mathrm{H}, \mathrm{CH}$-vinylic), $4.92(s, 1 \mathrm{H}, \mathrm{CH}$-vinylic), 5.79 ( $s, 1 \mathrm{H}, \mathrm{CH}$-vinylic), 7.56-7.44 ( $m$, $3 \mathrm{H}, \mathrm{CH}$-aromatic), 7.89-7.87 (m, 2H, $\mathrm{CH}$-aromatic), $9.36(\mathrm{~s}$, br., $1 \mathrm{H}, \mathrm{NH}) .{ }^{13} \mathrm{C} \mathrm{NMR}(100 \mathrm{MHz}$, DMSO) $\delta 21.37\left(\mathrm{CH}_{3}\right), 22.62\left(\mathrm{CH}_{3}\right), 116.04\left(\mathrm{CH}_{2}\right), 124.38(\mathrm{CH}), 127.23(\mathrm{CH}), 128.23(\mathrm{CH}), 131.26$ $(\mathrm{CH}), 132.13(\mathrm{C}), 134.14(\mathrm{C}), 140.48$ (C), 164.78 (C). HRMS (ESI) calculated for $\mathrm{C}_{13} \mathrm{H}_{15} \mathrm{NONa}$ $\left([\mathrm{M}+\mathrm{Na}]^{+}\right): 224.1046$. Found: 224.1045 .

$N$-((Z)-1,3-Dimethylbuta-1,3-dienyl)-2,6-difluorobenzamide (7f): Prepared according to the general procedure (2) using methyltriphenylphosphonium bromide $(3.7 \mathrm{~g}, 10 \mathrm{mmol}), n$-BuLi $(5.9 \mathrm{ml}$ of a $1.6 \mathrm{M}$ solution in hexane, $9.5 \mathrm{mmol}), 2$,6-difluoro- $N$-((Z)-1-methyl-3-oxobut-1-enyl)benzamide (1.0 g, 4.1 mmol), dry THF (21 ml). Purification by FC (pentane / MTBE 10:1) provided 7f as a low melting white solid (0.44 g, 44 \%). IR (neat) 3206br.w, 2978w, 2934w, 1646s, 1623s, 1590w, 1518m, 1466s, 1377w, $1304 m, 1269 w, 1237 s, 1195 w, 1007 s, 889 m, 791 s, 761 m \mathrm{~cm}^{-1} .{ }^{1} \mathrm{H}$ NMR (400 MHz, DMSO) $\delta 1.89(s$, $\left.3 \mathrm{H}, \mathrm{CH}_{3}\right), 2.02\left(s, 3 \mathrm{H}, \mathrm{CH}_{3}\right), 4.88(s, 1 \mathrm{H}, \mathrm{CH}$-vinylic), $4.94(s, 1 \mathrm{H}, \mathrm{CH}$-vinylic), $5.73(s, 1 \mathrm{H}, \mathrm{CH}-$ vinylic), 7.19-7.15 (m, 2H, CH-aromatic), 7.55-7.48 (m, 1H, CH-aromatic), 9.75 (s, br., $1 \mathrm{H}, \mathrm{NH}) .{ }^{13} \mathrm{C}$ NMR (100 MHz, MDSO) $\delta 21.18\left(\mathrm{CH}_{3}\right), 22.12\left(\mathrm{CH}_{3}\right), 111.73\left(d d, J_{l}=5.3 \mathrm{~Hz}, J_{2}=19.1 \mathrm{~Hz}, \mathrm{CH}\right)$, $115.41(t, J=23.3 \mathrm{~Hz}, \mathrm{C}), 116.39\left(\mathrm{CH}_{2}\right), 124.29(\mathrm{CH}), 130.74(\mathrm{C}), 131.44(t, J=9.76 \mathrm{~Hz}, \mathrm{CH}), 139.95$ (C), $158.42(\mathrm{C}), 158.66\left(d d, J_{l}=8.1 \mathrm{~Hz}, J_{2}=247.9 \mathrm{~Hz}, \mathrm{C}\right)$. HRMS (ESI) calculated for $\mathrm{C}_{13} \mathrm{H}_{13} \mathrm{~F}_{2} \mathrm{NONa}$ $\left([\mathrm{M}+\mathrm{Na}]^{+}\right): 260.0857$. Found: 260.0855 .

$N$-((Z)-1,3-Dimethylbuta-1,3-dienyl)-4-methoxybenzamide (7g): Prepared according to the general procedure (2) using methyltriphenylphosphonium bromide $(3.8 \mathrm{~g}, 11 \mathrm{mmol}), n$-BuLi $(6.3 \mathrm{ml}$ of a $1.6 \mathrm{M}$ solution in hexane, $10 \mathrm{mmol})$, 4-methoxy- $N$-((Z)-1-methyl-3-oxobut-1-enyl)benzamide (1.0 g, 4.3 mmol), dry THF (22 ml). Purification by FC (pentane / MTBE 6:1) provided $\mathbf{7 g}$ as a low melting white solid (0.4 g, 40 \%). IR (neat) 3287br.m, 2984w, 2944w,1631s, 1603s, 1522m, 1487s, 1459w, 1440m, $1374 m, 1314 m, 1254 s, 1182 s, 1123 w, 1027 s, 929 s, 889 s, 840 s, 766 s \mathrm{~cm}^{-1} .{ }^{1} \mathrm{H}$ NMR (400 MHz, DMSO) $\delta 1.83\left(s, 3 \mathrm{H}, \mathrm{CH}_{3}\right), 2.00\left(s, 3 \mathrm{H}, \mathrm{CH}_{3}\right), 3.80\left(s, 3 \mathrm{H}, \mathrm{OCH}_{3}\right), 4.83(s, 1 \mathrm{H}, \mathrm{CH}$-vinylic), 4.91 (br., $1 \mathrm{H}, \mathrm{CH}$ vinylic), $5.75(s, 1 \mathrm{H}, \mathrm{CH}$-vinylic), $7.00(d, 2 \mathrm{H}, J=8.9 \mathrm{~Hz}, \mathrm{CH}$-aromatic), $7.87(d, 2 \mathrm{H}, J=8.9 \mathrm{~Hz}, \mathrm{CH}$ aromatic), $9.20(s$, br., $1 \mathrm{H}, \mathrm{NH}) .{ }^{13} \mathrm{C}$ NMR (100 MHz, DMSO) $\delta 21.45\left(\mathrm{CH}_{3}\right), 22.75\left(\mathrm{CH}_{3}\right), 55.31$ $\left(\mathrm{CH}_{3}\right), 113.51(\mathrm{CH}), 115.97\left(\mathrm{CH}_{2}\right), 124.08(\mathrm{CH}), 126.37(\mathrm{C}), 129.19(\mathrm{CH}), 132.44(\mathrm{C}), 140.60(\mathrm{C})$, 161.67 (C), $164.36(\mathrm{C})$. HRMS (ESI) calculated for $\mathrm{C}_{14} \mathrm{H}_{17} \mathrm{NO}_{2} \mathrm{Na}\left([\mathrm{M}+\mathrm{Na}]^{+}\right)$: 254.1151 . Found: 254.1143.

\section{General Procedure (3) for the Synthesis of the Hydroamination Reagents:}

A solution of 3-nitro-acrylic acid methyl ester in benzene was added dropwise at $0{ }^{\circ} \mathrm{C}$ under argon to a stirred solution of diene in dry benzene. The reaction temperature was maintained for another $1 \mathrm{~h}$ at $0{ }^{\circ} \mathrm{C}$ then allowed to warm up to r.t. where stirring was continued for $18 \mathrm{~h}$. The reaction mixture was cooled to $0{ }^{\circ} \mathrm{C}$ and DBU was added dropwise and the mixture was stirred for $1 \mathrm{~h}$ at $0{ }^{\circ} \mathrm{C}$. Stirring was continued at r.t. for another $18 \mathrm{~h}$. under argon. Brine was added and the reaction mixture was extracted with $\mathrm{Et}_{2} \mathrm{O}$ $(3 \mathrm{x})$. The combined organic layers were washed with water and finally with brine. The organic phase was dried over $\mathrm{MgSO}_{4}$ and concentrated under reduced pressure. Purification by $\mathrm{FC}\left(\mathrm{SiO}_{2}\right)$ (pentane/tert-butyl-methylether) provided the desired hydroamination reagent.

3-Acetylamino-3,5-dimethylcyclohexa-1,4-dienecarboxylic acid methyl ester (8d): Prepared according to the general procedure (3) using $N$-((Z)-1,3-dimethylbuta-1,3-dienyl)acetamide (7d) $(0.50 \mathrm{~g}$, 
$3.6 \mathrm{mmol})$, 3-nitroacrylic acid methyl ester $(0.71 \mathrm{~g}, 5.4 \mathrm{mmol})$ and DBU (1.0 g, $6.5 \mathrm{mmol})$ in benzene (5 $\mathrm{ml}$ ). Purification by FC (pentane / MTBE 5:1) provided 8d as a pale yellow viscous liquid which was solidified upon standing at low temperature $(0.55 \mathrm{~g}, 69 \%)$. IR (neat) $3475 \mathrm{br} . \mathrm{w}, 2970 \mathrm{~m}, 1719 \mathrm{~s}, 1683 \mathrm{~s}$, $1438 m, 1301 s, 1259 s, 1177 m, 1073 m, 997 w, 934 m, 852 m, 745 s \mathrm{~cm}^{-1} .{ }^{1} \mathrm{H}$ NMR $\left(300 \mathrm{MHz}, \mathrm{C}_{6} \mathrm{D}_{6}\right) \delta 1.37$ $\left(s, 3 \mathrm{H}, \mathrm{CH}_{3}\right), 1.53\left(s, 3 \mathrm{H}, \mathrm{CH}_{3}\right), 1.54\left(s, 3 \mathrm{H}, \mathrm{CH}_{3}\right), 2.93-2.63\left(m, 2 \mathrm{H}, \mathrm{CH}_{2}\right), 3.41\left(s, 3 \mathrm{H}, \mathrm{CO}_{2} \mathrm{CH}_{3}\right), 5.30$ $\left(s, 1 \mathrm{H}, \mathrm{CH}\right.$-vinylic), $5.36(s, \mathrm{br} ., 1 \mathrm{H}, \mathrm{NH}), 7.27\left(s\right.$, br., $1 \mathrm{H}, \mathrm{CH}$-vinylic). ${ }^{13} \mathrm{C} \mathrm{NMR}\left(75 \mathrm{MHz}, \mathrm{C}_{6} \mathrm{D}_{6}\right) \delta$ $22.55\left(\mathrm{CH}_{3}\right), 23.64\left(\mathrm{CH}_{3}\right), 27.88\left(\mathrm{CH}_{3}\right), 30.99\left(\mathrm{CH}_{2}\right), 51.29\left(\mathrm{CH}_{3}\right), 53.22(\mathrm{C}), 124.54(\mathrm{CH}), 132.47(\mathrm{C})$, $139.96(\mathrm{CH}), 167.08(\mathrm{C}), 168.75(\mathrm{C})$. HRMS (ESI) calculated for $\mathrm{C}_{12} \mathrm{H}_{17} \mathrm{NO}_{3} \mathrm{Na}\left([\mathrm{M}+\mathrm{Na}]^{+}\right): 246.1101$. Found: 246.1091.

3-Benzoylamino-3,5-dimethylcyclohexa-1,4-dienecarboxylic acid methyl ester (8e): Prepared according to the general procedure (3) using $N$-((Z)-1,3-Dimethylbuta-1,3-dienyl)benzamide (7e) (2.50 $\mathrm{g}, 12.4 \mathrm{mmol})$, 3-nitro-acrylic acid methyl ester $(2.7 \mathrm{~g}, 21 \mathrm{mmol})$ and DBU $(4.1 \mathrm{~g}, 27 \mathrm{mmol})$ in benzene $(20 \mathrm{ml}$ ). Purification by FC (pentane / MTBE 5:1) provided 8e as a pale yellow viscous liquid which was solidified upon standing at low temperature (2.3 g, $66 \%$ ). IR (neat) 3235br.m, 3056w, 2948w, $1717 s, 1688 w, 1636 s, 1531 s, 1493 w, 1441 m, 1350 w, 1312 s, 1290 s, 1256 s, 1191 s, 1143 w, 1087 w$, $1068 s, 987 w, 927 m, 831 m, 748 m \mathrm{~cm}^{-1} .{ }^{1} \mathrm{H}$ NMR (400 MHz, $\left.\mathrm{C}_{6} \mathrm{D}_{6}\right) \delta 1.49\left(s, 3 \mathrm{H}, \mathrm{CH}_{3}\right), 1.52(s, 3 \mathrm{H}$, $\left.\mathrm{CH}_{3}\right), 2.95-2.67\left(m, 2 \mathrm{H}, \mathrm{CH}_{2}\right), 3.39\left(s, 3 \mathrm{H}, \mathrm{CO}_{2} \mathrm{CH}_{3}\right), 5.41-5.4(m, 1 \mathrm{H}, \mathrm{CH}$-vinylic), 5.88 ( $s$, br., $1 \mathrm{H}$, $\mathrm{NH}$ ), 7.09-6.98 ( $m, 3 \mathrm{H}, \mathrm{CH}$-aromatic), 7.42-7.41 ( $m, 1 \mathrm{H}, \mathrm{CH}$-vinylic), 7.63-7.60 ( $m, 2 \mathrm{H}, \mathrm{CH}$-aromatic). ${ }^{13} \mathrm{C}$ NMR $\left(100 \mathrm{MHz}, \mathrm{C}_{6} \mathrm{D}_{6}\right) \delta 22.60\left(\mathrm{CH}_{3}\right), 27.93\left(\mathrm{CH}_{3}\right), 31.08\left(\mathrm{CH}_{2}\right), 51.27\left(\mathrm{CH}_{3}\right), 53.62(\mathrm{C}), 124.35$ $(\mathrm{CH}), 127.34(\mathrm{CH}), 128.46(\mathrm{CH}), 131.08(\mathrm{CH}), 132.99(\mathrm{C}), 135.90(\mathrm{CH}), 139.66(\mathrm{C}), 166.04(\mathrm{C})$, 167.02 (C). HRMS (ESI) calculated for $\mathrm{C}_{17} \mathrm{H}_{19} \mathrm{NO}_{3} \mathrm{Na}\left([\mathrm{M}+\mathrm{Na}]^{+}\right)$: 308.1257. Found: 308.1255.

3-(2,6-Difluorobenzoylamino)-3,5-dimethylcyclohexa-1,4-dienecarboxylic acid methyl ester (8f): Prepared according to the general procedure (3) (for the Diels-Alder reaction, the reaction mixture was heated at $60{ }^{\circ} \mathrm{C}$ for $6 \mathrm{~h}$ and complete consumption of the diene was confirmed by TLC) using $N$-((Z)1,3-dimethylbuta-1,3-dienyl)-2,6-difluorobenzamide (7f) $(0.80 \mathrm{~g}, 3.4 \mathrm{mmol})$, 3-nitro-acrylic acid methyl ester $(0.65 \mathrm{~g}, 4.9 \mathrm{mmol})$, and DBU $(1.1 \mathrm{~g}, 7.2 \mathrm{mmol})$ in benzene $(10 \mathrm{ml})$. Purification by FC (pentane / MTBE 3:1) provided 8f as a colourless oil (0.68 g, $63 \%)$. IR (neat) 3285br.m, 2953w, 1718s, 1658s, $1624 s, 1590 m, 1532 s, 1567 s, 1259 s, 1195 w, 1072 m, 1008 s, 793 s, 744 m \mathrm{~cm}^{-1} .{ }^{1} \mathrm{H}$ NMR (400 MHz, $\left.\mathrm{C}_{6} \mathrm{D}_{6}\right) \delta 1.42\left(s, 3 \mathrm{H}, \mathrm{CH}_{3}\right), 1.48\left(s, 3 \mathrm{H}, \mathrm{CH}_{3}\right), 2.90-2.61\left(\mathrm{~m}, 2 \mathrm{H}, \mathrm{CH}_{2}\right), 3.35\left(\mathrm{~s}, 3 \mathrm{H}, \mathrm{CO}_{2} \mathrm{CH}_{3}\right), 5.38-5.37$ ( $m, 1 \mathrm{H}, \mathrm{CH}$-vinylic), 5.61 ( $\mathrm{s}$, br., 1H, $\mathrm{NH}$ ), 6.40-6.34 (m, 2H, $\mathrm{CH}$-aromatic), 6.61-6.53 ( $m, 1 \mathrm{H}, \mathrm{CH}$ aromatic), 7.32-7.31 (m, 1H, CH-vinylic). $\left.{ }^{13} \mathrm{C} \mathrm{NMR} \mathrm{(100} \mathrm{MHz,} \mathrm{C}_{6} \mathrm{D}_{6}\right) \delta 22.50\left(\mathrm{CH}_{3}\right), 27.95\left(\mathrm{CH}_{3}\right)$, $30.97\left(\mathrm{CH}_{2}\right), 51.27\left(\mathrm{CH}_{3}\right), 54.36(\mathrm{C}), 111.84-111.59(m, \mathrm{CH}), 116.11(t, J=21.8 \mathrm{~Hz}, \mathrm{C}), 123.85(\mathrm{CH})$, $128.62(\mathrm{C}), 131.01(t, J=9.9 \mathrm{~Hz}, \mathrm{CH}), 133.23(\mathrm{C}), 139.0(\mathrm{CH}), 160.12\left(d d, J_{l}=7.0 \mathrm{~Hz}, J_{2}=250.4 \mathrm{~Hz}\right.$, C), 166.9 (C). HRMS (ESI) calculated for $\mathrm{C}_{17} \mathrm{H}_{17} \mathrm{~F}_{2} \mathrm{NO}_{3} \mathrm{Na}\left([\mathrm{M}+\mathrm{Na}]^{+}\right)$: 344.1069. Found: 344.1077.

3-(4-Methoxybenzoylamino)-3,5-dimethylcyclohexa-1,4-dienecarboxylic acid methyl ester (8g): Prepared according to the general procedure (3) using $N$-((Z)-1,3-dimethylbuta-1,3-dienyl)-4methoxybenzamide $(\mathbf{7 g})(1.0 \mathrm{~g}, 4.3 \mathrm{mmol})$, 3-nitro-acrylic acid methyl ester $(0.85 \mathrm{~g}, 6.5 \mathrm{mmol})$, and DBU (1.4 g, $9.5 \mathrm{mmol})$ in benzene $(10 \mathrm{ml})$. Purification by FC (pentane / MTBE 5:1) provided $8 \mathrm{~g}$ as a white solid (0.54 g, 40 \%). IR (neat) 3276br.m, 2988w, 2947w, 1709s, 1692m, 1632s, 1606s, 1534m, $1503 s, 1440 s, 1350 m, 1315 m, 1257 s, 1179 s, 1128 m, 1093 m, 1068 m, 1031 s, 927 m, 846 s, 771 m, 747 s$ $\mathrm{cm}^{-1} .{ }^{1} \mathrm{H}$ NMR $\left(400 \mathrm{MHz}, \mathrm{C}_{6} \mathrm{D}_{6}\right) \delta 1.52\left(s, 6 \mathrm{H}, \mathrm{CH}_{3}\right), 2.99-2.69\left(m, 2 \mathrm{H}, \mathrm{CH}_{2}\right), 3.18\left(\mathrm{~s}, 3 \mathrm{H}, \mathrm{OCH}_{3}\right), 3.39$ $\left(s, 3 \mathrm{H}, \mathrm{CO}_{2} \mathrm{CH}_{3}\right), 5.42(s, 1 \mathrm{H}, \mathrm{CH}$-vinylic), $5.62(s$, br., $1 \mathrm{H}, \mathrm{NH}), 6.60(d, 2 \mathrm{H}, J=8.8 \mathrm{~Hz}, \mathrm{CH}$-aromatic), 7.49-7.48 ( $m, 1 \mathrm{H}, \mathrm{CH}$-vinylic), $7.59\left(d, 2 \mathrm{H}, J=8.8 \mathrm{~Hz}, \mathrm{CH}\right.$-aromatic). ${ }^{13} \mathrm{C} \mathrm{NMR}\left(100 \mathrm{MHz}, \mathrm{C}_{6} \mathrm{D}_{6}\right) \delta$ $22.61\left(\mathrm{CH}_{3}\right), 27.96\left(\mathrm{CH}_{3}\right), 31.13\left(\mathrm{CH}_{2}\right), 51.24\left(\mathrm{CH}_{3}\right), 53.53(\mathrm{C}), 54.80\left(\mathrm{CH}_{3}\right), 113.81(\mathrm{CH}), 124.52$ $(\mathrm{CH}), 129.12(\mathrm{CH}), 132.98(\mathrm{C}), 139.79(\mathrm{CH}), 162.31(\mathrm{C}), 165.49(\mathrm{C}), 167.03(\mathrm{C})$. HRMS (ESI) calculated for $\mathrm{C}_{18} \mathrm{H}_{21} \mathrm{NO}_{4} \mathrm{Na}\left([\mathrm{M}+\mathrm{Na}]^{+}\right)$: 338.1363 . Found: 338.1361 .

3,5-Dimethyl-3-(2-oxopyrrolidin-1-yl)cyclohexa-1,4-dienecarboxylic acid methyl ester (8h): Prepared using 1-((Z)-1,3-Dimethylbuta-1,3-dienyl)pyrrolidin-2-one, which was prepared according to 
the general procedure (2) using methyltriphenylphosphonium bromide (3.2 g, $8.9 \mathrm{mmol}), n$-BuLi (5.2 ml of a 1.6 M solution in hexane, $8.4 \mathrm{mmol}), 1-((Z)-1-M e t h y l-3$-oxobut-1-enyl)pyrrolidin-2-one (1.0 g, 6.0 mmol), dry THF (20 ml). Purification by FC (pentane / MTBE 1:2) provided 1-((Z)-1,3-Dimethylbuta1,3-dienyl)pyrrolidin-2-one as colourless oil (0.54 g, 55\%). IR (neat) 3368br.m, 2978m, 1682s, 1461w, $1414 m, 1291 s, 1073 w, 994 m, 840 w \mathrm{~cm}^{-1} .{ }^{1} \mathrm{H}$ NMR $\left(400 \mathrm{MHz}, \mathrm{C}_{6} \mathrm{D}_{6}\right) \delta 1.23-1.13\left(m, 2 \mathrm{H}, \mathrm{CH}_{2}\right), 1.75(s$, $\left.3 \mathrm{H}, \mathrm{CH}_{3}\right), 2.01-1.97\left(m, 2 \mathrm{H}, \mathrm{COCH}_{2}\right), 2.37-2.35\left(m, 3 \mathrm{H}, \mathrm{CH}_{3}\right), 2.84-2.79\left(m, 2 \mathrm{H}, \mathrm{NCH}_{2}\right), 4.87(s, 1 \mathrm{H}$, $\mathrm{CH}$-vinylic), $4.99\left(\mathrm{~s}, 1 \mathrm{H}, \mathrm{CH}\right.$-vinylic), $5.48\left(\mathrm{~s}, 1 \mathrm{H}, \mathrm{CH}\right.$-vinylic). ${ }^{13} \mathrm{C} \mathrm{NMR}\left(100 \mathrm{MHz}, \mathrm{C}_{6} \mathrm{D}_{6}\right) \delta 16.95$ $\left(\mathrm{CH}_{3}\right), 17.99\left(\mathrm{CH}_{2}\right), 24.26\left(\mathrm{CH}_{3}\right), 32.65\left(\mathrm{CH}_{2}\right), 48.61\left(\mathrm{CH}_{2}\right), 114.87\left(\mathrm{CH}_{2}\right), 118.00(\mathrm{CH}), 136.99(\mathrm{C})$, 140.74 (C), 173.04 (C). HRMS (ESI) calculated for $\mathrm{C}_{10} \mathrm{H}_{15} \mathrm{NONa}\left([\mathrm{M}+\mathrm{Na}]^{+}\right)$: 188.1046 . Found: 188.1037 .

1-((Z)-1,3-Dimethylbuta-1,3-dienyl)pyrrolidin-2-one (1.1 g, $6.6 \mathrm{mmol}), 3$-nitroacrylic acid methyl ester $(1.3 \mathrm{~g}, 9.9 \mathrm{mmol})$, and DBU $(2.2 \mathrm{~g}, 14 \mathrm{mmol})$ in benzene $(10 \mathrm{ml})$ according to the general procedure $(3)$. Purification by FC (pentane / MTBE 1:2) provided $\mathbf{8 h}$ as a colourless oil (1.1 g, $67 \%$ ). IR (neat) 3288br.m, 2970w, 1720s, 1658s, 1538s, 1438s, 1372s, 1258s, 1195m, 1127w, 1071m, 932w, 849w, $752 \mathrm{~cm}^{-1} .{ }^{1} \mathrm{H}$ NMR $\left(300 \mathrm{MHz}, \mathrm{C}_{6} \mathrm{D}_{6}\right) \delta 1.21-1.11\left(m, 2 \mathrm{H}, \mathrm{CH}_{2}\right), 1.48-1.47\left(m, 3 \mathrm{H}, \mathrm{NCCH}_{3}\right), 1.75(s$, $3 \mathrm{H}, \mathrm{CH}_{3}$-vinylic), 1.98-1.92 ( $\left.\mathrm{m}, 2 \mathrm{H}, \mathrm{COCH}_{2}\right), 2.83-2.60\left(\mathrm{~m}, 4 \mathrm{H}, \mathrm{NCH}_{2}\right.$ and $\mathrm{CH}_{2}$-allylic), $3.39(\mathrm{~s}, 3 \mathrm{H}$, $\left.\mathrm{CO}_{2} \mathrm{CH}_{3}\right), 5.43-5.40\left(\mathrm{~m}, 1 \mathrm{H}, \mathrm{CH}\right.$-vinylic), 7.30-7.28 ( $m, 1 \mathrm{H}, \mathrm{CH}$-vinylic). ${ }^{13} \mathrm{C} \mathrm{NMR}\left(75 \mathrm{MHz}, \mathrm{C}_{6} \mathrm{D}_{6}\right) \delta$ $18.10\left(\mathrm{CH}_{2}\right), 22.53\left(\mathrm{CH}_{3}\right), 26.86\left(\mathrm{CH}_{3}\right), 30.72\left(\mathrm{CH}_{2}\right), 32.73\left(\mathrm{CH}_{2}\right), 45.90\left(\mathrm{CH}_{2}\right), 51.31\left(\mathrm{CH}_{3}\right), 56.34(\mathrm{C})$, $123.61(\mathrm{CH}), 132.12(\mathrm{C}), 139.04(\mathrm{CH}), 166.88(\mathrm{C}), 174.47(\mathrm{C})$. HRMS (ESI) calculated for $\mathrm{C}_{14} \mathrm{H}_{19} \mathrm{NO}_{3} \mathrm{Na}\left([\mathrm{M}+\mathrm{Na}]^{+}\right): 272.1257$. Found 272.1250.

3-(3-tert-Butylureido)-3,5-dimethylcyclohexa-1,4-dienecarboxylic acid methyl ester (8c): To a solution of 2,4-dimethylpenta-2,4-dienoic acid (0.5 g, $4 \mathrm{mmol})$ in acetone (10 ml) $\mathrm{Et}_{3} \mathrm{~N}$ (0.90 ml, 6.35 mmol) was added. After cooling to $0{ }^{\circ} \mathrm{C}$ a solution of ethyl chloroformate $(0.64 \mathrm{~g}, 6.0 \mathrm{mmol})$ in acetone $(5 \mathrm{~mL})$ was added dropwise. The mixture was stirred for $30 \mathrm{~min}$ at $0{ }^{\circ} \mathrm{C}$ and a solution of $\mathrm{NaN}_{3}(0.36 \mathrm{~g}$, $5.6 \mathrm{mmol})$ in water $(2 \mathrm{ml})$ was added slowly $\left(0{ }^{\circ} \mathrm{C}\right)$. After stirring for $2 \mathrm{~h}$ at $0{ }^{\circ} \mathrm{C}$ the reaction mixture was poured onto ice-water and extracted with $\mathrm{CH}_{2} \mathrm{Cl}_{2}(3 \mathrm{x})$. The combined organic layers were dried over $\mathrm{MgSO}_{4}$ and $90 \%$ of the solvent was removed under reduced pressure. Purification by FC (pentene / MTBE 15:1) provided the desired azide ${ }^{\mathrm{b}}$.

A solution of the azide in dry toluene $(10 \mathrm{ml})$ was heated under reflux for $3 \mathrm{~h}$ under argon. The solution was cooled to $0{ }^{\circ} \mathrm{C}$ and a solution of tert-butylamine $(0.19 \mathrm{~g}, 2.7 \mathrm{mmol})$ in dry toluene $(5 \mathrm{ml})$ was added dropwise. The mixture was heated to reflux for $1 \mathrm{~h}$ and then allowed to stir at r.t. over night. A white solid powder was precipitated. The solvent was removed under reduced pressure at r.t. and the solid was used directly for the next step without purification.

To a stirred suspension of the 1-tert-Butyl-3-((Z)-1,3-dimethylbuta-1,3-dienyl)urea (7c) (0.4 g, $2 \mathrm{mmol})$ in benzene $(15 \mathrm{ml})$ a solution of 3-nitro-acrylic acid methyl ester $(0.4 \mathrm{~g}, 3 \mathrm{mmol})$ in benzene was added dropwise at $0{ }^{\circ} \mathrm{C}$ under argon. The reaction mixture was kept at $0{ }^{\circ} \mathrm{C}$ for $1 \mathrm{~h}$ and was then allowed to warm to r.t. After stirring at r.t. for $12 \mathrm{~h}$ the solution was cooled at $0{ }^{\circ} \mathrm{C}$ and DBU $(0.46 \mathrm{~g}, 3.0 \mathrm{mmol})$ was added dropwise. The reaction temperature was maintained for another $1 \mathrm{~h}$ at $0{ }^{\circ} \mathrm{C}$ and then allowed to warm up to r.t where stirring was continued for $18 \mathrm{~h}$. Brine was added and the reaction mixture was extracted with $\mathrm{Et}_{2} \mathrm{O}(3 \mathrm{x})$. The combined organic layers were washed with water, dried over $\mathrm{MgSO}_{4}$, concentrated under reduced pressure. Purification by FC (pentane / MTBE 1:2) provided 8c as a white solid powder $(0.38 \mathrm{~g}, 66 \%$ ). IR (neat) 3344br.m, 2967w, 2933w, 1712s, 1687w, 1635s, 1553s, 1449m, $1437 m, 1360 w, 1258 s, 1255 s, 1220 m, 1193 m, 1141 s, 1074 s, 1063 m, 986 w, 929 m, 834 w, 777 w, 742 s$ $\mathrm{cm}^{-1} .{ }^{1} \mathrm{H}$ NMR $(300 \mathrm{MHz}, \mathrm{DMSO}) \delta 1.15\left(s, 9 \mathrm{H}, \mathrm{C}\left(\mathrm{CH}_{3}\right)_{3}\right), 1.26\left(s, 3 \mathrm{H} . \mathrm{CH}_{3}\right), 1.71\left(\mathrm{~s}, 3 \mathrm{H}, \mathrm{CH}_{3^{-}}\right.$ vinylic), 2.75-2.57 ( $\left.m, 2 \mathrm{H}, \mathrm{CH}_{2}\right), 3.68\left(s, 3 \mathrm{H}, \mathrm{CO}_{2} \mathrm{CH}_{3}\right), 5.31(s, 1 \mathrm{H}, \mathrm{CH}$-vinylic), $5.46(s, 1 \mathrm{H}, \mathrm{NH})$, $5.79(s, 1 \mathrm{H}, \mathrm{NH}), 6.91-6.90\left(\mathrm{~m}, 1 \mathrm{H}, \mathrm{CH}\right.$-vinylic). ${ }^{13} \mathrm{C} \mathrm{NMR}(75 \mathrm{MHz}, \mathrm{DMSO}) \delta 22.26\left(\mathrm{CH}_{3}\right), 28.39$ $\left(\mathrm{CH}_{3}\right), 29.24\left(\mathrm{CH}_{3}\right), 29.96\left(\mathrm{CH}_{2}\right), 48.92(\mathrm{C}), 51.12(\mathrm{C}), 51.59\left(\mathrm{CH}_{3}\right), 125.12(\mathrm{CH}), 125.76(\mathrm{C}), 130.14$ 
(C), $142.18(\mathrm{CH}), 156.26(\mathrm{C}), 166.66(\mathrm{C})$. HRMS (ESI) calculated for $\mathrm{C}_{15} \mathrm{H}_{24} \mathrm{~N}_{2} \mathrm{O}_{3} \mathrm{Na}\left([\mathrm{M}+\mathrm{Na}]^{+}\right)$: 303.1679. Found: 303.1672.

\section{General Procedure (4) for the Hydroamination of Norbornene with Different Reagents and for the Hydroamination of Different Olefins with Reagent 8a:}

A mixture of the hydroamination reagent (1 equiv.), the olefin (2.5 to 6.0 equiv.), di-tert-butyl-peroxide (DTBP) ( 0.5 equiv.) and thiophenol ( 0.15 to 0.20 equiv.) in dry benzene was placed in a sealed tube under argon. The reaction mixture was heated in the sealed tube under argon at $140{ }^{\circ} \mathrm{C}$ (oil bath temperature) for $18 \mathrm{~h}$. The reaction mixture was allowed to cool to room temperature and the solvent was removed under reduced pressure. Purification by $\mathrm{FC}\left(\mathrm{SiO}_{2}\right)$ afforded the desired hydroamination product.

Octylcarbamic acid methyl ester (9a): Prepared according to the general procedure (4) using reagent 8a $(0.10 \mathrm{~g}, 0.41 \mathrm{mmol}), 1$-octene $(0.18 \mathrm{~g}, 1.6 \mathrm{mmol})$, DTBP $(30 \mathrm{mg}, 0.20 \mathrm{mmol})$ and thiophenol $(7.0$ $\mathrm{mg}, 0.06 \mathrm{mmol}$ ) in dry benzene $(1 \mathrm{ml})$. Purification by FC (pentane / tert-butylmethylether 6:1) provided 9a as a low melting white solid with another regio-isomer in 1:7 ratio (ratio was determined by ${ }^{1} \mathrm{H}$ NMR spectroscopy) (40 mg, $51 \%$ (combined yield of both isomers)). Physical data of the major isomer is given. IR (neat) 3335br.m, 2927s, 2856m, 1703s, 1538s, 1465m, 1262s, 1146w, 776 $\mathrm{cm}^{-1}$. ${ }^{1} \mathrm{H}$ NMR $\left(400 \mathrm{MHz}, \mathrm{CDCl}_{3}\right) \delta 0.87\left(t, 3 \mathrm{H}, J=6.8 \mathrm{~Hz}, \mathrm{CH}_{3}\right), 1.30-1.26\left(m, 10 \mathrm{H}, \mathrm{CH}_{2}\right), 1.49-1.44(m, 2 \mathrm{H}$, $\left.\mathrm{NHCH}_{2} \mathrm{CH}_{2}\right), 3.15-3.14\left(m, 2 \mathrm{H}, \mathrm{NHCH}_{2}\right), 3.65\left(s, 3 \mathrm{H}, \mathrm{CO}_{2} \mathrm{CH}_{3}\right), 4.65(s$, br., $1 \mathrm{H}, \mathrm{NH}) .{ }^{13} \mathrm{C} \mathrm{NMR}(100$ $\left.\mathrm{MHz}, \mathrm{CDCl}_{3}\right) \delta 14.02\left(\mathrm{CH}_{3}\right), 22.60\left(\mathrm{CH}_{2}\right), 26.71\left(\mathrm{CH}_{2}\right), 29.17\left(\mathrm{CH}_{2}\right), 29.21\left(\mathrm{CH}_{2}\right), 30.01\left(\mathrm{CH}_{2}\right), 31.76$ $\left(\mathrm{CH}_{2}\right), 41.14\left(\mathrm{CH}_{2}\right), 51.94\left(\mathrm{CH}_{3}\right), 157.06(\mathrm{C})$. HRMS (ESI) calculated for $\mathrm{C}_{10} \mathrm{H}_{21} \mathrm{NO}_{2} \mathrm{Na}\left([\mathrm{M}+\mathrm{Na}]^{+}\right)$: 210.1465. Found: 210.1466.

Bicyclo[2.2.1]hept-2-yl-carbamic acid methyl ester (10a): Prepared according to the general procedure (4) using reagent $8 \mathbf{a}(0.10 \mathrm{~g}, 0.41 \mathrm{mmol})$, norbornene $(98 \mathrm{mg}, 1.0 \mathrm{mmol})$, DTBP (30 mg, 0.20 $\mathrm{mmol})$ and thiophenol $(7.0 \mathrm{mg}, 0.06 \mathrm{mmol})$ in dry benzene $(1 \mathrm{ml})$. Purification by FC (pentane / tertbutylmethylether 10:1) provided 10a as a white solid ${ }^{\mathrm{b}}(40 \mathrm{mg}, 57 \%$ ). IR (neat) 3322br. $\mathrm{m}, 2952 \mathrm{~s}$, $2872 m, 1689 s, 1535 s, 1451 m, 1365 w, 1304 m, 1252 s, 1190 m, 1071 s, 1009 m, 779 m, 631 s \mathrm{~cm}^{-1}$. ${ }^{1} \mathrm{H}$ NMR (300 MHz, $\left.\mathrm{CDCl}_{3}\right) \delta$ 1.29-1.03 ( $\left.m, 5 \mathrm{H}, \mathrm{CH}_{3}\right), 1.53-1.35\left(m, 2 \mathrm{H}, \mathrm{CH}_{2}\right), 1.77-1.69(\mathrm{~m}, 1 \mathrm{H}, \mathrm{CH} 2), 2.17(s$, br., $1 \mathrm{H}, \mathrm{CH}), 2.21(\mathrm{~s}$, br., $1 \mathrm{H}, \mathrm{CH}), 3.46(s, b r, 1 \mathrm{H}, \mathrm{NHCH}), 3.61(\mathrm{~s}, 3 \mathrm{H}, \mathrm{CH} 3), 4.54(s, b r, 1 \mathrm{H}, \mathrm{NH}) .{ }^{13} \mathrm{C}$ NMR $\left(75 \mathrm{MHz}, \mathrm{CDCl}_{3}\right) \delta 26.29\left(\mathrm{CH}_{2}\right), 28.09\left(\mathrm{CH}_{2}\right), 35.20\left(\mathrm{CH}_{2}\right), 35.61(\mathrm{CH}), 42.51\left(\mathrm{CH}_{2}\right), 51.79$ $(\mathrm{CH}), 54.31\left(\mathrm{CH}_{3}\right), 156.09(\mathrm{C})$. HRMS (ESI) calculated for $\mathrm{C}_{9} \mathrm{H}_{15} \mathrm{NO}_{2} \mathrm{Na}\left([\mathrm{M}+\mathrm{Na}]^{+}\right): 192.0995$. Found: 192.0997.

Bicyclo[2.2.1]hept-2-yl-carbamic acid tert-butyl ester (10b): Prepared according to the general procedure (4) using reagent $\mathbf{8 b}(0.10 \mathrm{~g}, 0.35 \mathrm{mmol})$, norbornene $(84 \mathrm{mg}, 0.90 \mathrm{mmol})$, DTBP $(26 \mathrm{mg}$, $0.17 \mathrm{mmol})$ and thiophenol $(6.0 \mathrm{mg}, 0.05 \mathrm{mmol})$ in dry benzene $(1 \mathrm{ml})$. Purification by FC (pentane / tert-butylmethylether 10:1) provided 10b as a white solid ${ }^{\mathrm{b}}$ (42 mg, $56 \%$ ). IR (neat) 3352br. $\mathrm{m}, 2955 \mathrm{~m}$, $2874 w, 1680 s, 1519 s, 1452 w, 1365 m, 1248 s, 1162 s, 1066 s, 997 m, 780 w \mathrm{~cm}^{-1} .{ }^{1} \mathrm{H}$ NMR (300 MHz, $\left.\mathrm{CDCl}_{3}\right) \delta 1.41\left(s, 9 \mathrm{H}, \mathrm{CH}_{3}\right) .1 .54-1.03\left(m, 7 \mathrm{H}, \mathrm{CH}_{2}\right), 1.76-1.68\left(m, 1 \mathrm{H}, \mathrm{CH}_{2}\right), 2.16(s$, br., $1 \mathrm{H}, \mathrm{CH}), 2.21$ $(s$, br., $1 \mathrm{H}, \mathrm{CH}), 3.41(s$, br., $1 \mathrm{H}, \mathrm{NHCH}), 4.33(s$, br., $1 \mathrm{H}, \mathrm{NH}) .{ }^{13} \mathrm{C} \mathrm{NMR}\left(75 \mathrm{MHz}, \mathrm{CDCl}_{3}\right) \delta 26.39$ $\left(\mathrm{CH}_{2}\right), 28.15\left(\mathrm{CH}_{2}\right), 28.46\left(\mathrm{CH}_{3}\right), 35.25\left(\mathrm{CH}_{2}\right), 35.61(\mathrm{CH}), 40.48\left(\mathrm{CH}_{2}\right), 42.56(\mathrm{CH}), 54.05(\mathrm{CH}), 79.02$ (C), 155.23 (C). HRMS (ESI) calculated for $\mathrm{C}_{12} \mathrm{H}_{21} \mathrm{NO}_{2} \mathrm{Na}\left([\mathrm{M}+\mathrm{Na}]^{+}\right)$: 234.1470. Found: 234.1465.

1-Bicyclo[2.2.1]hept-2-yl-3-tert-butyl-urea (10c): Prepared according to the general procedure (4) using reagent $8 \mathbf{c}(0.10 \mathrm{~g}, 0.36 \mathrm{mmol})$, norbornene $(84 \mathrm{mg}, 0.90 \mathrm{mmol})$, DTBP $(26 \mathrm{mg}, 0.17 \mathrm{mmol})$ and thiophenol $(6.0 \mathrm{mg}, 0.05 \mathrm{mmol})$ in dry benzene $(1 \mathrm{ml})$. Purification by FC (pentane / tert- 
butylmethylether 3:1) provided 10c as a white solid (6 mg, $8 \%$ ). IR (neat) 3323br.m, 2954s, 2870m, $1624 s, 1558 s, 1475 w, 1450 m, 1389 m, 1360 m, 1306 m, 1278 m, 1216 s, 1181 w, 1136 m, 837 w, 775 w \mathrm{~cm}^{-1}$. ${ }^{1} \mathrm{H}$ NMR $\left(300 \mathrm{MHz}, \mathrm{CDCl}_{3}\right) \delta 1.20-1.00\left(m, 4 \mathrm{H}, \mathrm{CH}_{2}\right), 1.27\left(s, 9 \mathrm{H}, \mathrm{C}\left(\mathrm{CH}_{3}\right)_{3}\right), 1.48-1.32(m, 3 \mathrm{H}, \mathrm{CH} H$, $\mathrm{CH}_{2}$ ), 1.70-1.63 ( $\left.m, 1 \mathrm{H}, \mathrm{CHH}\right), 2.10(s$, br., $1 \mathrm{H}, \mathrm{CH}), 2.17$ ( $s$, br., $\left.1 \mathrm{H}, \mathrm{CH}\right), 3.43-3.40(\mathrm{~m}, 1 \mathrm{H}, \mathrm{NHCH})$, $4.95(s$, br., $2 \mathrm{H}, \mathrm{NH}) .{ }^{13} \mathrm{C}$ NMR $\left(75 \mathrm{MHz}, \mathrm{CDCl}_{3}\right) \delta 26.43\left(\mathrm{CH}_{2}\right), 28.17\left(\mathrm{CH}_{2}\right), 29.53\left(\mathrm{CH}_{3}\right), 35.20$ $\left(\mathrm{CH}_{2}\right), 35.54(\mathrm{CH}), 40.56\left(\mathrm{CH}_{2}\right), 42.72(\mathrm{CH}), 49.95(\mathrm{C}), 53.20(\mathrm{CH}), 157.55(\mathrm{C})$. HRMS (ESI) calculated for $\mathrm{C}_{12} \mathrm{H}_{22} \mathrm{~N}_{2} \mathrm{ONa}\left([\mathrm{M}+\mathrm{Na}]^{+}\right)$: 233.1624. Found: 233.1622 .

$N$-Bicyclo[2.2.1]hept-2-yl-acetamide (10d): Prepared according to the general procedure (4) using reagent 8d $(0.10 \mathrm{~g}, 0.45 \mathrm{mmol})$, norbornene $(0.10 \mathrm{~g}, 1.1 \mathrm{mmol})$, DTBP $(32 \mathrm{mg}, 0.22 \mathrm{mmol})$ and thiophenol $(7.4 \mathrm{mg}, 0.06 \mathrm{mmol})$ in dry benzene $(1 \mathrm{ml})$. Purification by FC (pentane / tertbutylmethylether 10:1) provided 10d as a white solid ${ }^{\mathrm{c}}(28 \mathrm{mg}, 41 \%$ ). IR (neat) 3274br.m, 3078w, $2955 s, 2870 m, 1638 s, 1550 s, 1478 w, 1440 m, 1373 m, 1311 m, 1290 m, 1159 w, 1112 w, 1039 w, 728 m \mathrm{~cm}^{-}$ ${ }^{1} .{ }^{1} \mathrm{H}$ NMR $\left(400 \mathrm{MHz}, \mathrm{CDCl}_{3}\right) \delta$ 1.32-1.07 (m, 5H, $\left.\mathrm{CH}_{2}, \mathrm{CHH}\right), 1.53-1.39\left(m, 2 \mathrm{H}, \mathrm{CH}_{2}\right), 1.81-1.75(m$, $1 \mathrm{H}, \mathrm{CH} H), 1.94\left(s, 3 \mathrm{H}, \mathrm{COCH}_{3}\right), 2.19-2.18$ (br., $\left.1 \mathrm{H}, \mathrm{CH}\right), 2.26(s$, br., $1 \mathrm{H}, \mathrm{CH}), 3.72-3.68(m, 1 \mathrm{H}$, $\mathrm{NHCH}), 5.60(\mathrm{~s}, \mathrm{br} ., 1 \mathrm{H}, \mathrm{NH}) .{ }^{13} \mathrm{C} \mathrm{NMR}\left(100 \mathrm{MHz}, \mathrm{CDCl}_{3}\right) \delta 23.32\left(\mathrm{CH}_{3}\right), 26.46\left(\mathrm{CH}_{2}\right), 28.09\left(\mathrm{CH}_{2}\right)$, $35.54\left(\mathrm{CH}_{2}\right), 35.68(\mathrm{CH}), 40.40\left(\mathrm{CH}_{2}\right), 42.31(\mathrm{CH}), 52.92(\mathrm{CH}), 169.27(\mathrm{C})$. HRMS (ESI) calculated for $\mathrm{C}_{9} \mathrm{H}_{15} \mathrm{NONa}\left([\mathrm{M}+\mathrm{Na}]^{+}\right):$176.1046. Found 176.1048.

$N$-Bicyclo[2.2.1]hept-2-yl-benzamide (10e): Prepared according to the general procedure (4) using reagent $8 \mathrm{e}(0.10 \mathrm{~g}, 0.35 \mathrm{mmol})$, norbornene $(82 \mathrm{mg}, 0.87 \mathrm{mmol})$, DTBP $(25 \mathrm{mg}, 0.17 \mathrm{mmol})$ and thiophenol $(6.0 \mathrm{mg}, 0.05 \mathrm{mmol})$ in dry benzene $(0.9 \mathrm{ml})$. Purification by FC (pentane / tertbutylmethylether 10:1) provided 10e as a white solid ${ }^{\mathrm{d}}$ (10 mg, $13 \%$ ). IR (neat) 3296br.m, 2958m, 2946s, $2873 w, 1628 s, 1580 m, 1529 s, 1490 m, 1451 m, 1365 w, 1313 s, 1294 m, 1223 w, 1181 w, 1097 m, 1028 m$, $891 w, 836 m, 803 m, 767 w \mathrm{~cm}^{-1} .{ }^{1} \mathrm{H}$ NMR $\left(300 \mathrm{MHz}, \mathrm{CDCl}_{3}\right) \delta 1.58-1.09\left(m, 7 \mathrm{H}, \mathrm{CH}_{2}, \mathrm{CHH}\right), 1.89-1.81$ $(m, 1 \mathrm{H}, \mathrm{CH} H), 2.30-2.27(\mathrm{~m}, 2 \mathrm{H}, \mathrm{CH}), 3.91-3.85(\mathrm{~m}, 1 \mathrm{H}, \mathrm{NHCH}), 6.17$ ( $s$, br., 1H, NH), 7.47-7.34 ( $m$, $3 \mathrm{H}, \mathrm{CH}$-aromatic), 7.75-7.71 ( $\mathrm{m}, 2 \mathrm{H}, \mathrm{CH}$-aromatic). ${ }^{13} \mathrm{C} \mathrm{NMR}\left(75 \mathrm{MHz}, \mathrm{CDCl}_{3}\right) \delta 26.43\left(\mathrm{CH}_{2}\right), 28.07$ $\left(\mathrm{CH}_{2}\right), 35.58\left(\mathrm{CH}_{2}\right), 35.67(\mathrm{CH}), 40.30\left(\mathrm{CH}_{2}\right), 42.33(\mathrm{CH}), 53.28(\mathrm{CH}), 126.78(\mathrm{CH}), 128.35(\mathrm{CH})$, $131.13(\mathrm{CH}), 134.78(\mathrm{C}), 166.83(\mathrm{C})$. HRMS (ESI) calculated for $\mathrm{C}_{14} \mathrm{H}_{17} \mathrm{NONa}\left([\mathrm{M}+\mathrm{Na}]^{+}\right): 238.1202$. Found: 238.1201.

$\mathrm{N}$-Bicyclo[2.2.1]hept-2-yl-2,6-difluorobenzamide (10f): Prepared according to the general procedure (4) using reagent $8 f(0.1 \mathrm{~g}, 0.3 \mathrm{mmol})$, norbornene $(73 \mathrm{mg}, 0.80 \mathrm{mmol})$, DTBP $(23 \mathrm{mg}, 0.15 \mathrm{mmol})$ and thiophenol $(5.0 \mathrm{mg}, 0.05 \mathrm{mmol})$ in dry benzene $(0.8 \mathrm{ml})$. Purification by FC (pentane / tertbutylmethylether 6:1) provided 10f as a white solid (12 mg, $15 \%$ ). IR (neat) 3285br.m, 2976w, 2956m, $2873 w, 1640 s, 1626 s, 1593 m, 1546 s, 1465 s, 1313 s, 1292 m, 1234 m, 1091 w, 1002 s, 933 w, 791 s, 763 m$ $\mathrm{cm}^{-1} .{ }^{1} \mathrm{H}$ NMR (300 MHz, $\left.\mathrm{CDCl}_{3}\right), \delta 1.36-1.09\left(m, 5 \mathrm{H}, \mathrm{CH}_{2}, \mathrm{CHH}\right), 1.59-1.40\left(\mathrm{~m}, 2 \mathrm{H}, \mathrm{CH}_{2}\right), 1.89-1.81$ $\left(m, 1 \mathrm{H}, \mathrm{CH}_{2}, \mathrm{CH} H\right), 2.32-2.28(\mathrm{~m}, 2 \mathrm{H}, \mathrm{CH}), 3.92-3.86(m, 1 \mathrm{H}, \mathrm{NHCH}), 5.98(s, \mathrm{br} ., 1 \mathrm{H}, \mathrm{NH})$ 6.92-6.84 ( $m, 2 \mathrm{H}, \mathrm{CH}$-aromatic), 7.35-7.25 ( $m, 1 \mathrm{H}, \mathrm{CH}$-aromatic). ${ }^{13} \mathrm{C} \mathrm{NMR}\left(75 \mathrm{MHz}, \mathrm{CDCl}_{3}\right) \delta 26.37\left(\mathrm{CH}_{2}\right)$, $28.07\left(\mathrm{CH}_{2}\right), 35.55\left(\mathrm{CH}_{2}\right), 35.68(\mathrm{CH}), 40.19\left(\mathrm{CH}_{2}\right), 42.26(\mathrm{CH}), 53.38(\mathrm{CH}), 111.79(d, J=25.2 \mathrm{~Hz}$, $\mathrm{CH}), 114.58(t, J=20.0 \mathrm{~Hz}, \mathrm{C}), 131.28(t, J=10.2 \mathrm{~Hz}, \mathrm{CH}), 159.42(\mathrm{C}), 159.84\left(d d, J_{l}=7.1 \mathrm{~Hz}, J_{2}=\right.$ $251.9 \mathrm{~Hz}, \mathrm{C})$. HRMS (ESI) calculated for $\mathrm{C}_{14} \mathrm{H}_{15} \mathrm{~F}_{2} \mathrm{NONa}\left([\mathrm{M}+\mathrm{Na}]^{+}\right)$: 274.1014. Found: 274.1004.

$N$-Bicyclo[2.2.1]hept-2-yl-4-methoxybenzamide (10g): Prepared according to the general procedure (4) using reagent $8 \mathrm{~g}(0.1 \mathrm{~g}, 0.3 \mathrm{mmol})$, norbornene $(75 \mathrm{mg}, 0.80 \mathrm{mmol})$, DTBP $(23 \mathrm{mg}, 0.15 \mathrm{mmol})$ and thiophenol $(5.0 \mathrm{mg}, 0.05 \mathrm{mmol})$ in dry benzene $(0.8 \mathrm{ml})$. Purification by FC (pentane / tertbutylmethylether 10:1) provided 10g as a white solid (7 mg, $9 \%$ ). IR (neat) 3296br.m, 2955s, 2870m, $1622 s, 1606 s, 1546 m, 1502 s, 1364 w, 1311 m, 1249 s, 1174 s, 1097 w, 1031 \mathrm{~s}, 938 \mathrm{w}, 842 s, 769 s \mathrm{~cm}^{-1} .{ }^{1} \mathrm{H}$ NMR $\left(300 \mathrm{MHz}, \mathrm{CDCl}_{3}\right) \delta 1.57-1.09\left(m, 7 \mathrm{H}, \mathrm{CH}_{2}, \mathrm{CHH}\right), 1.88-1.80(m, 1 \mathrm{H}, \mathrm{CH} H), 2.29-2.28(m, 2 \mathrm{H}$, $\mathrm{CH}), 3.80\left(s, 3 \mathrm{H}, \mathrm{OCH}_{3}\right), 3.90-3.85(m, 1 \mathrm{H}, \mathrm{NHCH}), 6.07(s$, br., $1 \mathrm{H}, \mathrm{NH}), 6.86(d, 2 \mathrm{H}, J=9.0, \mathrm{CH}-$ aromatic), $7.69\left(d, 2 \mathrm{H}, J=9.0, \mathrm{CH}\right.$-aromatic). ${ }^{13} \mathrm{C} \mathrm{NMR}\left(75 \mathrm{MHz}, \mathrm{CDCl}_{3}\right) \delta 26.45\left(\mathrm{CH}_{2}\right), 28.09\left(\mathrm{CH}_{2}\right)$, $35.59\left(\mathrm{CH}_{2}\right), 35.67(\mathrm{CH}), 40.38\left(\mathrm{CH}_{2}\right), 42.36(\mathrm{CH}), 53.23(\mathrm{CH}), 55.29\left(\mathrm{CH}_{3}\right), 113.52(\mathrm{CH}), 127.05(\mathrm{C})$, 
$128.56(\mathrm{CH}), 131.98(\mathrm{C}), 161.87(\mathrm{C})$. HRMS (ESI) calculated for $\mathrm{C}_{15} \mathrm{H}_{19} \mathrm{NO}_{2} \mathrm{Na}\left([\mathrm{M}+\mathrm{Na}]^{+}\right): 268.1308$. Found: 268.1298.

1-Bicyclo[2.2.1]hept-2-yl-pyrrolidin-2-one (10h): Prepared according to the general procedure (4) using reagent $8 \mathrm{~h}(0.1 \mathrm{~g}, 0.4 \mathrm{mmol})$, norbornene $(94 \mathrm{mg}, 1.0 \mathrm{mmol})$, DTBP $(29 \mathrm{mg}, 0.20 \mathrm{mmol})$ and thiophenol $(6.0 \mathrm{mg}, 0.05 \mathrm{mmol})$ in dry benzene $(1 \mathrm{ml})$. Purification by FC (pentane / tertbutylmethylether 1:2) provided 10h as a colourless oil (16 mg, $22 \%)$. IR (neat) 3453br.w, 2954s, $2871 m, 1976 s, 1492 w, 1495 w, 1423 m, 1287 s, 1228 s, 1048 w, 877 w, 734 w \mathrm{~cm}^{-1} .{ }^{1} \mathrm{H}$ NMR (400 MHz, $\left.\mathrm{CDCl}_{3}\right) \delta 1.21-1.12\left(m, 2 \mathrm{H}, \mathrm{CH}_{2}\right), 1.50-1.29\left(m, 5 \mathrm{H}, \mathrm{CH}_{2}, \mathrm{CHH}\right), 1.73-1.67(m, 1 \mathrm{H}, \mathrm{CH} H), 2.03-1.87$ $\left(m, 2 \mathrm{H}, \mathrm{CH}_{2}\right), 2.16(s$, br., $1 \mathrm{H}, \mathrm{CH}), 2.29(s$, br., $1 \mathrm{H}, \mathrm{CH}), 2.35\left(t, 2 \mathrm{H}, J=8.5 \mathrm{~Hz}, \mathrm{COCH}_{2}\right), 3.43-3.31$ $\left(m, 2 \mathrm{H}, \mathrm{NCH}_{2}\right), 4.05-4.01(\mathrm{~m}, 1 \mathrm{H}, \mathrm{NHCH}) .{ }^{13} \mathrm{C} \mathrm{NMR}\left(100 \mathrm{MHz}, \mathrm{CDCl}_{3}\right) \delta 18.06\left(\mathrm{CH}_{2}\right), 27.66\left(\mathrm{CH}_{2}\right)$, $28.45\left(\mathrm{CH}_{2}\right), 31.38\left(\mathrm{CH}_{2}\right), 36.05(\mathrm{CH}), 36.78\left(\mathrm{CH}_{2}\right), 36.95\left(\mathrm{CH}_{2}\right), 40.36(\mathrm{CH}), 44.17\left(\mathrm{CH}_{2}\right), 54.30(\mathrm{CH})$, 174.65 (C). HRMS (ESI) calculated for $\mathrm{C}_{11} \mathrm{H}_{17} \mathrm{NONa}\left([\mathrm{M}+\mathrm{Na}]^{+}\right)$: 202.1202. Found: 202.1189 .

Cyclopentylcarbamic acid methyl ester (11): Prepared according to the general procedure (4) using reagent $8 \mathbf{a}(0.15 \mathrm{~g}, 0.62 \mathrm{mmol})$, cyclopentene $(0.11 \mathrm{~g}, 1.6 \mathrm{mmol})$, DTBP $(46 \mathrm{mg}, 0.31 \mathrm{mmol})$ and thiophenol $(10 \mathrm{mg}, 0.09 \mathrm{mmol})$ in dry benzene $(1.5 \mathrm{ml})$. Purification by FC (pentane / tertbutylmethylether 8:1) provided 11 as a white solid (43 mg, $48 \%$ ). IR (neat) 3326br.s, 2958s, 2872m, $1722 s, 1538 s, 1454 m, 1360 m, 1252 m, 1106 m, 1050 m, 780 \mathrm{~s} \mathrm{~cm}^{-1} .{ }^{1} \mathrm{H}$ NMR $\left(400 \mathrm{MHz}, \mathrm{CDCl}_{3}\right) \delta 1.39-$ $1.31\left(m, 2 \mathrm{H}, \mathrm{CH}_{2}\right), 1.67-1.50\left(m, 4 \mathrm{H}, \mathrm{CH}_{2}\right), 1.95-1.87\left(m, 2 \mathrm{H}, \mathrm{CH}_{2}\right), 3.62\left(\mathrm{~s}, 3 \mathrm{H}, \mathrm{CO}_{2} \mathrm{CH}_{3}\right), 3.94-3.89$ $(m, 1 \mathrm{H}, \mathrm{NHCH}), 4.68(s$, br., $1 \mathrm{H}, \mathrm{NH}) .{ }^{13} \mathrm{C} \mathrm{NMR}\left(100 \mathrm{MHz}, \mathrm{CDCl}_{3}\right) \delta 23.43\left(\mathrm{CH}_{2}\right), 33.20\left(\mathrm{CH}_{2}\right), 51.79$ $(\mathrm{CH}), 52.79\left(\mathrm{CH}_{3}\right), 156.57(\mathrm{C})$. HRMS (ESI) calculated for $\mathrm{C}_{7} \mathrm{H}_{13} \mathrm{NO}_{2} \mathrm{Na}\left([\mathrm{M}+\mathrm{Na}]^{+}\right)$: 166.0838 . Found: 166.0837 .

Cyclohexylcarbamic acid methyl ester (12): Prepared according to the general procedure (4) using reagent $8 \mathbf{a}(0.15 \mathrm{~g}, 0.62 \mathrm{mmol})$, cyclohexene $(0.13 \mathrm{~g}, 1.6 \mathrm{mmol})$, DTBP $(46 \mathrm{mg}, 0.31 \mathrm{mmol})$ and thiophenol $(10 \mathrm{mg}, 0.09 \mathrm{mmol})$ in dry benzene $(1.5 \mathrm{ml})$. Purification by FC (pentane / tertbutylmethylether 8:1) provided $\mathbf{1 2}$ as a white solid (50 mg, $51 \%$ ). IR (neat) $3345 \mathrm{~s}, 2942 \mathrm{~s}, 2851 \mathrm{~m}$, $1639 s, 1530 s, 1451 w, 1317 m, 1277 m, 1250 m, 1229 s, 1054 s \mathrm{~cm}^{-1} .{ }^{1} \mathrm{H}$ NMR $\left(400 \mathrm{MHz}, \mathrm{CDCl}_{3}\right) \delta 1.17-$ $1.04\left(m, 3 \mathrm{H}, \mathrm{CH}_{2}, \mathrm{CHH}\right), 1.35-1.24\left(m, 2 \mathrm{H}, \mathrm{CH}_{2}\right), 1.68-1.52\left(m, 3 \mathrm{H}, \mathrm{CH}_{2}, \mathrm{CH} H\right), 1.90-1.86(m, 2 \mathrm{H}$, $\left.\mathrm{CH}_{2}\right), 3.44-3.43(\mathrm{~m}, 1 \mathrm{H}, \mathrm{NHCH}), 3.61\left(\mathrm{~s}, 3 \mathrm{H}, \mathrm{CO}_{2} \mathrm{CH}_{3}\right), 6.64(\mathrm{~s}$, br., $1 \mathrm{H}, \mathrm{NH}) .{ }^{13} \mathrm{C} \mathrm{NMR}(100 \mathrm{MHz}$, $\left.\mathrm{CDCl}_{3}\right) \delta 24.72\left(\mathrm{CH}_{2}\right), 25.43\left(\mathrm{CH}_{2}\right), 33.37\left(\mathrm{CH}_{2}\right), 49.82(\mathrm{CH}), 51.71\left(\mathrm{CH}_{3}\right), 156.19(\mathrm{C})$. HRMS (ESI) calculated for $\mathrm{C}_{8} \mathrm{H}_{15} \mathrm{NO}_{2} \mathrm{Na}\left([\mathrm{M}+\mathrm{Na}]^{+}\right): 180.1000$. Found: 180.1017 .

Cyclododecylcarbamic acid methyl ester (13): Prepared according to the general procedure (4) using reagent 8a $(0.1 \mathrm{~g}, 0.4 \mathrm{mmol})$, cyclododecene $(0.42 \mathrm{~g}, 1.0 \mathrm{mmol})$, DTBP $(30 \mathrm{mg}, 0.20 \mathrm{mmol})$ and thiophenol $(7.0 \mathrm{mg}, 0.06 \mathrm{mmol})$ in dry benzene $(1 \mathrm{ml})$. Purification by FC (pentane / tertbutylmethylether 10:1) provided 13 as a white solid (42 mg, $42 \%$ ). IR (neat) 3349br. s, 2932s, 2856m, $1694 s, 1530 s, 1459 m, 1233 w, 1123 w, 1071 w \mathrm{~cm}^{-1} .{ }^{1} \mathrm{H}$ NMR $\left(400 \mathrm{MHz}, \mathrm{CDCl}_{3}\right) \delta 1.67-1.18(m, 22 \mathrm{H}$, $\left.\mathrm{CH}_{2}\right), 3.64\left(s, 3 \mathrm{H}, \mathrm{CO}_{2} \mathrm{CH}_{3}\right), 3.74(s$, br., $1 \mathrm{H} \mathrm{NHCH}), 4.48(s$, br., $1 \mathrm{H}, \mathrm{NH}) .{ }^{13} \mathrm{C} \mathrm{NMR}(100 \mathrm{MHz}$, $\left.\mathrm{CDCl}_{3}\right) \delta 21.39\left(\mathrm{CH}_{2}\right), 23.40\left(\mathrm{CH}_{2}\right), 23.54\left(\mathrm{CH}_{2}\right), 23.66\left(\mathrm{CH}_{2}\right), 23.90\left(\mathrm{CH}_{2}\right), 30.63\left(\mathrm{CH}_{2}\right), 47.86(\mathrm{CH})$, $51.78\left(\mathrm{CH}_{3}\right), 156.53(\mathrm{C})$. HRMS (ESI) calculated for $\mathrm{C}_{14} \mathrm{H}_{27} \mathrm{NO}_{2} \mathrm{Na}\left([\mathrm{M}+\mathrm{Na}]^{+}\right)$: 264.1934. Found: 264.1942.

(2-Methylnonyl)carbamic acid methyl ester (14): Prepared according to the general procedure (4) using reagent 8a $(0.15 \mathrm{~g}, 0.62 \mathrm{mmol})$, 2-methyl-1-nonene $(0.26 \mathrm{~g}, 1.9 \mathrm{mmol})$, DTBP (46 mg, 0.31 $\mathrm{mmol})$ and thiophenol $(14 \mathrm{mg}, 0.12 \mathrm{mmol})$ in dry benzene $(1.5 \mathrm{ml})$. Purification by FC (pentane / tertbutylmethylether 8:1) provided 14 as a colourless oil (62 mg, $46 \%$ ). IR (neat) 3339br.m, 2925s, 2859w, $1708 s, 1537 s, 1460 m, 1257 \mathrm{sm}^{-1} .{ }^{1} \mathrm{H}$ NMR $\left(300 \mathrm{MHz}, \mathrm{CDCl}_{3}\right) \delta 0.91-0.80\left(m, 6 \mathrm{H}, \mathrm{CH}_{3}\right), 1.37-0.98(m$, $\left.12 \mathrm{H}, \mathrm{CH}_{2}\right), 1.63-1.49(m, 1 \mathrm{H}, \mathrm{CH}), 2.98-2.89(m, 1 \mathrm{H}, \mathrm{NHCHH}), 3.13-3.05(m, 1 \mathrm{H}, \mathrm{NHCH}), 3.64(s$, $\left.3 \mathrm{H}, \mathrm{CO}_{2} \mathrm{CH}_{3}\right), 4.71(s$, br., $1 \mathrm{H}, \mathrm{NH}) .{ }^{13} \mathrm{C} \mathrm{NMR}\left(75 \mathrm{MHz}, \mathrm{CDCl}_{3}\right) \delta 14.07\left(\mathrm{CH}_{3}\right), 17.43\left(\mathrm{CH}_{3}\right), 22.63$ $\left(\mathrm{CH}_{2}\right), 26.85\left(\mathrm{CH}_{2}\right), 29.25\left(\mathrm{CH}_{2}\right), 29.79\left(\mathrm{CH}_{2}\right), 31.82\left(\mathrm{CH}_{2}\right), 33.55\left(\mathrm{CH}_{2}\right), 34.15(\mathrm{CH}), 47.05\left(\mathrm{CH}_{2}\right)$, 
$51.96\left(\mathrm{CH}_{3}\right), 157.16(\mathrm{C})$. HRMS (ESI) calculated for $\mathrm{C}_{12} \mathrm{H}_{25} \mathrm{NO}_{2} \mathrm{Na}\left([\mathrm{M}+\mathrm{Na}]^{+}\right)$: 238.1778. Found: 238.1762 .

Cyclohexylmethylcarbamic acid methyl ester (15): Prepared according to the general procedure (4) using reagent $8 \mathbf{a}(0.15 \mathrm{~g}, 0.62 \mathrm{mmol})$, methylene-cyclohexane $(0.38 \mathrm{~g}, 1.9 \mathrm{mmol})$, DTBP (46 mg, 0.31 $\mathrm{mmol})$ and thiophenol $(10 \mathrm{mg}, 0.09 \mathrm{mmol})$ in dry benzene $(1.5 \mathrm{ml})$. Purification by FC (pentane / tertbutylmethylether 6:1) provided $\mathbf{1 5}$ as colourless oil which was solidified upon stand at low temperature (88 mg, $51 \%$ ). IR (neat) 3336br.m, 2924s, 2825m, 1705s, 1538s, 1449m, 1254s $\mathrm{cm}^{-1}$. ${ }^{1} \mathrm{H}$ NMR (400 $\mathrm{MHz}_{\mathrm{CDCl}} \mathrm{CD}_{3} \delta$ 0.93-0.84 ( $\left.m, 2 \mathrm{H}, \mathrm{CH}_{2}\right), 1.26-1.07\left(\mathrm{~m}, 3 \mathrm{H}, \mathrm{CH}_{2}\right), 1.41(\mathrm{~s}$, br., $1 \mathrm{H}, \mathrm{CH}), 1.82-1.62(\mathrm{~m}$, $\left.5 \mathrm{H}, \mathrm{CH}_{2}\right), 2.99\left(s\right.$, br., $\left.2 \mathrm{H}, \mathrm{NHCH}_{2}\right), 3.64\left(s, 3 \mathrm{H}, \mathrm{CO}_{2} \mathrm{CH}_{3}\right), 4.74(s$, br., $1 \mathrm{H}, \mathrm{NH}) .{ }^{13} \mathrm{C} \mathrm{NMR}(100 \mathrm{MHz}$, $\left.\mathrm{CDCl}_{3}\right) \delta 25.77\left(\mathrm{CH}_{2}\right), 26.38\left(\mathrm{CH}_{2}\right), 30.62\left(\mathrm{CH}_{2}\right), 38.24(\mathrm{CH}), 47.32\left(\mathrm{CH}_{2}\right), 51.92\left(\mathrm{CH}_{3}\right), 157.18(\mathrm{C})$. HRMS (ESI) calculated for $\mathrm{C}_{9} \mathrm{H}_{17} \mathrm{NO}_{2} \mathrm{Na}\left([\mathrm{M}+\mathrm{Na}]^{+}\right)$: 194.1151 . Found: 194.1145.

[5-(tert-Butyldimethylsilanyloxy)pentyl]carbamic acid methyl ester (16a): Prepared according to the general procedure $(\mathbf{4})$ using reagent $8 \mathbf{a}(0.15 \mathrm{~g}, 0.62 \mathrm{mmol})$, tert-butyl-dimethyl-pent-4-enyloxy-silane $(0.38 \mathrm{~g}, 1.9 \mathrm{mmol})$, DTBP $(46 \mathrm{mg}, 0.31 \mathrm{mmol})$ and thiophenol $(10 \mathrm{mg}, 0.09 \mathrm{mmol})$ in dry benzene $(1.5$ ml). Purification by FC (pentane / tert-butylmethylether 6:1) provided 16a as a colourless oil with another regioisomer in 1:10 ratio (ratio was determined by ${ }^{1} \mathrm{H}$ NMR spectroscopy) (88 $\mathrm{mg}, 51 \%$ (combined yield of both isomers)). Physical data of the major isomer is given. IR (neat) $3342 \mathrm{br} . \mathrm{m}$, $2932 m, 2858 w, 1706 s, 1535 m, 1463 w, 1253 s, 1099 s, 835 s .776 s \mathrm{~cm}^{-1} .{ }^{1} \mathrm{H}$ NMR $\left(300 \mathrm{MHz}, \mathrm{CDCl}_{3}\right) \delta$ $0.02\left(s, 6 \mathrm{H}, \mathrm{SiCH}_{3}\right), 0.95\left(s, 9 \mathrm{H}, \mathrm{C}\left(\mathrm{CH}_{3}\right)_{3}\right), 1.18-1.06\left(m, 4 \mathrm{H}, \mathrm{CH}_{2}\right), 1.38-1.28\left(m, 2 \mathrm{H}, \mathrm{CH}_{2}\right), 2.94-2.86$ $\left(m, 2 \mathrm{H}, \mathrm{NHCH}_{2}\right), 3.42-3.37\left(m, 2 \mathrm{H}, \mathrm{OCH}_{2}\right), 3.44\left(s, 3 \mathrm{H}, \mathrm{CO}_{2} \mathrm{CH}_{3}\right), 4.12(s$, br., $1 \mathrm{H}, \mathrm{NH}) .{ }^{13} \mathrm{C}(75 \mathrm{MHz}$, $\left.\mathrm{CDCl}_{3}\right) \delta-5.17\left(\mathrm{CH}_{3}\right), 18.49(\mathrm{C}), 23.16\left(\mathrm{CH}_{2}\right), 26.14\left(\mathrm{CH}_{3}\right), 30.02\left(\mathrm{CH}_{2}\right), 32.70\left(\mathrm{CH}_{2}\right), 41.12\left(\mathrm{CH}_{2}\right)$, $51.59\left(\mathrm{CH}_{3}\right), 63.02\left(\mathrm{CH}_{2}\right), 156.84(\mathrm{C})$. HRMS (ESI) calculated for $\mathrm{C}_{13} \mathrm{H}_{29} \mathrm{NO}_{3} \mathrm{SiNa}\left([\mathrm{M}+\mathrm{Na}]^{+}\right)$: 298.1809. Found: 298.1804.

(10-Hydroxydecyl)carbamic acid methyl ester (17a): Prepared according to the general procedure (4) using reagent 8a $(0.1 \mathrm{~g}, 0.4 \mathrm{mmol})$, dec-9-en-1-ol $(0.16 \mathrm{~g}, 1.0 \mathrm{mmol})$, DTBP (30 $\mathrm{mg}, 0.20 \mathrm{mmol})$ and thiophenol $(7.0 \mathrm{mg}, 0.08 \mathrm{mmol})$ in dry benzene $(1 \mathrm{ml})$ Purification by FC (pentane / tertbutylmethylether 10:1) provided 17a a as white solid with another regioisomer in 1:10 ratio (ratio was determined by ${ }^{1} \mathrm{H}$ NMR spectroscopy) (44 mg, $46 \%$ (combined yield of both isomers)). Physical data of the major isomer is given. IR (neat) $3349 \mathrm{br} . s, 2923 \mathrm{~s}, 2852 \mathrm{~s}, 1689 \mathrm{~s}, 1531 \mathrm{~s}, 1462 \mathrm{~m}, 1354 \mathrm{w}, 1278 \mathrm{~m}$, $1254 \mathrm{~s}, 1055 \mathrm{~s}, 1012 \mathrm{~m} \mathrm{~cm}^{-1} .{ }^{1} \mathrm{H}$ NMR $\left(400 \mathrm{MHz} \mathrm{CDCl}_{3}\right) \delta 1.48-1.12\left(\mathrm{~m}, 16 \mathrm{H}, \mathrm{CH}_{2}\right), 3.05-3.02(\mathrm{~m}, 2 \mathrm{H}$, $\left.\mathrm{NHCH}_{2}\right), 3.51\left(t, 2 \mathrm{H}, J=6.6 \mathrm{~Hz}, \mathrm{OCH}_{2}\right), 3.54\left(s, 3 \mathrm{H}, \mathrm{CO}_{2} \mathrm{CH}_{3}\right), 4.53(s$, br., $1 \mathrm{H}, \mathrm{NH}) .{ }^{13} \mathrm{C} \mathrm{NMR}(100$ $\left.\mathrm{MHz}, \mathrm{CDCl}_{3}\right) \delta 25.69\left(\mathrm{CH}_{2}\right), 26.66\left(\mathrm{CH}_{2}\right), 29.18\left(\mathrm{CH}_{2}\right), 29.33\left(\mathrm{CH}_{2}\right), 29.38\left(\mathrm{CH}_{2}\right), 29.44\left(\mathrm{CH}_{2}\right), 29.99$ $\left(\mathrm{CH}_{2}\right), 32.77\left(\mathrm{CH}_{2}\right), 41.11\left(\mathrm{CH}_{2}\right), 51.98\left(\mathrm{CH}_{3}\right), 63.02\left(\mathrm{CH}_{2}\right), 157.07(\mathrm{C})$. HRMS (ESI) calculated for $\mathrm{C}_{12} \mathrm{H}_{25} \mathrm{NO}_{3} \mathrm{Na}\left([\mathrm{M}+\mathrm{Na}]^{+}\right): 254.1727$. Found: 254.1724.

[1-(tert-Butyldimethylsilanyloxymethyl)2-methyl-propyl]carbamic acid methyl ester (18): Prepared according to the general procedure (4) using reagent $8 \mathbf{a}(0.1 \mathrm{~g}, 0.4 \mathrm{mmol})$, tert-butyl-dimethyl-(3methyl-but-2-enyloxy)-silane $(0.21 \mathrm{~g}, 1.0 \mathrm{mmol})$, DTBP $(30 \mathrm{mg}, 0.20 \mathrm{mmol})$ and thiophenol $(9.0 \mathrm{mg}$, $0.08 \mathrm{mmol}$ ) in dry benzene $(1 \mathrm{ml})$. Purification by FC (pentane / tert-butylmethylether 10:1) provided 18 as colourless oil (47 mg, $41 \%$ ). IR (neat) 3333br.w, 2958s, 1705s, 1536s, 1465m, 1255s, 1102m, 837s, $777 s \mathrm{~cm}^{-1} .{ }^{1} \mathrm{H}$ NMR $\left(400 \mathrm{MHz}, \mathrm{CDCl}_{3}\right) \delta 0.04(s, 6 \mathrm{H}, \mathrm{SiCH}), 0.88\left(s, 9 \mathrm{H}, \mathrm{SiC}\left(\mathrm{CH}_{3}\right)_{3}\right), 0.94-0.90(m$, $\left.6 \mathrm{H}, \mathrm{CH}\left(\mathrm{CH}_{3}\right)_{2}\right), 1.92-1.82\left(m, 1 \mathrm{H}, \mathrm{CH}\left(\mathrm{CH}_{3}\right)_{2}\right), 3.41(s$, br., $1 \mathrm{H}, \mathrm{CHH}), 3.59-3.55\left(d d, 1 \mathrm{H}, J_{1}=4.2 \mathrm{~Hz}, J_{2}\right.$ $=10.1 \mathrm{~Hz}, \mathrm{CH} H), 3.71-3.63\left(\mathrm{~m}, 4 \mathrm{H}, \mathrm{CO}_{2} \mathrm{CH}_{3}, \mathrm{CHOTBS}\right), 4.82(s$, br., $1 \mathrm{H}, \mathrm{NH}) .{ }^{13} \mathrm{C}$ NMR $(100 \mathrm{MHz}$, $\left.\mathrm{CDCl}_{3}\right) \delta-5.54\left(\mathrm{CH}_{3}\right), 18.20(\mathrm{C}), 18.63(\mathrm{CH}), 25.82\left(\mathrm{CH}_{3}\right), 29.05\left(\mathrm{CH}_{3}\right), 51.96\left(\mathrm{CH}_{3}\right), 57.61(\mathrm{CH})$, $63.04\left(\mathrm{CH}_{2}\right), 157.00(\mathrm{C})$. HRMS (ESI) calculated for $\mathrm{C}_{13} \mathrm{H}_{29} \mathrm{NO}_{3} \mathrm{SiNa}\left([\mathrm{M}+\mathrm{Na}]^{+}\right)$: 298.1809 . Found: 298.1798.

Acetic acid 3-methoxycarbonylaminopropyl ester (19): Prepared according to the general procedure (4) using reagent 8a $(0.15 \mathrm{~g}, 0.62 \mathrm{mmol})$, acetic acid allyl ester $(0.19 \mathrm{~g}, 1.9 \mathrm{mmol})$, DTBP (46 mg, 0.31 
mmol) and thiophenol $(7.0 \mathrm{mg}, 0.06 \mathrm{mmol})$ in dry benzene $(1.5 \mathrm{ml})$. Purification by FC (pentane / tertbutylmethylether 1:1) provided 19 as a colourless oil (57 mg, $52 \%$ ). IR (neat) 3355br.m, 2956m, 1733s, $1534 s, 1451 w, 1368 m, 1243 s, 1043 m, 779 w \mathrm{~cm}^{-1} .{ }^{1} \mathrm{H}$ NMR $\left(300 \mathrm{MHz}, \mathrm{CDCl}_{3}\right) \delta 1.85-1.77(m, 2 \mathrm{H}$, $\left.\mathrm{CH}_{2}\right), 2.04\left(s, 3 \mathrm{H}, \mathrm{COCH}_{3}\right), 3.26-3.20\left(m, 2 \mathrm{H}, \mathrm{NHCH}_{2}\right), 3.64\left(s, 3 \mathrm{H}, \mathrm{CO}_{2} \mathrm{CH}_{3}\right), 4.11(t, 2 \mathrm{H}, J=6.1 \mathrm{~Hz}$, $\left.\mathrm{CH}_{2} \mathrm{OAc}\right), 4.94(s$, br., $1 \mathrm{H}, \mathrm{NH}) .{ }^{13} \mathrm{C}$ NMR $\left(75 \mathrm{MHz}, \mathrm{CDCl}_{3}\right) \delta 20.88\left(\mathrm{CH}_{3}\right), 28.97\left(\mathrm{CH}_{2}\right), 37.80\left(\mathrm{CH}_{2}\right)$, $52.02\left(\mathrm{CH}_{3}\right), 61.76\left(\mathrm{CH}_{2}\right), 157.00(\mathrm{C}), 171.15(\mathrm{C})$. HRMS (ESI) calculated for $\mathrm{C}_{7} \mathrm{H}_{13} \mathrm{NO}_{4} \mathrm{Na}\left([\mathrm{M}+\mathrm{Na}]^{+}\right)$: 198.0737. Found: 198.0734.

(3-iso-Propyloxylsilanylpropyl)carbamic acid methyl ester (20): Prepared according to the general procedure (4) using reagent $\mathbf{8 a}(0.15 \mathrm{~g}, 0.62 \mathrm{mmol})$, allyltriisopropoxysilane $(0.39 \mathrm{~g}, 1.6 \mathrm{mmol})$, DTBP (46 mg, $0.31 \mathrm{mmol})$ and thiophenol $(10 \mathrm{mg}, 0.09 \mathrm{mmol})$ in dry benzene $(1.5 \mathrm{ml})$. Purification by FC (pentane / tert-butylmethylether 10:1) provided 20 as a colourless oil (93 mg, 46\%). IR (neat) 3344br.m, $2973 s, 1708 s, 1533 s, 1377 m, 1251 m, 1173 m, 1122 s, 1039 s, 886 m, 776 m \mathrm{~cm}^{-1}$. ${ }^{1} \mathrm{H}$ NMR (300 MHz, $\left.\mathrm{CDCl}_{3}\right)$ 0.65-0.59 (m, 2H, SiCH $\mathrm{S}_{2}, 1.27\left(d, 18 \mathrm{H}, J=6.1 \mathrm{~Hz}, \mathrm{CH}\left(\mathrm{CH}_{3}\right)_{2}\right), 1.76-1.66\left(m, 2 \mathrm{H}, \mathrm{CH}_{2}\right), 3.24-$ $3.17\left(m, 2 \mathrm{H}, \mathrm{NHCH}_{2}\right), 3.56\left(s, 3 \mathrm{H}, \mathrm{CO}_{2} \mathrm{CH}_{3}\right), 4.28$ (sept., $\left.1 \mathrm{H}, J=6.1 \mathrm{~Hz}, \mathrm{CH}\left(\mathrm{CH}_{3}\right)_{2}\right), 4.77(s$, br., $1 \mathrm{H}$, $\mathrm{NH}) .{ }^{13} \mathrm{C}$ NMR $\left(75 \mathrm{MHz}, \mathrm{CDCl}_{3}\right) \delta 9.46\left(\mathrm{CH}_{2}\right), 24.01\left(\mathrm{CH}_{2}\right), 25.75\left(\mathrm{CH}_{3}\right), 43.78\left(\mathrm{CH}_{2}\right), 51.54\left(\mathrm{CH}_{3}\right)$, $65.17(\mathrm{CH}), 156.94(\mathrm{C})$. HRMS (ESI) calculated for $\mathrm{C}_{14} \mathrm{H}_{31} \mathrm{NO}_{5} \mathrm{SiNa}\left([\mathrm{M}+\mathrm{Na}]^{+}\right)$: 344.1864 . Found: 344.1860 .

(3-Methoxycarbonylaminopropyl)phosphonic acid dimethyl ester (21): Prepared according to the general procedure (4) using reagent $8 \mathbf{a}(0.1 \mathrm{~g}, 0.4 \mathrm{mmol})$, allyl-phosphonic acid dimethyl ester $(0.16 \mathrm{~g}$, $1.0 \mathrm{mmol})$, DTBP (30 mg, $0.20 \mathrm{mmol})$ and thiophenol $(7.0 \mathrm{mg}, 0.06 \mathrm{mmol})$ in dry benzene $(1 \mathrm{ml})$. Purification by FC (pentane / tert-butylmethylether 10:1) provided 21 as a colourless oil (30 mg, $32 \%)$. IR (neat) $3417 \mathrm{br} . m, 2956 \mathrm{w}, 1708 \mathrm{~s}, 1541 \mathrm{~m}, 1455 \mathrm{w}, 1255 \mathrm{~s}, 1036 \mathrm{~s}, 823 \mathrm{w} \mathrm{cm}^{-1} .{ }^{1} \mathrm{H}$ NMR (400 MHz, $\left.\mathrm{CDCl}_{3}\right) \delta$ 1.85-1.71 ( $\left.m, 4 \mathrm{H}, \mathrm{CH}_{2}\right), 3.22\left(s\right.$, br., $\left.2 \mathrm{H}, \mathrm{NHCH}_{2}\right), 3.64\left(s, 3 \mathrm{H}, \mathrm{CO}_{2} \mathrm{CH}_{3}\right), 3.70\left(s, 3 \mathrm{H}, \mathrm{OCH}_{3}\right)$, $3.73\left(s, 3 \mathrm{H}, \mathrm{OCH}_{3}\right), 5.11(s$, br., $1 \mathrm{H}, \mathrm{NH}) .{ }^{13} \mathrm{C} \mathrm{NMR}\left(100 \mathrm{MHz}, \mathrm{CDCl}_{3}\right) \delta 21.93\left(d, J=142.5 \mathrm{~Hz}, \mathrm{CH}_{2}\right)$, $22.98\left(d, J=4.9 \mathrm{~Hz}, \mathrm{CH}_{2}\right), 41.16\left(d, J=16.4 \mathrm{~Hz}, \mathrm{CH}_{2}\right), 52.03\left(\mathrm{CH}_{3}\right), 52.35\left(d, J=6.6 \mathrm{~Hz}, \mathrm{CH}_{3}\right), 157.09$ (C). HRMS (ESI) calculated for $\mathrm{C}_{7} \mathrm{H}_{16} \mathrm{NO}_{5} \mathrm{PNa}\left([\mathrm{M}+\mathrm{Na}]^{+}\right)$: 248.0658. Found: 248.0639.

2-(3-Methoxycarbonylaminopropyl)malonic acid diethyl ester (22): Prepared according to the general procedure (4) using reagent $\mathbf{8 a}(0.15 \mathrm{~g}, 0.62 \mathrm{mmol})$, 2-allyl-malonic acid diethyl ester $(0.37 \mathrm{~g}$, $1.9 \mathrm{mmol})$, DTBP (46 mg, $0.31 \mathrm{mmol})$ and thiophenol $(14 \mathrm{mg}, 0.12 \mathrm{mmol})$ in dry benzene $(1.5 \mathrm{ml})$. Purification by FC (pentane / tert-butylmethylether 6:1) provided 22 as a colourless oil (68 mg, $40 \%)$. IR (neat) 3386br.w, 2982m, 1728s, 1532m, 1450w, 1371w, 1254s, 1029m cm ${ }^{-1} .{ }^{1} \mathrm{H}$ NMR (300 MHz, $\left.\mathrm{CDCl}_{3}\right) \delta 1.24\left(t, 3 \mathrm{H}, J=7.1 \mathrm{~Hz}, \mathrm{CO}_{2} \mathrm{CH}_{2} \mathrm{CH}_{3}\right), 1.57-1.45\left(m, 2 \mathrm{H}, \mathrm{CH}_{2}\right), 1.93-1.85\left(m, 2 \mathrm{H}, \mathrm{CH}_{2}\right), 3.20-$ $3.14\left(m, 2 \mathrm{H}, \mathrm{NHCH}_{2}\right), 3.30\left(t, 1 \mathrm{H}, J=7.4 \mathrm{~Hz}, \mathrm{CH}\left(\mathrm{CO}_{2} \mathrm{Et}\right)_{2}\right), 3.63\left(s, 3 \mathrm{H}, \mathrm{CO}_{2} \mathrm{CH}_{3}\right), 4.20-4.13(d q, 4 \mathrm{H}$, $\left.J_{1}=0.9 \mathrm{~Hz}, J_{2}=7.1 \mathrm{~Hz}, \mathrm{CO}_{2} \mathrm{CH}_{2} \mathrm{CH}_{3}\right), 4.82(s$, br., $1 \mathrm{H}, \mathrm{NH}) .{ }^{13} \mathrm{C} \mathrm{NMR}\left(75 \mathrm{MHz}, \mathrm{CDCl}_{3}\right) \delta 14.00$ $\left(\mathrm{CH}_{3}\right), 25.72\left(\mathrm{CH}_{2}\right), 27.60\left(\mathrm{CH}_{2}\right), 40.42\left(\mathrm{CH}_{2}\right), 51.49(\mathrm{CH}), 51.97\left(\mathrm{CH}_{3}\right), 61.39\left(\mathrm{CH}_{2}\right), 156.97(\mathrm{C})$, 169.20 (C). HRMS (ESI) calculated for $\mathrm{C}_{12} \mathrm{H}_{21} \mathrm{NO}_{6} \mathrm{Na}\left([\mathrm{M}+\mathrm{Na}]^{+}\right)$: 298.1261. Found: 298.1260.

(2-Phenyl-propyl)carbamic acid methyl ester (23): Prepared according to the general procedure (4) using reagent $8 \mathbf{a}(0.1 \mathrm{mg}, 0.4 \mathrm{mmol}), \beta$-methylstyrene $(0.12 \mathrm{mg}, 1.0 \mathrm{mmol})$, DTBP $(30 \mathrm{mg}, 0.20 \mathrm{mmol})$ and thiophenol $(7.0 \mathrm{mg}, 0.06 \mathrm{mmol})$ in dry benzene $(1 \mathrm{ml})$. Purification by FC (pentane / tertbutylmethylether 10:1) provided 23 as a colourless oil (39 mg, $48 \%$ ). IR (neat) 3336br.m, 2963m, $1704 s, 1527 s, 1259 s, 1010 m, 762 m, 700 \mathrm{sm}^{-1} .{ }^{1} \mathrm{H}$ NMR $\left(300 \mathrm{MHz}, \mathrm{CDCl}_{3}\right) \delta 1.27(d, 3 \mathrm{H}, J=7.2 \mathrm{~Hz}$, $\left.\mathrm{CH}_{3}\right), 2.98-2.83(m, 1 \mathrm{H}, \mathrm{CH}$-benzylic), 3.27-3.19 $(m, 1 \mathrm{H}, \mathrm{NHCHH}), 3.51-3.38(m, 1 \mathrm{H}, \mathrm{NHCH}), 3.62$ $\left(s, 3 \mathrm{H}, \mathrm{CO}_{2} \mathrm{CH}_{3}\right), 4.59$ (s, br., $\left.1 \mathrm{H}, \mathrm{NH}\right), 7.26-7.18$ (m, 3H, $\mathrm{CH}$-aromatic), 7.35-7.30 (m, 2H, CHaromatic). ${ }^{13} \mathrm{C}$ NMR $\left(75 \mathrm{MHz}, \mathrm{CDCl}_{3}\right) \delta 19.09\left(\mathrm{CH}_{3}\right), 40.04(\mathrm{CH}), 47.69\left(\mathrm{CH}_{2}\right), 52.00\left(\mathrm{CH}_{3}\right), 126.65$ $(\mathrm{CH}), 127.15(\mathrm{CH}), 128.64(\mathrm{CH}), 143.91(\mathrm{C}), 156.95(\mathrm{C})$. HRMS (ESI) calculated for $\mathrm{C}_{11} \mathrm{H}_{15} \mathrm{NO}_{2} \mathrm{Na}$ $\left([\mathrm{M}+\mathrm{Na}]^{+}\right): 216.0995$. Found: 216.0985 . 
[2-(tert-Butyldimethylsilanyloxy)-2-phenylethyl]carbamic acid methyl ester (24): Prepared according to the general procedure (4) using reagent 8a $(0.1 \mathrm{~g}, 0.4 \mathrm{mmol})$, tert-butyl-dimethyl-(1phenyl-vinyloxy)-silane (0.34 g, $0.80 \mathrm{mmol})$, DTBP $(30 \mathrm{mg}, 0.20 \mathrm{mmol})$ and thiophenol $(9.0 \mathrm{mg}, 0.08$ $\mathrm{mmol}$ ) in dry benzene $(1 \mathrm{ml})$. Purification by FC (pentane / tert-butylmethylether 5:1) provided $\mathbf{2 4}$ as a colourless oil (90 mg, $70 \%$ ). IR (neat) 3356br. m, 2954s, 2857m, 1716s, 1518s, 1468m, 1257s, 1098s, $1007 w, 836 s, 778 s, 701 s \mathrm{~cm}^{-1} .{ }^{1} \mathrm{H}$ NMR $\left(300 \mathrm{MHz}, \mathrm{C}_{6} \mathrm{D}_{6}\right) \delta-0.12\left(s, 3 \mathrm{H}, \mathrm{SiCH}_{3}\right), 0.02\left(s, 3 \mathrm{H}, \mathrm{SiCH}_{3}\right)$, $0.91\left(s, 9 \mathrm{H}, \mathrm{SiC}\left(\mathrm{CH}_{3}\right)_{3}\right), 3.13-3.02(m, 1 \mathrm{H}, \mathrm{NHCHH}), 3.39-3.33(m, 1 \mathrm{H}, \mathrm{NHCH}), 3.44(s, 3 \mathrm{H}$, $\mathrm{CO}_{2} \mathrm{CH}_{3}$ ), 4.57 ( $s$, br., $\left.1 \mathrm{H}, \mathrm{NH}\right), 4.79-4.75$ ( $\left.m, 1 \mathrm{H}, \mathrm{CHOTBS}\right), 7.12-7.01$ ( $m, 3 \mathrm{H}, \mathrm{CH}$-aromatic), 7.23$7.18\left(\mathrm{~m}, 2 \mathrm{H}, \mathrm{CH}\right.$-aromatic). ${ }^{13} \mathrm{C} \mathrm{NMR}\left(75 \mathrm{MHz}, \mathrm{CDCl}_{3}\right) \delta-5.13\left(\mathrm{CH}_{3}\right),-4.77\left(\mathrm{CH}_{3}\right), 18.15(\mathrm{C}), 25.76$ $\left(\mathrm{CH}_{2}\right), 49.36\left(\mathrm{CH}_{3}\right), 52.09(\mathrm{CH}), 73.69(\mathrm{CH}), 125.95(\mathrm{CH}), 127.55(\mathrm{CH}), 128.23(\mathrm{CH}), 142.11(\mathrm{C})$, 156.98 (C). HRMS (ESI) calculated for $\mathrm{C}_{16} \mathrm{H}_{27} \mathrm{NO}_{3} \mathrm{SiNa}\left([\mathrm{M}+\mathrm{Na}]^{+}\right)$: 332.1652. Found: 332.1646.

[2-(4-Bromophenyl)-2-(tert-butyldimethylsilanyloxy)ethyl]carbamic acid methyl ester (25): Prepared according to the general procedure (4) using reagent 8a $(0.1 \mathrm{~g}, 0.4 \mathrm{mmol})$, [1-(4-bromophenyl)-vinyloxy]-tert-butyl-dimethyl-silane (0.33 g, $1.0 \mathrm{mmol})$, DTBP (30 $\mathrm{mg}, 0.20 \mathrm{mmol})$ and thiophenol $(9.0 \mathrm{mg}, 0.08 \mathrm{mmol})$ in dry benzene $(1 \mathrm{ml})$. Purification by FC (pentane / tertbutylmethylether 10:1) provided 25 as a colourless oil (100 mg, 62\%). IR (neat) 3357 br.m, 2954m, $2857 w, 1715 s, 1517 m, 1258 s, 1092 s, 1009 m, 836 s, 777 s \mathrm{~cm}^{-1} .{ }^{1} \mathrm{H}$ NMR $\left(400 \mathrm{MHz}, \mathrm{CDCl}_{3}\right) \delta-0.10(s$, $\left.3 \mathrm{H}, \mathrm{SiCH}_{3}\right), 0.03\left(s, 3 \mathrm{H}, \mathrm{SiCH}_{3}\right), 0.07\left(s, 9 \mathrm{H}, \mathrm{SiC}\left(\mathrm{CH}_{3}\right)_{3}\right), 3.17-3.10(m, 1 \mathrm{H}, \mathrm{NHCHH}), 3.42-3.36(m$, $1 \mathrm{H}, \mathrm{NHCH} H), 3.65\left(s, 3 \mathrm{H}, \mathrm{CO}_{2} \mathrm{CH}_{3}\right), 4.75-4.72(m, 1 \mathrm{H}, \mathrm{CHOTBS}), 4.90(s, \mathrm{br}, 1 \mathrm{H}, \mathrm{NH}), 7.20(d, 2 \mathrm{H}, J$ $=8.4 \mathrm{~Hz}, \mathrm{CH}$-aromatic), $7.45\left(d, 2 \mathrm{H}, J=8.4 \mathrm{~Hz}, \mathrm{CH}\right.$-aromatic). ${ }^{13} \mathrm{C} \mathrm{NMR}\left(100 \mathrm{MHz}, \mathrm{CDCl}_{3}\right) \delta-5.11$ $\left(\mathrm{CH}_{3}\right),-4.77\left(\mathrm{CH}_{3}\right), 18.13(\mathrm{C}), 25.74\left(\mathrm{CH}_{3}\right), 49.27\left(\mathrm{CH}_{2}\right), 52.13\left(\mathrm{CH}_{3}\right), 73.16(\mathrm{CH}), 121.42(\mathrm{C}), 127.69$ $(\mathrm{CH}), 131.43(\mathrm{CH}), 141.29(\mathrm{C}), 156.96(\mathrm{C})$. HRMS (ESI) calculated for $\mathrm{C}_{16} \mathrm{H}_{26} \mathrm{BrNO}_{3} \mathrm{SiNa}\left([\mathrm{M}+\mathrm{Na}]^{+}\right)$: 412.0739. Found: 412.0734.

[2-(tert-Butyldimethylsilanyloxy)-2-(3,4-dimethoxyphenyl)ethyl]carbamic acid methyl ester (26): Prepared according to the general procedure (4) using reagent $\mathbf{8 a}(0.1 \mathrm{~g}, 0.4 \mathrm{mmol})$, tert-butyl-[1-(3,4dimethoxy-phenyl)-vinyloxy]-dimethyl-silane $(0.31 \mathrm{mg}, 1.0 \mathrm{mmol})$, DTBP $(30 \mathrm{mg}, 0.20 \mathrm{mmol})$ and thiophenol $(9.0 \mathrm{mg}, 0.08 \mathrm{mmol})$ in dry benzene $(1 \mathrm{ml})$. Purification by FC (pentane / tertbutylmethylether 10:1) provided 26 as a colourless oil (81 mg, $53 \%$ ). IR (neat) 3390br.m, 2943s, 1717s, $1515 s, 1461 m, 1260 s, 1148 m, 1092 s, 1026 m, 834 s, 775 m \mathrm{~cm}^{-1} .{ }^{1} \mathrm{H}$ NMR $\left(400 \mathrm{MHz}, \mathrm{CDCl}_{3}\right) \delta-0.21(s$, $\left.3 \mathrm{H}, \mathrm{SiCH}_{3}\right),-0.07\left(s, 3 \mathrm{H}, \mathrm{SiCH}_{3}\right), 0.77\left(s, 9 \mathrm{H}, \mathrm{SiC}\left(\mathrm{CH}_{3}\right)_{3}\right), 3.07-3.00(m, 1 \mathrm{H}, \mathrm{NHCHH}), 3.35-3.28(m$, $1 \mathrm{H}, \mathrm{NHCH} H), 3.55\left(s, 3 \mathrm{H}, \mathrm{CO}_{2} \mathrm{CH}_{3}\right), 3.75\left(s, 6 \mathrm{H}, \mathrm{OCH}_{3}\right), 4.61-4.58(m, 1 \mathrm{H}, \mathrm{CHOTBS}), 4.79(s$, br., $1 \mathrm{H}$, $\mathrm{NH}), 6.76-6.68\left(\mathrm{~m}, 3 \mathrm{H}, \mathrm{CH}\right.$-aromatic). ${ }^{13} \mathrm{C} \mathrm{NMR}\left(100 \mathrm{MHz}, \mathrm{CDCl}_{3}\right) \delta-5.12\left(\mathrm{CH}_{3}\right),-4.74\left(\mathrm{CH}_{3}\right), 18.15$ (C), $25.75\left(\mathrm{CH}_{3}\right), 49.45\left(\mathrm{CH}_{2}\right), 52.04\left(\mathrm{CH}_{3}\right), 55.80\left(\mathrm{CH}_{3}\right), 55.85\left(\mathrm{CH}_{3}\right), 73.49(\mathrm{CH}), 109.07(\mathrm{CH})$, $110.89(\mathrm{CH}), 118.19(\mathrm{CH}), 134.86(\mathrm{C}), 148.38(\mathrm{C}), 148.89(\mathrm{C}), 156.97(\mathrm{C})$. HRMS (ESI) calculated for $\mathrm{C}_{18} \mathrm{H}_{31} \mathrm{NO}_{5} \mathrm{SiNa}\left([\mathrm{M}+\mathrm{Na}]^{+}\right): 392.1864$. Found: 392.1853.

(2-tert-Butoxyethyl)carbamic acid methyl ester (27): Prepared according to the general procedure (4) using reagent $8 \mathbf{a}(0.15 \mathrm{~g}, 0.62 \mathrm{mmol}), 2$-methyl-2-vinyloxy-propane $(0.16 \mathrm{~g}, 1.6 \mathrm{mmol})$, DTBP (46 mg, $0.31 \mathrm{mmol})$ and thiophenol $(14 \mathrm{mg}, 0.12 \mathrm{mmol})$ in dry benzene $(1.5 \mathrm{ml})$. Purification by FC (pentane / tert-butylmethylether 6:1) provided 27 as a colourless oil (58 mg, $53 \%$ ). IR (neat) 3342br.m, 2975s, $1715 s, 1534 s, 1363 s, 1257 s, 1195 s, 1091 s, 779 m \mathrm{~cm}^{-1} .{ }^{1} \mathrm{H}$ NMR $\left(400 \mathrm{MHz}, \mathrm{CDCl}_{3}\right) \delta 1.16(s, 9 \mathrm{H}$, $\left.\mathrm{C}\left(\mathrm{CH}_{3}\right)_{3}\right), 3.31-3.29\left(m, 2 \mathrm{H}, \mathrm{NHCH}_{2}\right), 3.40\left(t, 2 \mathrm{H}, J=5.1 \mathrm{~Hz}, \mathrm{OCH}_{2}\right), 3.65\left(s, 3 \mathrm{H}, \mathrm{CO}_{2} \mathrm{CH}_{3}\right), 5.03(s$, br., $1 \mathrm{H}, \mathrm{NH}) .{ }^{13} \mathrm{C} \mathrm{NMR}\left(100 \mathrm{MHz}, \mathrm{CDCl}_{3}\right) \delta 27.48\left(\mathrm{CH}_{3}\right), 41.64\left(\mathrm{CH}_{2}\right), 52.02\left(\mathrm{CH}_{3}\right), 60.58\left(\mathrm{CH}_{2}\right)$, 73.05 (C), 157.09 (C). HRMS (ESI) calculated for $\mathrm{C}_{8} \mathrm{H}_{17} \mathrm{NO}_{3} \mathrm{Na}\left([\mathrm{M}+\mathrm{Na}]^{+}\right)$: 198.1101. Found: 198.1093.

(2-Butoxyethyl)carbamic acid methyl ester (28): Prepared according to the general procedure (4) using reagent $8 \mathbf{a}(0.15 \mathrm{~g}, 0.62 \mathrm{mmol})$, 1-vinyloxy-butane $(0.19 \mathrm{mg}, 1.9 \mathrm{mmol})$, DTBP (46 mg, 0.31 $\mathrm{mmol})$ and thiophenol $(14 \mathrm{mg}, 0.12 \mathrm{mmol})$ in dry benzene $(1.5 \mathrm{ml})$. Purification by FC (pentane / tertbutylmethylether 6:1) provided 28 as a colourless oil (56 mg, $51 \%$ ). IR (neat) 3340br.m, 2958s, 2870s, 
$1713 s, 1537 s, 1464 m, 1370 w, 1260 s, 1194 w, 1118 s, 1030 w, 779 s \mathrm{~cm}^{-1} .{ }^{1} \mathrm{H}$ NMR $\left(400 \mathrm{MHz}, \mathrm{CDCl}_{3}\right) \delta$ $0.91\left(t, 3 \mathrm{H}, J=7.3 \mathrm{~Hz}, \mathrm{CH}_{3}\right), 1.41-1.30\left(m, 2 \mathrm{H}, \mathrm{CH}_{2}\right), 1.57-1.50\left(m, 2 \mathrm{H}, \mathrm{CH}_{2}\right), 3.36-3.34(m, 2 \mathrm{H}$, $\left.\mathrm{NHCH}_{2}\right), 3.41\left(t, 2 \mathrm{H}, J=6.6 \mathrm{~Hz}, \mathrm{OCH}_{2}\right), 3.46\left(t, 2 \mathrm{H}, J=5.1 \mathrm{~Hz}, \mathrm{OCH}_{2}\right), 3.66\left(s, 3 \mathrm{H}, \mathrm{CO}_{2} \mathrm{CH}_{3}\right), 5.03(s$, br., $1 \mathrm{H}, \mathrm{NH}) .{ }^{13} \mathrm{C} \mathrm{NMR}\left(100 \mathrm{MHz}, \mathrm{CDCl}_{3}\right) \delta 13.85\left(\mathrm{CH}_{3}\right), 19.27\left(\mathrm{CH}_{2}\right), 31.69\left(\mathrm{CH}_{2}\right), 41.00\left(\mathrm{CH}_{2}\right)$, $52.03\left(\mathrm{CH}_{3}\right), 69.46\left(\mathrm{CH}_{2}\right), 70.96\left(\mathrm{CH}_{2}\right), 157.06$ (C). HRMS (ESI) calculated for $\mathrm{C}_{8} \mathrm{H}_{17} \mathrm{NO}_{3} \mathrm{Na}$ $\left([\mathrm{M}+\mathrm{Na}]^{+}\right)$: 198.1101. Found: 198.1103.

Tetrahydropyran-3-yl)carbamic acid methyl ester (29a): Prepared according to the general procedure (4) using reagent $8 \mathbf{a}(0.15 \mathrm{~g}, 0.62 \mathrm{mmol})$, 3,4-dihydro- $2 H$-pyran $(0.13 \mathrm{~g}, 1.6 \mathrm{mmol})$, DTBP (46 mg, 0.31 $\mathrm{mmol})$ and thiophenol $(14 \mathrm{mg}, 0.12 \mathrm{mmol})$ in dry benzene $(1.5 \mathrm{ml})$ Purification by FC (pentane / tertbutylmethylether 1:1) provided 29a as a low melting white solid with another regio isomer in 1:4 ratio (ratio was determined by ${ }^{1} \mathrm{H}$ NMR spectroscopy) (60 mg, $60 \%$ (combined yield of both isomers)). Physical data of the major isomer is given. IR (neat) 3317br.m, 2949s, 2852m, 1711s, 1536s, 1454w, $1307 m, 1243 \mathrm{~s}, 1093 \mathrm{~cm}^{-1} .{ }^{1} \mathrm{H}$ NMR (400 MHz, $\left.\mathrm{CDCl}_{3}\right) \delta 1.60-1.51\left(\mathrm{~m}, 2 \mathrm{H}, \mathrm{CH}_{2}\right), 1.77-1.68(\mathrm{~m}, 1 \mathrm{H}$, $\mathrm{CHH}), 1.89-1.83(\mathrm{~m}, 1 \mathrm{H}, \mathrm{CH} H), 3.40-3.38(\mathrm{~m}, 1 \mathrm{H}, \mathrm{NHCH}), 3.78-3.60\left(\mathrm{~m}, 7 \mathrm{H}, \mathrm{CO}_{2} \mathrm{CH}_{3}, \mathrm{OCH}_{2}\right), 5.03(s$, br., 1H, NH). ${ }^{13} \mathrm{C}$ NMR $\left(100 \mathrm{MHz}, \mathrm{CDCl}_{3}\right) \delta 23.09\left(\mathrm{CH}_{2}\right), 28.85\left(\mathrm{CH}_{2}\right), 46.45(\mathrm{CH}), 51.93\left(\mathrm{CH}_{3}\right), 68.02$ $\left(\mathrm{CH}_{2}\right), 71.41\left(\mathrm{CH}_{2}\right), 156.29(\mathrm{C})$. HRMS (ESI) calculated for $\mathrm{C}_{7} \mathrm{H}_{13} \mathrm{NO}_{3} \mathrm{Na}\left([\mathrm{M}+\mathrm{Na}]^{+}\right)$: 182.0788 . Found: 182.0784 .

[2-(Acetylmethylamino)ethyl]carbamic acid methyl ester (30): Prepared according to the general procedure (4) using reagent $8 \mathbf{a}(0.15 \mathrm{~g}, 0.62 \mathrm{mmol}), N$-methyl- $N$-vinyl-acetamide $(0.16 \mathrm{~g}, 1.6 \mathrm{mmol})$, DTBP (46 mg, $0.31 \mathrm{mmol})$ and thiophenol $(10 \mathrm{mg}, 0.09 \mathrm{mmol})$ in dry benzene $(1.5 \mathrm{ml})$. Purification by FC (pentane / acetone 1:1) provided 30 as a colourless oil (60 mg, $55 \%$ ). IR (neat) $3321 \mathrm{br} . \mathrm{m}, 2949 w$, $1710 s, 1627 s, 1538 m, 1269 s, 1148 w, 1019 m, 779 w \mathrm{~cm}^{-1} .{ }^{1} \mathrm{H} \mathrm{NMR}\left(300 \mathrm{MHz}, \mathrm{CDCl}_{3}\right) \delta 2.02(s, 3 \mathrm{H}$, $\mathrm{COCH}_{3}$, rotamer-1), $2.03\left(s, 3 \mathrm{H}, \mathrm{COCH}_{3}\right.$, rotamer-2), $2.87\left(s, 3 \mathrm{H}, \mathrm{NCH}_{3}\right.$, rotamer-2), $2.98(s, 3 \mathrm{H}$, $\mathrm{NCH}_{3}$, rotamer-1), 3.32-3.21 ( $\left.m, 2 \mathrm{H}, \mathrm{CH}_{2}\right), 3.45-3.36\left(m, 2 \mathrm{H}, \mathrm{CH}_{2}\right), 3.57\left(s, 3 \mathrm{H}, \mathrm{CO}_{2} \mathrm{CH}_{3}\right.$, rotamer-1), $3.60\left(s, 3 \mathrm{H}, \mathrm{CO}_{2} \mathrm{CH}_{3}\right.$, rotamer-2), $5.51(s$, br., $1 \mathrm{H}, \mathrm{NH}$, rotamer-1), $5.74(s$, br., $1 \mathrm{H}, \mathrm{NH}$, rotamer-2). ${ }^{13}$ C.NMR $\left(75 \mathrm{MHz}, \mathrm{CDCl}_{3}\right) \delta 20.96\left(\mathrm{CH}_{3}\right.$, rotamer-2), $21.63\left(\mathrm{CH}_{3}\right.$, rotamer-1), $33.16\left(\mathrm{CH}_{3}\right.$, rotamer-2), $36.61\left(\mathrm{CH}_{3}\right.$, rotamer-1), $38.84\left(\mathrm{CH}_{2}\right.$, rotamer-2), $39.16\left(\mathrm{CH}_{2}\right.$, rotamer-1), $46.97\left(\mathrm{CH}_{2}\right.$, rotamer-1), 49.92 $\left(\mathrm{CH}_{2}\right.$, rotamer-2), $51.88\left(\mathrm{CH}_{3}\right.$, rotamer-1), $52.07\left(\mathrm{CH}_{3}\right.$, rotamer-2), $157.18(\mathrm{C}$, rotamer-2), $157.26(\mathrm{C}$, rotamer-1), $170.93\left(\mathrm{C}\right.$, rotamer-2), 171.69 (C, rotamer-1). HRMS (ESI) calculated for $\mathrm{C}_{7} \mathrm{H}_{14} \mathrm{~N}_{2} \mathrm{O}_{3} \mathrm{Na}$ $\left([\mathrm{M}+\mathrm{Na}]^{+}\right):$197.0897. Found: 197.0889.

[2-(2-Oxopyrrolidin-1-yl)-ethyl]carbamic acid methyl ester (31): Prepared according to the general procedure (4) using reagent $\mathbf{8 a}(0.15 \mathrm{~g}, 0.62 \mathrm{mmol})$, 1-vinyl-pyrrolidin-2-one $(0.17 \mathrm{~g}, 1.6 \mathrm{mmol})$, DTBP (46 mg, $0.31 \mathrm{mmol})$ and thiophenol $(10 \mathrm{mg}, 0.09 \mathrm{mmol})$ in dry benzene $(1.5 \mathrm{ml})$. Purification by FC (pentane / acetone 1:1) provided 31 as a low melting solid (64 mg, $55 \%$ ). IR (neat) 3322br.m, 2952m, $1705 s, 1666 s, 1538 s, 1466 m, 1267 s, 1195 w, 1148 w, 1023 m, 779 w \mathrm{~cm}^{-1} .{ }^{1} \mathrm{H} \mathrm{NMR}\left(400 \mathrm{MHz}, \mathrm{CDCl}_{3}\right) \delta$ 2.04-1.97 (m, 2H, $\left.\mathrm{CH}_{2}\right), 2.35\left(t, 2 \mathrm{H}, J=8.1 \mathrm{~Hz}, \mathrm{COCH}_{2}\right), 3.39-3.32\left(m, 4 \mathrm{H}, \mathrm{NCH}_{2} \mathrm{CH}_{2} \mathrm{NH}\right), 3.43(t, 2 \mathrm{H}$, $\left.J=7.2 \mathrm{~Hz}, \mathrm{NCH}_{2}\right), 3.62\left(s, 3 \mathrm{H}, \mathrm{CO}_{2} \mathrm{CH}_{3}\right), 5.29(s$, br., $1 \mathrm{H}, \mathrm{NH}) .{ }^{13} \mathrm{C} \mathrm{NMR}\left(100 \mathrm{MHz}, \mathrm{CDCl}_{3}\right) \delta 18.02$ $\left(\mathrm{CH}_{2}\right), 30.80\left(\mathrm{CH}_{2}\right), 39.11\left(\mathrm{CH}_{2}\right), 42.46\left(\mathrm{CH}_{2}\right), 47.64\left(\mathrm{CH}_{2}\right), 52.03\left(\mathrm{CH}_{3}\right), 157.24(\mathrm{C}), 176.04(\mathrm{C})$. HRMS (ESI) calculated for $\mathrm{C}_{8} \mathrm{H}_{14} \mathrm{~N}_{2} \mathrm{O}_{3} \mathrm{Na}\left([\mathrm{M}+\mathrm{Na}]^{+}\right)$: 209.0897. Found: 209.0896.

[2-(2-Oxoperhydroazepin-1-yl)ethyl]carbamic acid methyl ester (32): Prepared according to the general procedure (4) using reagent $8 \mathbf{a}(0.15 \mathrm{~g}, 0.62 \mathrm{mmol}), 1$-vinyl-perhydro-azepin-2-one $(0.26 \mathrm{~g}, 1.9$ $\mathrm{mmol})$, DTBP $(46 \mathrm{mg}, 0.31 \mathrm{mmol})$ and thiophenol $(14 \mathrm{mg}, 0.12 \mathrm{mmol})$ in dry benzene $(1.5 \mathrm{ml})$. Purification by FC (pentane / acetone 1:1) provided 32 as a colourless oil (69 $\mathrm{mg}, 52 \%$ yield). IR (neat) 3318br.m, 2932s, 1713s, 1629s, 1536m, 1355w, 1260s, 1197s, 1147m, 1083w, 1008m, 979m, 921m, $779 m, 730 s \mathrm{~cm}^{-1} .{ }^{1} \mathrm{H}$ NMR $\left(300 \mathrm{MHz}, \mathrm{CDCl}_{3}\right) \delta 1.73-1.56\left(\mathrm{~m}, 6 \mathrm{H}, \mathrm{CH}_{2}\right), 2.50-2.47\left(\mathrm{~m}, 2 \mathrm{H}, \mathrm{COCH}_{2}\right)$, 3.36-3.21 ( $\left.m, 4 \mathrm{H}, \mathrm{NCH}_{2} \mathrm{CH}_{2} \mathrm{NH}\right), 3.47\left(t, 2 \mathrm{H}, J=6.1 \mathrm{~Hz}, \mathrm{NCH}_{2}\right), 3.60\left(s, 3 \mathrm{H}, \mathrm{CO}_{2} \mathrm{CH}_{3}\right), 5.43(s$, br., $1 \mathrm{H}, \mathrm{NH}) .{ }^{13} \mathrm{C} \mathrm{NMR}\left(75 \mathrm{MHz}, \mathrm{CDCl}_{3}\right) \delta 23.24\left(\mathrm{CH}_{2}\right), 28.39\left(\mathrm{CH}_{2}\right), 29.80\left(\mathrm{CH}_{2}\right), 36.97\left(\mathrm{CH}_{2}\right), 40.02$ 
$\left(\mathrm{CH}_{2}\right), 47.69\left(\mathrm{CH}_{2}\right), 50.25\left(\mathrm{CH}_{2}\right), 51.94\left(\mathrm{CH}_{3}\right), 157.27$ (C), 177.00 (C). HRMS (ESI) calculated for $\mathrm{C}_{10} \mathrm{H}_{18} \mathrm{~N}_{2} \mathrm{O}_{3} \mathrm{Na}\left([\mathrm{M}+\mathrm{Na}]^{+}\right): 237.1210$. Found: 237.1211 .

[2-(1,3-Dioxo-1,3-dihydroisoindol-2-yl)ethyl]carbamic acid methyl ester (33): Prepared according to the general procedure (4) using reagent $8 \mathbf{a}(0.15 \mathrm{~g}, 0.62 \mathrm{mmol}), 2$-vinyl-isoindole-1,3-dione $(0.22 \mathrm{~g}, 1.6$ $\mathrm{mmol}$ ), DTBP (46 mg, $0.31 \mathrm{mmol})$ and thiophenol $(14 \mathrm{mg}, 0.12 \mathrm{mmol})$ in dry benzene $(1.5 \mathrm{ml})$. Purification by FC (pentane / tert-butylmethylether 1:1) provided 33 as a white solid (78 $\mathrm{mg}, 50 \%$ ). IR (neat) 3375 br $m, 2949 m, 1772 s, 1708 s, 1531 s, 1434 m, 1396 s, 1254 s, 1191 m, 1009 s, 721 s \mathrm{~cm}^{-1}$. ${ }^{1} \mathrm{H}$ NMR $\left(300 \mathrm{MHz}, \mathrm{CDCl}_{3}\right) \delta$ 3.52-3.40 (m, 2H, $\left.\mathrm{CH}_{2}\right), 3.59\left(s, 3 \mathrm{H}, \mathrm{CO}_{2} \mathrm{CH}_{3}\right), 3.88-3.78\left(m, 2 \mathrm{H}, \mathrm{CH}_{2}\right), 5.14(s$, br., $1 \mathrm{H}, \mathrm{NH}), 7.73-7.67$ ( $m, 2 \mathrm{H}, \mathrm{CH}$-aromatic), 7.86-7.78 (m, 2H, $\mathrm{CH}$-aromatic). ${ }^{13} \mathrm{C}$ NMR (75 MHz, $\left.\mathrm{CDCl}_{3}\right), 37.71\left(\mathrm{CH}_{2}\right), 40.27\left(\mathrm{CH}_{2}\right), 52.15\left(\mathrm{CH}_{3}\right), 123.32(\mathrm{CH}), 131.84(\mathrm{C}), 134.04(\mathrm{CH}), 157.02(\mathrm{C})$, 168.42 (C). HRMS (ESI) calculated for $\mathrm{C}_{12} \mathrm{H}_{12} \mathrm{~N}_{2} \mathrm{O}_{4} \mathrm{Na}\left([\mathrm{M}+\mathrm{Na}]^{+}\right)$: 271.0689. Found: 271.0685.

\section{References.}

a. Ohta, S.; Sumino, M.; Sasaki, T.; Yamagami, N. Heterocycles 1989, 29, 1455.

b. Kemper, J.; Studer, A. Angew. Chem. Int. Ed. 2005, 44, 4914.

c. Berson, J. A.; Ben-Efraim, D. A. J. Am. Chem. Soc. 1959, 81, 4094.

d. Newton, M. G; Pantaleo, N. S.; Kirbawy, S.; Allinger, N. L. J. Am. Chem. Soc 1978, 100, 2176. 
Table S1: Optimized ground state and transition structures of the fragmentation reaction of 34a-e and 35a-e (PBE/TZVPP): Electronic energies in a.u. and cartesian coordinates in a.u. (bohr).

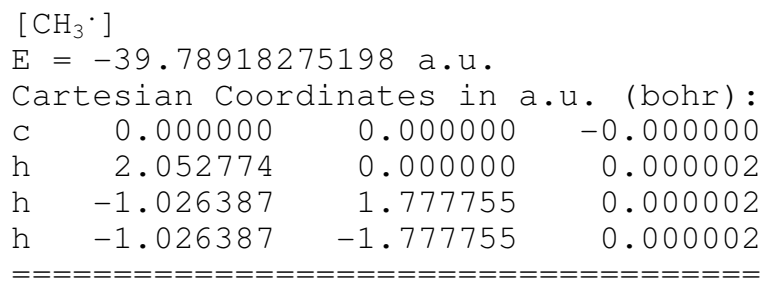

[34a] $\mathrm{X}=\mathrm{CH}_{3}$

$\mathrm{E}=-311.1062877810 \mathrm{a} \cdot \mathrm{u}$.

Cartesian Coordinates in a.u. (bohr): c $\quad 0.012643 \quad-0.004153 \quad 2.353521$

$\begin{array}{llll}\text { C } & 2.597811 & -0.001371 & 2.310002\end{array}$

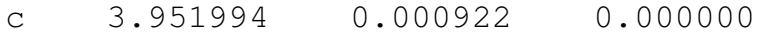

$\begin{array}{llll}\text { c } & 2.597811 & -0.001371 & -2.310002\end{array}$

c $\quad 0.012643 \quad-0.004153 \quad-2.353521$

C $\quad-1.590911 \quad-0.000441 \quad 0.000000$

$\begin{array}{llll}\text { C } & -3.326841 & -2.370128 & 0.000000\end{array}$

h $\quad-0.995080 \quad-0.007618 \quad 4.157086$

h $\quad 3.642239 \quad-0.003755 \quad 4.090057$

h $\quad 6.010717 \quad 0.002613 \quad 0.000000$

h $\quad 3.642239 \quad-0.003755 \quad-4.090057$

h $\quad-0.995080 \quad-0.007618 \quad-4.157086$

h $\quad-2.199895 \quad-4.111327 \quad 0.000000$

h $\quad-4.545584 \quad-2.374386 \quad-1.684314$

$\begin{array}{llll}\text { h } & -4.545584 & -2.374386 & 1.684314\end{array}$

$\begin{array}{llll}\text { C } & -3.313135 & 2.379397 & 0.000000\end{array}$

h $\quad-4.531698 \quad 2.390930 \quad 1.684428$

h $\quad-4.531698 \quad 2.390930 \quad-1.684428$

h $\quad-2.175785 \quad 4.113836 \quad 0.000000$

[34a-TS ] X $=\mathrm{CH}_{3}$

$\mathrm{E}=-311.0731823859 \mathrm{a} \cdot \mathrm{u}$.

Cartesian Coordinates in a.u. (bohr):

$\begin{array}{llll}\text { C } & 0.075470 & -1.047530 & 0.997799\end{array}$

$\begin{array}{llll}\text { C } & 1.894349 & -3.007258 & 0.639734\end{array}$

C $\quad 4.237732-2.508778-0.424966$

$\begin{array}{llll}\text { C } & 4.931537 & -0.027167 & -1.046197\end{array}$

$\begin{array}{llll}\text { C } & 3.266365 & 1.961720 & -0.501034\end{array}$

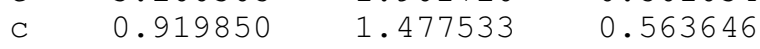

$\begin{array}{llll}\text { c } & -2.098846 & -1.583149 & -2.597272\end{array}$

C $\quad-2.147337-1.500842 \quad 2.744035$

$\begin{array}{llll}\text { h } & 1.381233 & -4.941262 & 1.146233\end{array}$

h $\quad 5.560329-4.056934 \quad-0.753115$

$\begin{array}{llll}\text { h } & 6.775023 & 0.359118 & -1.881283\end{array}$

h $\quad 3.829931 \quad 3.907478 \quad-0.888605$

h $\quad-0.352846 \quad 3.039835 \quad 1.010616$

h $\quad-0.585897 \quad-1.268091 \quad-3.954074$

h $\quad-3.522294-0.103505 \quad-2.437706$

h $\quad-2.764777 \quad-3.520442 \quad-2.386376$

h $\quad-2.946024 \quad-3.398242 \quad 2.489400$

h $\quad-3.658172 \quad-0.113401 \quad 2.435242$

h $\quad-1.535954-1.335447 \quad 4.726591$

$\left[\mathrm{SiH}_{3} \cdot\right] \quad \mathrm{X}=\mathrm{SiH}_{3}$

$\mathrm{E}=-291.0688264893 \mathrm{a} \cdot \mathrm{u}$.

Cartesian Coordinates in a.u. (bohr):

si $0.000000 \quad 0.000000-0.000002$ 


$\begin{array}{lrrr}\text { h } & 2.802970 & 0.000000 & 0.000014 \\ \mathrm{~h} & -1.401485 & 2.427443 & 0.000014 \\ \mathrm{~h} & -1.401485 & -2.427443 & 0.000014 \\ \mathrm{=}=============================\end{array}$

[34b] $\mathrm{X}=\mathrm{SiH}_{3}$

$\mathrm{E}=-562.3785829147 \mathrm{a} \cdot \mathrm{u}$.

Cartesian Coordinates in a.u. (bohr):

$\begin{array}{lrrr}\mathrm{C} & -0.592490 & -0.630324 & -2.360892 \\ \mathrm{C} & -3.035556 & 0.240151 & -2.300776 \\ \mathrm{C} & -4.284667 & 0.731963 & 0.012497 \\ \mathrm{C} & -3.038487 & 0.151998 & 2.306654 \\ \mathrm{C} & -0.596371 & -0.722858 & 2.335528 \\ \mathrm{C} & 0.948768 & -0.944029 & -0.017037 \\ \mathrm{C} & 2.668149 & -3.298727 & -0.061713 \\ \mathrm{~h} & 0.320100 & -1.050203 & -4.164115 \\ \mathrm{~h} & -4.050116 & 0.516941 & -4.076187 \\ \mathrm{~h} & -6.221215 & 1.428220 & 0.024625 \\ \mathrm{~h} & -4.056139 & 0.357112 & 4.089977 \\ \mathrm{~h} & 0.313442 & -1.214489 & 4.121944 \\ \mathrm{~h} & 1.508181 & -5.024536 & -0.099059 \\ \mathrm{~h} & 3.883822 & -3.376873 & 1.621149 \\ \mathrm{~h} & 3.890863 & -3.309453 & -1.741335 \\ \mathrm{si} & 3.149391 & 2.031325 & 0.038996 \\ \mathrm{~h} & 4.781851 & 2.048496 & -2.282803 \\ \mathrm{~h} & 4.812165 & 1.942750 & 2.337980 \\ \mathrm{~h} & 1.565724 & 4.368421 & 0.102920 \\ =====================================\end{array}$

[34b-TS $] \quad X=\mathrm{SiH}_{3}$

$E=-562.3644689436$ a.u.

Cartesian Coordinates in a.u. (bohr):

$\begin{array}{llll}\text { c } & -0.463525 & -0.768301 & -2.319353\end{array}$

C $\quad-2.806970 \quad 0.409694 \quad-2.283398$

$\begin{array}{llll}\text { C } & -3.987607 & 0.992547 & 0.016765\end{array}$

$\begin{array}{lrrr}\text { C } & -2.816765 & 0.292688 & 2.289355\end{array}$

C $\quad-0.473327 \quad-0.885275 \quad 2.275209$

C $\quad 0.870279 \quad-1.294660 \quad-0.031133$

C $\quad 3.066628 \quad-3.134918 \quad-0.072987$

h $\quad 0.428617-1.240559-4.119099$

h $\quad-3.747065 \quad 0.860003 \quad-4.062683$

h $\quad-5.825785 \quad 1.921534 \quad 0.036556$

h $\quad-3.764749 \quad 0.651536 \quad 4.085186$

h $\quad 0.411318 \quad-1.448597 \quad 4.052329$

h $\quad 2.337388 \quad-5.086475 \quad-0.123428$

h $\quad 4.256497 \quad-2.950479 \quad 1.615969$

h $\quad 4.263294 \quad-2.866166 \quad-1.745780$

si $\quad 3.378184 \quad 2.778825 \quad 0.058391$

$\begin{array}{llll}\text { h } & 4.936045 & 3.243896 & -2.275297\end{array}$

h $\quad 4.989657 \quad 3.167928 \quad 2.370217$

h $\quad 1.191490 \quad 4.571928 \quad 0.113656$

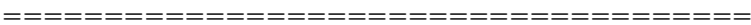


$\left[\mathrm{NH}_{2} \cdot\right] \quad \mathrm{X}=\mathrm{NH}_{2}$

$\mathrm{E}=-55.83100569658 \mathrm{a} \cdot \mathrm{u}$

Cartesian Coordinates in a.u. (bohr):

$\begin{array}{lrrr}\mathrm{n} & 0.000000 & 0.000000 & 0.155248 \\ \mathrm{~h} & 1.523510 & 0.000000 & -1.078657 \\ \mathrm{~h} & -1.523510 & 0.000000 & -1.078657\end{array}$

$=======$

[34c] $\mathrm{X}=\mathrm{NH}_{2}$

$\mathrm{E}=-327.1485060255 \mathrm{a} \cdot \mathrm{u}$.

Cartesian Coordinates in a.u. (bohr):

$\begin{array}{llll}\text { c } & -0.030921 & -0.073887 & -2.354191\end{array}$

$\begin{array}{llll}\text { c } & -2.620411 & -0.033232 & -2.317154\end{array}$

$\begin{array}{llll}\text { C } & -3.963397 & -0.024611 & -0.004398\end{array}$

c $\quad \begin{array}{lll}-2.609481 & -0.026642 & 2.308299\end{array}$

$\begin{array}{llll}\text { c } & -0.025181 & -0.022053 & 2.357962\end{array}$

$\begin{array}{llll}\text { C } & 1.571407 & -0.016958 & 0.000243\end{array}$

$\begin{array}{llll}\mathrm{n} & 3.187246 & 2.306685 & 0.084404\end{array}$

$\begin{array}{llll}\text { C } & 3.404099 & -2.287912 & 0.033053\end{array}$

h $\quad 0.988948 \quad-0.098088 \quad-4.151725$

$\begin{array}{lll}-3.668397 & -0.009470 & -4.094736\end{array}$

$\begin{array}{lll}-6.022427 & -0.000253 & 0.000634\end{array}$

$\begin{array}{lll}-3.657255 & -0.018289 & 4.086064\end{array}$

$\begin{array}{lll}1.004765 & 0.028899 & 4.144770\end{array}$

$\begin{array}{lll}2.046316 & 3.859160 & -0.085445\end{array}$

$\begin{array}{lll}4.352908 & 2.321134 & -1.462561\end{array}$

$\begin{array}{lll}2.359514 & -4.078316 & 0.056348\end{array}$

$\begin{array}{lll}4.622103 & -2.182738 & 1.711087\end{array}$

$4.612097-2.260388 \quad-1.661079$

[34c-TS ] X $=\mathrm{NH}_{2}$

$\mathrm{E}=-327.1246566284 \mathrm{a} \cdot \mathrm{u}$.

Cartesian Coordinates in a.u. (bohr):

$\begin{array}{llll}\text { c } & -0.325468 & -0.412688 & -2.216197\end{array}$

$\begin{array}{llll}\text { C } & -2.866339 & 0.256457 & -2.261819\end{array}$

C $\quad-4.205608 \quad 0.563831 \quad-0.003098$

$\begin{array}{lrrr}\text { c } & -2.971977 & 0.154429 & 2.313206\end{array}$

C $\quad-0.443231 \quad-0.516056 \quad 2.375574$

$\begin{array}{llll}\text { c } & 1.020711 & -0.697643 & 0.108726\end{array}$

$\begin{array}{llll}\text { n } & 2.738835 & 2.901499 & 0.147395\end{array}$

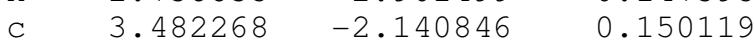

h $\quad 0.692676 \quad-0.717269-3.986266$

h $\quad-3.823072 \quad 0.510892 \quad-4.070555$

h $-6.200708-1.077475-0.038531$

h $\quad-4.024114 \quad 0.332883 \quad 4.077891$

h $\quad 0.496823 \quad-0.856365 \quad 4.179601$

h $\quad \begin{array}{lll}1.145927 & 3.964805 & 0.527075\end{array}$

h $2.955871 \quad 3.142898 \quad-1.780006$

h $\quad 3.104466 \quad-4.187359 \quad 0.150436$

h $\quad 4.582471 \quad-1.671118 \quad 1.840723$

h $\quad 4.640469-1.705825 \quad-1.514275$ 
$\left[\cdot \mathrm{NH}(\mathrm{CO}) \mathrm{CH}_{3}\right] \quad \mathrm{X}=\mathrm{NHAC}$

$\mathrm{E}=-208.3809462348 \mathrm{a} \cdot \mathrm{u}$.

Cartesian Coordinates in a.u. (bohr):

$\begin{array}{lrrr}\mathrm{n} & 1.518971 & 2.036848 & -0.068445 \\ \mathrm{C} & 0.046249 & -0.006966 & 0.076654 \\ \mathrm{~h} & 3.331860 & 1.728394 & 0.560335 \\ \mathrm{O} & 1.163224 & -2.107515 & -0.029107 \\ \mathrm{C} & -2.771536 & 0.226900 & 0.002667 \\ \mathrm{~h} & -3.346455 & 2.163608 & 0.463226 \\ \mathrm{~h} & -3.461663 & -0.253682 & -1.896327 \\ \mathrm{~h} & -3.620011 & -1.110682 & 1.340681\end{array}$

[34d] $\mathrm{X}=\mathrm{NHAC}$

$\mathrm{E}=-479.6892744028 \mathrm{a} \cdot \mathrm{u}$.

Cartesian Coordinates in a.u. (bohr):

$\begin{array}{llll}\text { C } & 4.802671 & -1.297318 & -1.470744\end{array}$

$\begin{array}{llll}\text { c } & 2.486388 & -2.247899 & -0.832481\end{array}$

$\begin{array}{llll}\text { C } & 0.537129 & -0.761096 & 0.629503\end{array}$

$\begin{array}{llll}\text { C } & 1.469730 & 1.868675 & 1.235146\end{array}$

c $\quad 3.800339 \quad 2.741875 \quad 0.532023$

$\begin{array}{llll}\text { C } & 5.518538 & 1.196562 & -0.813530\end{array}$

C $\quad-0.049260 \quad-2.240601 \quad 3.083719$

$\begin{array}{llll}\mathrm{n} & -1.735887 & -0.636312 & -1.061257\end{array}$

$\begin{array}{llll}\text { c } & -4.167783 & 0.244647 & -0.735635\end{array}$

$\begin{array}{llll}0 & -5.553053 & 0.474241 & -2.589431\end{array}$

$\begin{array}{llll}\text { C } & -5.135659 & 0.831008 & 1.897823\end{array}$

h $\quad 0.175826 \quad 3.099427 \quad 2.267280$

$\begin{array}{llll}\text { h } & 4.341128 & 4.674928 & 1.008284\end{array}$

h $\quad 7.365549 \quad 1.923934 \quad-1.358261$

$\begin{array}{llll}\text { h } & 6.127135 & -2.486621 & -2.513407\end{array}$

h $\quad 1.951029-4.172898 \quad-1.349749$

h $\quad-1.308032 \quad-0.776994 \quad-2.933241$

$\begin{array}{llll}\text { h } & 1.713453 & -2.538838 & 4.134349\end{array}$

h $\quad-0.871317 \quad-4.090307 \quad 2.618823$

h $\quad-1.358694 \quad-1.214374 \quad 4.317715$

h $\quad-5.526550 \quad-0.929789 \quad 2.927369$

h $\quad-3.805860 \quad 1.948327 \quad 3.029388$

h $-6.907586 \quad 1.875701 \quad 1.686042$ 
[34d-TS ] $\mathrm{X}=\mathrm{NHAC}$

$\mathrm{E}=-479.6709995128 \mathrm{a} \cdot \mathrm{u}$.

Cartesian Coordinates in a.u. (bohr):

$\begin{array}{llll}\text { c } & -3.227053 & 1.305619 & -1.225780\end{array}$

C $\quad-1.035910 \quad 1.362317 \quad 0.389852$

$\begin{array}{llll}\text { C } & -0.784316 & -0.721901 & 2.119272\end{array}$

C $\quad-2.369941 \quad-2.799271 \quad 2.001904$

$\begin{array}{llll}\text { C } & -4.369838 & -2.867866 & 0.262779\end{array}$

C $\quad-4.796483-0.780679 \quad-1.324578$

$\begin{array}{lll}0.014628 & 3.912142 & 1.129236\end{array}$

$\begin{array}{lll}1.559572 & 0.446152 & -2.299333\end{array}$

$\begin{array}{lll}3.707255 & -0.954239 & -2.088079\end{array}$

$\begin{array}{lll}5.319149 & -0.697312 & 0.256450\end{array}$

$\begin{array}{lll}4.414377 & -2.289901 & -3.888101\end{array}$

$\begin{array}{lll}0.724371 & -0.654903 & 3.523065\end{array}$

$\begin{array}{lll}-2.082344 & -4.377817 & 3.296305\end{array}$

$\begin{array}{lll}-5.614752 & -4.506720 & 0.176140\end{array}$

$\begin{array}{lll}-6.403198 & -0.789911 & -2.616752\end{array}$

$\begin{array}{lll}-3.591034 & 2.940163 & -2.430827\end{array}$

$\begin{array}{lll}0.487030 & -0.202493 & -3.778971\end{array}$

$\begin{array}{lll}-1.238403 & 4.801173 & 2.532326\end{array}$

$\begin{array}{lll}0.150866 & 5.162018 & -0.517049\end{array}$

$\begin{array}{lll}1.897314 & 3.746023 & 1.979966\end{array}$

$\begin{array}{lll}7.304682 & -0.529524 & -0.313497\end{array}$

$\begin{array}{lll}4.788837 & 0.926529 & 1.425297\end{array}$

$5.145191-2.429600 \quad 1.390376$

$\left[\cdot \mathrm{NH}(\mathrm{CO}) \mathrm{OCH}_{3}\right] \quad \mathrm{X}=\mathrm{NHMOC}$

$\mathrm{E}=-283.5653042733 \mathrm{a} . \mathrm{u}$.

Cartesian Coordinates in a.u. (bohr):

$\begin{array}{llll}\mathrm{n} & 3.104683 & 1.293684 & -0.124433\end{array}$

$\begin{array}{llll}\text { C } & 0.909034 & -0.057438 & 0.055557\end{array}$

$\begin{array}{llll}\text { h } & 4.414108 & 0.570488 & 1.116473\end{array}$

$\begin{array}{llll}0 & 0.942003 & -2.390297 & -0.022870\end{array}$

o $\quad-1.189899 \quad 1.363600 \quad 0.035650$

$\begin{array}{llll}\text { C } & -3.533118 & -0.040980 & -0.014364\end{array}$

h $\quad-3.724764-1.054445 \quad-1.812760$

$\begin{array}{llll}\text { h } & -5.021985 & 1.378438 & 0.182749\end{array}$

h $-3.606002-1.402046 \quad 1.548945$ 
[34e] $\mathrm{X}=\mathrm{NHMOC}$

$\mathrm{E}=-554.8798220048 \mathrm{a} \cdot \mathrm{u}$.

Cartesian Coordinates in a.u. (bohr):

$\begin{array}{lrrr}\mathrm{C} & 5.686956 & 0.221034 & -1.475703 \\ \mathrm{C} & 3.439793 & -0.885160 & -2.109782 \\ \mathrm{C} & 1.199567 & -0.976784 & -0.346830 \\ \mathrm{C} & 1.726973 & 0.395716 & 2.100643 \\ \mathrm{C} & 4.005592 & 1.495235 & 2.634024 \\ \mathrm{C} & 6.032704 & 1.423895 & 0.890441 \\ \mathrm{C} & 0.562360 & -3.784798 & 0.177788 \\ \mathrm{~h} & -0.931998 & 0.256570 & -1.745510 \\ \mathrm{C} & -3.374577 & 0.711011 & -1.003688 \\ \mathrm{O} & -4.840880 & 2.056068 & -2.174464 \\ \mathrm{~h} & 0.175413 & 0.466480 & 3.454755 \\ \mathrm{~h} & 4.262682 & 2.459319 & 4.440104 \\ \mathrm{~h} & 7.834556 & 2.306608 & 1.351251 \\ \mathrm{~h} & 7.246507 & 0.179388 & -2.825686 \\ \mathrm{~h} & 3.194374 & -1.812498 & -3.937891 \\ \mathrm{~h} & -0.390924 & 1.445193 & -3.150972 \\ \mathrm{~h} & 2.194088 & -4.710371 & 1.061861 \\ \mathrm{~h} & 0.127703 & -4.751772 & -1.608082 \\ \mathrm{~h} & -1.068630 & -3.943735 & 1.442972 \\ \mathrm{O} & -3.998393 & -0.544696 & 1.168922 \\ \mathrm{C} & -6.580224 & -0.183113 & 1.945333 \\ \mathrm{~h} & -7.888928 & -0.862001 & 0.486744 \\ \mathrm{~h} & -6.965798 & 1.821796 & 2.310058 \\ \mathrm{~h} & -6.790505 & -1.290621 & 3.680275 \\ \mathrm{~h}===============================\end{array}$

$[34 \mathbf{e}-\mathrm{TS}] \quad \mathrm{X}=\mathrm{NHMOC}$

$\mathrm{E}=-554.8599261059 \mathrm{a} . \mathrm{u}$.

Cartesian Coordinates in a.u. (bohr):

$\begin{array}{llll}\text { c } & -3.242916 & 1.422516 & -1.064656\end{array}$

$\begin{array}{llll}\text { C } & -0.917365 & 1.208094 & 0.323427\end{array}$

C $\quad-0.619119 \quad-1.031368 \quad 1.828632$

$\begin{array}{llll}\text { C } & -2.363898 & -2.983370 & 1.737935\end{array}$

C $\quad-4.538487 \quad-2.768287 \quad 0.241033$

C $\quad-4.966641 \quad-0.538573 \quad-1.143594$

$\begin{array}{rrr}-4.966641 & -0.538573 & -1.143594 \\ 0.444299 & 3.597199 & 1.077424\end{array}$

$\begin{array}{lll}1.396102 & 0.213973 & -2.673435\end{array}$

$3.623968-1.058625-2.358081$

$4.440584-2.668782-3.824946$

$\begin{array}{lll}1.062473 & -1.189799 & 3.009576\end{array}$

$\begin{array}{lll}-2.050535 & -4.686975 & 2.855425\end{array}$

$\begin{array}{lll}-5.908066 & -4.305939 & 0.173134\end{array}$

$\begin{array}{lll}-6.690784 & -0.342134 & -2.257263\end{array}$

$\begin{array}{lll}-3.594789 & 3.162788 & -2.114020\end{array}$

$\begin{array}{lll}0.217927 & -0.866453 & -3.763865\end{array}$

$\begin{array}{lll}-0.533664 & 4.467618 & 2.694731\end{array}$

$\begin{array}{lll}0.460765 & 4.956952 & -0.486008\end{array}$

$\begin{array}{lll}2.399896 & 3.198362 & 1.626914\end{array}$

$\begin{array}{lll}4.973439 & -0.203047 & -0.326712\end{array}$

$\begin{array}{lll}7.428944 & -1.335576 & -0.058998\end{array}$

$8.639042-0.858754-1.675383$

$\begin{array}{lll}7.284355 & -3.401230 & 0.078176\end{array}$

$8.214757 \quad-0.546295 \quad 1.685140$ 
[toluene]

$\mathrm{E}=-271.2971380777 \mathrm{a} \cdot \mathrm{u}$.

Cartesian Coordinates in a.u. (bohr):

$\begin{array}{llll}\text { C } & -4.650094 & 0.003524 & 0.000000\end{array}$

$\begin{array}{lrrr}\text { h } & -5.410490 & 1.933233 & 0.000000\end{array}$

h $\quad-5.402813 \quad-0.978585 \quad 1.670842$

h $\quad-5.402813 \quad-0.978585-1.670842$

$\begin{array}{llll}\text { C } & -1.802104 & 0.008578 & 0.000000\end{array}$

c $\quad-0.444650 \quad-2.270659 \quad 0.000000$

$\begin{array}{llll}\text { C } & 2.191436 & -2.282766 & 0.000000\end{array}$

C $\quad 3.530236 \quad-0.005740 \quad 0.000000$

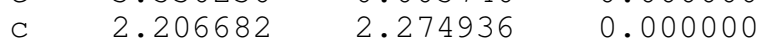

C $\quad-0.434324 \quad 2.275531 \quad 0.000000$

$\begin{array}{llll}\text { h } & -1.473298 & -4.060804 & 0.000000\end{array}$

h $\quad 3.207193 \quad-4.077350 \quad 0.000000$

$\begin{array}{llll}\text { h } & 5.591416 & -0.012514 & 0.000000\end{array}$

$\begin{array}{llll}\text { h } & 3.230478 & 4.064848 & 0.000000\end{array}$

h $\quad-1.455792 \quad 4.069192 \quad 0.000000$

$==\mathrm{=}=\mathrm{=}=\mathrm{=}=\mathrm{=}=\mathrm{=}=\mathrm{=}=\mathrm{=}=\mathrm{=}=\mathrm{=}=\mathrm{=}=\mathrm{=}=\mathrm{=}=\mathrm{x}$

[3-Methoxycarbonyl-5-methyl-toluene] $\mathrm{E}=-538.3010126599 \mathrm{a} \cdot \mathrm{u}$.

Cartesian Coordinates in a.u. (bohr):

c $\quad-5.187780 \quad-4.119079 \quad-0.007761$

h $\quad-6.626410 \quad-2.625827 \quad-0.001322$

h $\quad-5.508078 \quad-5.321957 \quad 1.657369$

h $\quad-5.506448 \quad-5.306172 \quad-1.684490$

C $\quad-2.557984 \quad-3.026028 \quad-0.001472$

C $\quad-0.437938-4.619454-0.013446$

c $\quad 2.034829-3.675283-0.012099$

C $\quad 2.373478 \quad-1.053467 \quad-0.011146$

$\begin{array}{llll}\text { C } & 0.292026 & 0.578100 & 0.000763\end{array}$

$\begin{array}{lrrr}\text { c } & -2.166570 & -0.414709 & 0.007793\end{array}$

h $\quad-0.726138 \quad-6.666370 \quad-0.030631$

h $\quad \begin{array}{rrrr}-263604 & -0.230284 & -0.026213\end{array}$

$\begin{array}{lrrr}\text { h } & -3.777878 & 0.867438 & 0.010767\end{array}$

$\begin{array}{llll}\text { C } & 4.273122 & -5.434894 & 0.031429\end{array}$

h $\quad 5.893146 \quad-4.623288-0.979637$

h $\quad 3.817182 \quad-7.267155 \quad-0.829523$

h $\quad 4.889922 \quad-5.804147 \quad 1.984514$

$\begin{array}{llll}\text { c } & 0.803677 & 3.348468 & -0.006601\end{array}$

o $\quad 2.901080 \quad 4.300256 \quad-0.031606$

$\begin{array}{llll}0 & -1.361662 & 4.741970 & 0.019961\end{array}$

$\begin{array}{llll}\text { c } & -0.975098 & 7.434854 & 0.015887\end{array}$

h $\quad-2.867133 \quad 8.266651 \quad 0.089080$

$\begin{array}{llll}\text { h } & 0.017061 & 8.027039 & -1.707211\end{array}$

h $\quad 0.145242 \quad 8.015200 \quad 1.662245$ 
[35a] $\mathrm{X}=\mathrm{CH}_{3}$

$\mathrm{E}=-578.1097454938$ a.u.

Cartesian Coordinates in a.u. (bohr):

\begin{tabular}{|c|c|c|c|}
\hline $\mathrm{C}$ & 0.223062 & 1.855451 & -0.001262 \\
\hline 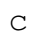 & -0.923903 & -0.473685 & 0.000183 \\
\hline & 0.528611 & -2.729782 & 0.000902 \\
\hline & 3.211115 & -2.621585 & -0.001262 \\
\hline & 4.396577 & -0.317174 & -0.002568 \\
\hline & 3.042672 & 2.184794 & 0.000217 \\
\hline & 3.831729 & 3.728606 & -2.372585 \\
\hline & -0.926311 & 3.566758 & -0.001901 \\
\hline & -3.734474 & -0.761507 & 0.000696 \\
\hline & -0.461165 & -4.535212 & 0.002250 \\
\hline & 4.695630 & -5.053264 & -0.002798 \\
\hline & 6.462894 & -0.236491 & -0.00424 \\
\hline & 3.310877 & 2.727746 & -4.112814 \\
\hline & 5.888178 & 4.032886 & -2.379298 \\
\hline & 2.898701 & 5.586117 & -2.374711 \\
\hline & 3.829383 & 3.721374 & 2.378757 \\
\hline & 2.894183 & 5.577777 & 2.387002 \\
\hline & 5.885496 & 4.027934 & 2.387024 \\
\hline & 3.309161 & 2.713809 & 4.115286 \\
\hline & 6.738488 & -4.696184 & -0.003248 \\
\hline & 4.239686 & -6.205036 & 1.66693 \\
\hline & 4.238703 & -6.203556 & -1.67328 \\
\hline & -4.961984 & 1.499534 & -0.00282 \\
\hline & -4.838840 & -2.782073 & 0.00395 \\
\hline & -7.676145 & 1.316096 & -0.00205 \\
\hline & -8.362511 & 3.267044 & -0.0059 \\
\hline & -8.341477 & 0.303223 & -1.6857 \\
\hline & -8.34092 & 0.310102 & 1.686 \\
\hline
\end{tabular}

[35a-TS ] X $=\mathrm{CH}_{3}$

$\mathrm{E}=-578.0769424239 \mathrm{a} \cdot \mathrm{u}$.

Cartesian Coordinates in a.u. (bohr):

$\begin{array}{llll}\text { C } & 0.051254 & -1.130847 & 0.853969\end{array}$

$\begin{array}{llll}\mathrm{C} & -1.813470 & -3.090213 & 0.315540\end{array}$

C $\quad 4.161790 \quad-2.533902-0.737379$

$\begin{array}{llll}\text { C } & 4.880830 & -0.008607 & -1.144920\end{array}$

$\begin{array}{llll}\text { C } & 4.880830 & -0.008607 & -1.144920 \\ \text { C } & 3.286997 & 1.984560 & -0.413379\end{array}$

$\begin{array}{lrrr}\text { C } & 0.945010 & 1.405334 & 0.634572\end{array}$

$\begin{array}{llll}\text { c } & -2.154034 & -1.375353 & -2.748225\end{array}$

C $\quad-2.164581 \quad-1.680138 \quad 2.582445$

$\begin{array}{lrrr}\mathrm{C} & -2.164581 & -1.680138 & 2.582445 \\ \mathrm{~h} & 1.284980 & -5.050470 & 0.660101\end{array}$

6.731540

0.361789

2.940585

$-0.658029$

$-0.991240$

$-1.972448$

1.242469

$-3.534969$

0.121935

$-2.865165$

$-3.306534$

$-2.987479$

$-3.544936$

$-0.256067$

$-1.535675$

4. 122399

$-1.662772$

4.686826

6.007936

5.177343

4.271095

5.995241

5.006479

6.010694

$-4.542235$

8.079911

$-4.154146$

5.134089

$-6.907943$

6.853088

$-8.916816$

7.278772

$-8.896805$

$5.881097-10.665403$

$-4.106321$

$-2.444830$

$-2.676214$

2.199727

2.385658

4.566060

$-0.747419$

0.129321

$-2.762975$

0.093182

$-1.441341$

$-2.375176$

$-0.925460$

$-1.564566$

$-3.594774$

$-1.040079$

8.629279

$-8.722745$

$-0.510486$ 
[35b] $\mathrm{X}=\mathrm{SiH}_{3}$

$\mathrm{E}=-829.3821310741 \mathrm{a} \cdot \mathrm{u}$.

Cartesian Coordinates in a.u. (bohr):

$\begin{array}{lrrr}\mathrm{C} & -1.144953 & -0.802418 & -3.933696 \\ \mathrm{C} & -3.241614 & -0.156366 & -2.536200 \\ \mathrm{C} & -2.995401 & 0.276680 & 0.098827 \\ \mathrm{C} & -0.643922 & -0.136016 & 1.319724 \\ \mathrm{C} & 1.479055 & -0.788554 & -0.039646 \\ \mathrm{C} & 1.457789 & -0.906382 & -2.846520 \\ \mathrm{C} & 3.089837 & -3.066376 & -3.931018 \\ \mathrm{~h} & -1.339175 & -1.184746 & -5.954484 \\ \mathrm{~h} & -4.622843 & 0.797142 & 1.246303 \\ \mathrm{~h} & 3.253697 & -1.163852 & 0.936937 \\ \mathrm{~h} & 2.277389 & -4.905192 & -3.399440 \\ \mathrm{~h} & 5.033003 & -2.984610 & -3.201655 \\ \mathrm{~h} & 3.174058 & -2.976344 & -6.004520 \\ \mathrm{Si} & 3.022006 & 2.286956 & -3.892015 \\ \mathrm{~h} & 3.056851 & 2.412357 & -6.726392 \\ \mathrm{~h} & 5.684989 & 2.420606 & -2.920580 \\ \mathrm{~h} & 1.518258 & 4.441411 & -2.858622 \\ \mathrm{C} & -5.808800 & 0.057083 & -3.747652 \\ \mathrm{~h} & -5.750841 & -0.461312 & -5.754620 \\ \mathrm{~h} & -6.530783 & 2.002345 & -3.604977 \\ \mathrm{~h} & -7.189100 & -1.169653 & -2.794091 \\ \mathrm{C} & -0.593255 & 0.113937 & 4.133098 \\ \mathrm{O} & -2.419189 & 0.659479 & 5.424643 \\ \mathrm{O} & 1.738391 & -0.331037 & 5.124985 \\ \mathrm{C} & 1.875709 & -0.124479 & 7.834461 \\ \mathrm{~h} & 0.565788 & -1.455467 & 8.737288 \\ \mathrm{~h} & 1.397057 & 1.799726 & 8.444529 \\ \mathrm{~h} & 3.832637 & -0.578395 & 8.325619 \\ ===================================\end{array}$

[35b-TS ] $\mathrm{X}=\mathrm{SiH}_{3}$

$\mathrm{E}=-829.3681024604 \mathrm{a} \cdot \mathrm{u}$.

Cartesian Coordinates in a.u. (bohr):

c $-0.474543 \quad-0.802647 \quad-2.186624$

$-2.797353$

$\begin{array}{llll}\text { C } & -3.950877 & 1.141505 & 0.012962\end{array}$

$\begin{array}{llll}\text { C } & -2.822888 & 0.526995 & 2.343887\end{array}$

C $\quad-0.496936 \quad-0.696847 \quad 2.408952$

$\begin{array}{llll}\text { C } & 0.835769 & -1.244077 & 0.131775\end{array}$

$\begin{array}{llll}\text { C } & 2.983272 & -3.142969 & 0.184656\end{array}$

h $\quad 0.418470 \quad-1.398321 \quad-3.950915$

$\begin{array}{lrrr}\text { h } & -5.771035 & 2.106706 & 0.029493\end{array}$

h $\quad 0.349873 \quad-1.193470 \quad 4.219187$

h $\quad 2.203349-\quad-5.074928-0.166363$

h $\quad 4.140239-2.942513 \quad 1.893966$

h $\quad 4.221189 \quad-2.950687 \quad-1.468693$

si $\quad 3.418733 \quad 2.727834 \quad-0.113044$

h $\quad 4.605143 \quad 2.562173 \quad-2.687247$

$\begin{array}{llll}\text { h } & 5.373191 & 3.574000 & 1.779173\end{array}$

$\begin{array}{lll}1.282695 & 4.581668 & -0.152798\end{array}$

$\begin{array}{lll}-4.052314 & 1.027560 & -4.755898\end{array}$

$\begin{array}{lll}-3.263772 & -0.120017 & -6.293398\end{array}$

$\begin{array}{lll}-3.782598 & 3.027181 & -5.263048\end{array}$

$\begin{array}{lll}-6.098583 & 0.690363 & -4.667683\end{array}$

$\begin{array}{lll}-4.230016 & 1.208886 & 4.691714\end{array}$

$\begin{array}{lll}-6.290194 & 2.235769 & 4.729055\end{array}$

$\begin{array}{lll}-2.950966 & 0.555038 & 6.826019\end{array}$

$\begin{array}{lll}-4.232839 & 1.165300 & 9.147872\end{array}$

$\begin{array}{lll}-6.061623 & 0.191786 & 9.249795\end{array}$

$\begin{array}{lll}-4.556513 & 3.209444 & 9.286477\end{array}$

$\begin{array}{lll}-2.976888 & 0.521012 & 10.659069\end{array}$ 
[35c] $\mathrm{X}=\mathrm{NH}_{2}$

$\mathrm{E}=-594.1507179010 \mathrm{a} \cdot \mathrm{u}$.

Cartesian Coordinates in a.u. (bohr):

$\begin{array}{lrrr}\mathrm{C} & -4.372653 & -0.344835 & -0.074983 \\ \mathrm{C} & -3.176204 & -2.658227 & -0.033967 \\ \mathrm{C} & -0.507568 & -2.740377 & -0.028794 \\ \mathrm{C} & 0.943341 & -0.473918 & -0.023307 \\ \mathrm{C} & -0.205908 & 1.850064 & -0.012757 \\ \mathrm{C} & -3.029895 & 2.160053 & -0.014255 \\ \mathrm{~h} & -3.701296 & 3.637025 & 2.303917 \\ \mathrm{C} & -3.833111 & 3.803579 & -2.290303 \\ \mathrm{~h} & -6.439786 & -0.257424 & -0.108789 \\ \mathrm{C} & -4.678551 & -5.086657 & 0.000559 \\ \mathrm{~h} & 0.495663 & -4.538321 & -0.013885 \\ \mathrm{C} & 3.752782 & -0.759802 & -0.008010 \\ \mathrm{~h} & 0.909820 & 3.579496 & 0.045056 \\ \mathrm{~h} & -3.388414 & 2.533613 & 3.860554 \\ \mathrm{~h} & -5.598058 & 4.025818 & 2.282335 \\ \mathrm{~h} & -3.315428 & 2.892289 & -4.078499 \\ \mathrm{~h} & -2.915415 & 5.662264 & -2.175843 \\ \mathrm{~h} & -5.895180 & 4.082043 & -2.275222 \\ \mathrm{~h} & -3.429976 & -6.742047 & -0.004407 \\ \mathrm{~h} & -5.883552 & -5.204264 & 1.689576 \\ \mathrm{~h} & -5.926266 & -5.225321 & -1.655215 \\ \mathrm{O} & 4.977392 & 1.500498 & -0.009914 \\ \mathrm{O} & 4.856543 & -2.781927 & 0.003201 \\ \mathrm{C} & 7.692229 & 1.320799 & 0.008944 \\ \mathrm{~h} & 8.374908 & 3.272867 & 0.008646 \\ \mathrm{~h} & 8.346994 & 0.317033 & 1.702072 \\ \mathrm{~h} & 8.369569 & 0.308313 & -1.670067 \\ \mathrm{I}=============================== \\ \mathrm{H} & & & \end{array}$

[35c-TS ] X $=\mathrm{NH}_{2}$

$\mathrm{E}=-594.1278797498 \mathrm{a} \cdot \mathrm{u}$.

Cartesian Coordinates in a.u. (bohr):

\begin{tabular}{|c|c|c|c|}
\hline $\mathrm{C}$ & -0.364597 & -0.497571 & -2.144194 \\
\hline C & -2.896651 & 0.233105 & -2.284124 \\
\hline C & -4.205309 & 0.680370 & -0.028790 \\
\hline & -3.003220 & 0.368768 & 2.327636 \\
\hline & -0.479926 & -0.351182 & 2.445721 \\
\hline & 0.972458 & -0.677671 & 0.193599 \\
\hline & 2.764040 & 2.868495 & 0.079818 \\
\hline & 3.399761 & -2.175811 & 0.313661 \\
\hline & 0.657431 & -0.936201 & -3.886494 \\
\hline & -6.190871 & 1.232202 & -0.045858 \\
\hline & 0.431246 & -0.619582 & 4.271617 \\
\hline & 1.204663 & 4.000707 & 0.394544 \\
\hline & 2.998223 & 2.999991 & -1.856518 \\
\hline & 2.973074 & -4.210345 & 0.403971 \\
\hline & 4.502144 & -1.655175 & 1.987370 \\
\hline & 4.574731 & -1.843485 & -1.362565 \\
\hline & -4.189037 & 0.542427 & -4.802876 \\
\hline & -3.328413 & -0.669918 & -6.250740 \\
\hline & -4.046618 & 2.506854 & -5.47529 \\
\hline & -6.207733 & 0.079687 & -4.67953 \\
\hline & -4.561672 & 0.794027 & 4.641565 \\
\hline & -6.783757 & 1.399464 & 4.62807 \\
\hline & -3.221306 & 0.431235 & 6.804783 \\
\hline & -4.638247 & 0.813274 & 9.096679 \\
\hline & -6.239942 & -0.501387 & 9.19224 \\
\hline & -5.362058 & 2.754805 & 9.1917 \\
\hline & -3.305676 & 0.454609 & 10.636660 \\
\hline
\end{tabular}

[35d] $\mathrm{X}=\mathrm{NHAC}$ 
$\mathrm{E}=-746.6919894066 \mathrm{a} \cdot \mathrm{u}$.

Cartesian Coordinates in a.u. (bohr):

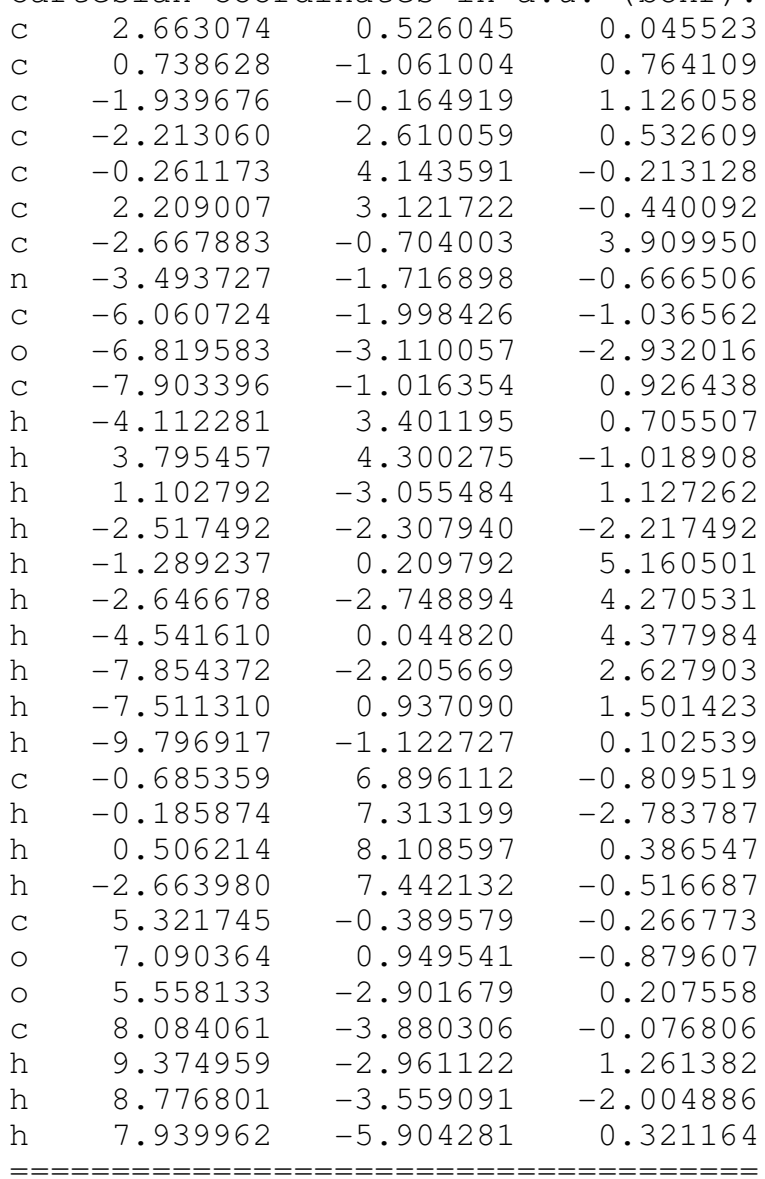

[35d-TS1b] $\mathrm{X}=\mathrm{NHAC}$

$\mathrm{E}=-746.6739213818 \mathrm{a} \cdot \mathrm{u}$.

Cartesian Coordinates in a.u. (bohr):

$\begin{array}{rrrr}\mathrm{C} & 2.200123 & 1.618782 & 0.132175 \\ \mathrm{C} & 0.768890 & 2.981684 & -1.594179 \\ \mathrm{C} & -1.805982 & 2.297656 & -2.126369 \\ \mathrm{C} & -2.666356 & -0.044254 & -1.046530 \\ \mathrm{C} & -1.246899 & -1.414732 & 0.682603 \\ \mathrm{C} & 1.191918 & -0.551307 & 1.294402 \\ \mathrm{C} & -2.928421 & 3.160451 & -4.607525 \\ \mathrm{C} & -2.262962 & -3.792048 & 1.873347 \\ \mathrm{C} & 4.851485 & 2.333062 & 0.797155 \\ \mathrm{O} & 5.603871 & 4.486911 & -0.380393 \\ \mathrm{C} & 8.148533 & 5.269872 & 0.195794 \\ \mathrm{O} & 6.200921 & 1.139251 & 2.229275 \\ \mathrm{~h} & -3.438166 & 4.920093 & 0.160717 \\ \mathrm{C} & -5.576050 & 4.728026 & 1.578761 \\ \mathrm{O} & -5.723377 & 5.864648 & 3.630502 \\ \mathrm{C} & -7.823725 & 3.309039 & 0.531870 \\ \mathrm{~h} & -4.531742 & -0.737311 & -1.594004 \\ \mathrm{~h} & 2.371918 & -1.574316 & 2.639387 \\ \mathrm{~h} & 1.573267 & 4.631818 & -2.526612 \\ \mathrm{~h} & -1.979996 & 5.598534 & 1.244348 \\ \mathrm{~h} & -2.156800 & 2.008720 & -6.158885 \\ \mathrm{~h} & -2.474519 & 5.147824 & -4.972951 \\ \mathrm{~h} & -4.990134 & 2.947533 & -4.626635 \\ \mathrm{~h} & -9.539485 & 4.410040 & 0.903731 \\ \mathrm{~h} & -7.662040 & 2.932284 & -1.497234 \\ \mathrm{~h} & -8.016303 & 1.502091 & 1.539449 \\ \mathrm{~h} & -4.084282 & -4.337191 & 1.045656 \\ \mathrm{~h} & -2.542630 & -3.533155 & 3.917378\end{array}$




$\begin{array}{lrrr}\text { h } & -0.940860 & -5.376688 & 1.636713 \\ \mathrm{~h} & 8.357043 & 5.623456 & 2.228582 \\ \mathrm{~h} & 8.448834 & 7.007643 & -0.883313 \\ \mathrm{~h} & 9.505751 & 3.808286 & -0.372499 \\ \mathrm{C}==============================\end{array}$

[35e] $\mathrm{X}=$ NHMOC

$E=-821.8825482138$ a.u.

Cartesian Coordinates in a.u. (bohr):

$\begin{array}{lrrr}\mathrm{C} & 3.313376 & -0.034857 & 0.511388 \\ \mathrm{C} & 1.403800 & -0.697504 & -1.117572 \\ \mathrm{C} & -1.280398 & -1.078705 & -0.255920 \\ \mathrm{C} & -1.603688 & -0.495709 & 2.515382 \\ \mathrm{C} & 0.335885 & 0.196486 & 4.088119 \\ \mathrm{C} & 2.825476 & 0.406127 & 3.108916 \\ \mathrm{C} & -2.011314 & -3.862267 & -0.806585 \\ \mathrm{~h} & -2.848818 & 0.666294 & -1.841291 \\ \mathrm{C} & -5.408305 & 1.090025 & -1.818890 \\ \mathrm{O} & -6.425823 & 2.835677 & -2.934464 \\ \mathrm{~h} & -3.523752 & -0.665299 & 3.249790 \\ \mathrm{~h} & 4.401645 & 0.939894 & 4.322020 \\ \mathrm{~h} & 1.794683 & -1.028693 & -3.113054 \\ \mathrm{~h} & -1.899211 & 2.163788 & -2.574618 \\ \mathrm{~h} & -0.742662 & -5.129609 & 0.234871 \\ \mathrm{~h} & -1.830088 & -4.248579 & -2.838699 \\ \mathrm{~h} & -3.962811 & -4.232982 & -0.221840 \\ \mathrm{O} & -6.693488 & -0.683511 & -0.446551 \\ \mathrm{C} & -9.393388 & -0.342012 & -0.462634 \\ \mathrm{~h} & -10.137418 & -0.462115 & -2.394540 \\ \mathrm{~h} & -9.906667 & 1.499980 & 0.339948 \\ \mathrm{~h} & -10.145805 & -1.877898 & 0.701435 \\ \mathrm{C} & -0.122130 & 0.754654 & 6.844001 \\ \mathrm{~h} & 0.374041 & 2.722218 & 7.296304 \\ \mathrm{~h} & 1.052232 & -0.460046 & 8.054829 \\ \mathrm{~h} & -2.108079 & 0.455633 & 7.358542 \\ \mathrm{C} & 5.987980 & 0.264442 & -0.359012 \\ \mathrm{O} & 7.743611 & 0.836469 & 1.015080 \\ \mathrm{O} & 6.258161 & -0.171692 & -2.875618 \\ \mathrm{C} & 8.801930 & 0.096750 & -3.809210 \\ \mathrm{~h} & 8.691977 & -0.304115 & -5.834657 \\ \mathrm{~h} & 10.069963 & -1.245306 & -2.864217 \\ \mathrm{~h} & 9.499935 & 2.021792 & -3.479519 \\ \mathrm{~h}===================================\end{array}$

[35e-TS $\quad \mathrm{X}=\mathrm{NHMOC}$

$\mathrm{E}=-821.8628398110 \mathrm{a} \cdot \mathrm{u}$.

Cartesian Coordinates in a.u. (bohr):

$\begin{array}{rrrr}\text { C } & -1.792363 & 1.923558 & -0.385970 \\ \mathrm{C} & 0.531341 & 1.740453 & 0.999036 \\ \mathrm{C} & 0.862949 & -0.483910 & 2.516163 \\ \mathrm{C} & -0.821794 & -2.500435 & 2.460458 \\ \mathrm{C} & -2.996693 & -2.292458 & 0.952490 \\ \mathrm{C} & -3.485508 & -0.079227 & -0.447002 \\ \mathrm{C} & 1.848784 & 4.154122 & 1.758817 \\ \mathrm{n} & 2.840885 & 0.818470 & -2.014866 \\ \mathrm{C} & 5.079974 & -0.435805 & -1.701384 \\ \mathrm{O} & 5.915507 & -2.026420 & -3.178632 \\ \mathrm{~h} & 2.546532 & -0.593675 & 3.703500 \\ \mathrm{~h} & -4.373852 & -3.823501 & 0.862235 \\ \mathrm{~h} & -2.190344 & 3.646783 & -1.439046 \\ \mathrm{~h} & 1.683816 & -0.258932 & -3.130642 \\ \mathrm{~h} & 0.861412 & 4.996187 & 3.385159 \\ \mathrm{~h} & 1.830755 & 5.520465 & 0.201671 \\ \mathrm{~h} & 3.814205 & 3.790441 & 2.297263 \\ \mathrm{O} & 6.413709 & 0.414901 & 0.341485 \\ \mathrm{C} & 8.880112 & -0.695439 & 0.607818\end{array}$




$\begin{array}{lrrr}\mathrm{h} & 10.089406 & -0.194573 & -1.001683 \\ \mathrm{~h} & 8.755369 & -2.763350 & 0.729330 \\ \mathrm{~h} & 9.653124 & 0.088857 & 2.359811 \\ \mathrm{C} & -0.340958 & -4.863735 & 3.972424 \\ \mathrm{~h} & 0.238409 & -6.428346 & 2.730697 \\ \mathrm{~h} & -2.051606 & -5.480415 & 4.974783 \\ \mathrm{~h} & 1.165738 & -4.576381 & 5.367982 \\ \mathrm{C} & -5.894251 & 0.016297 & -1.920220 \\ \mathrm{O} & -7.461210 & -1.669563 & -1.961828 \\ \mathrm{O} & -6.150738 & 2.211429 & -3.226211 \\ \mathrm{C} & -8.445371 & 2.409548 & -4.680221 \\ \mathrm{~h} & -8.359751 & 4.260775 & -5.596829 \\ \mathrm{~h} & -10.104599 & 2.278711 & -3.442752 \\ \mathrm{~h} & -8.542991 & 0.895170 & -6.093835 \\ =====================================\end{array}$


ฐ

욤

웅

カヤトてて

209' 2

$909^{\circ} \mathrm{C}$

0

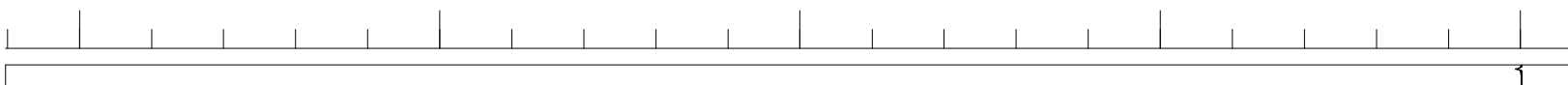



6ฤt๑9

५ะ6.9

$800^{\circ} \mathrm{L}$

$99 \varepsilon^{\circ} L$

$\nabla G \nabla^{\circ} L$

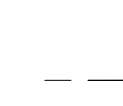

$-3.02$

$-3.12$

m.

$\stackrel{0}{+}$

2.09

-

1.00

ำ<smiles>CC(=O)/C=C(/C)[C]C(=O)c1c([AlH2])cccc1Cl</smiles> 


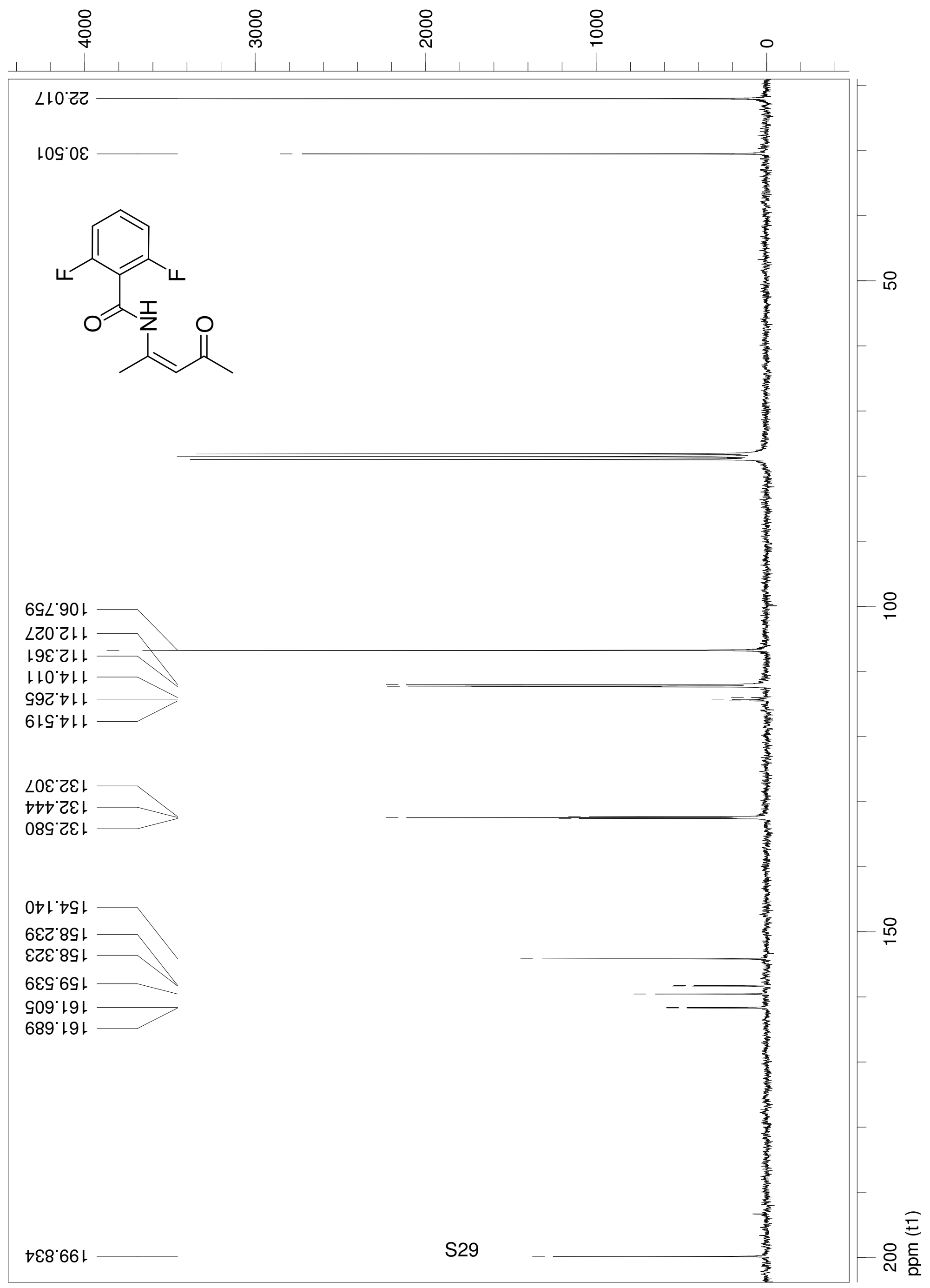


\&

웅

ट०'ट

$\varepsilon 09^{\prime} \mathrm{Z}$

$909^{\circ} \mathrm{C}$
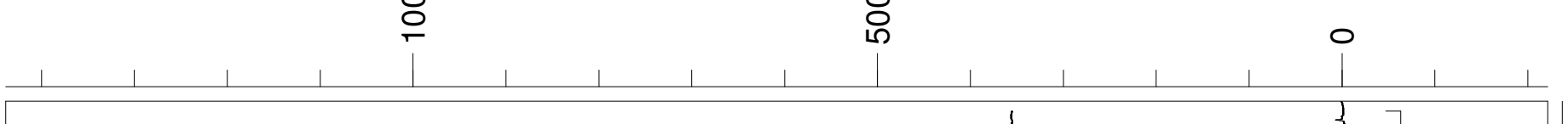

$\varepsilon \varsigma 8^{\circ} \varepsilon$

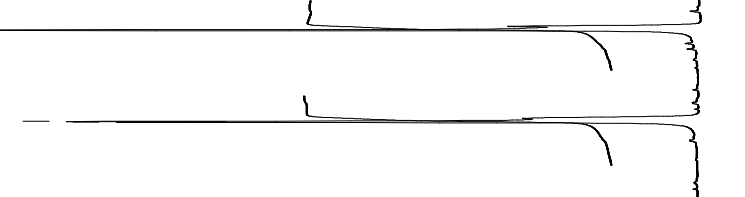

3.01

3.07

LZヤ゙G

$\angle \nabla 6^{\circ} 9$

$\angle \angle 6{ }^{\circ} 9$

$2 \angle 6^{\circ} \angle$

$200^{\circ} 8$

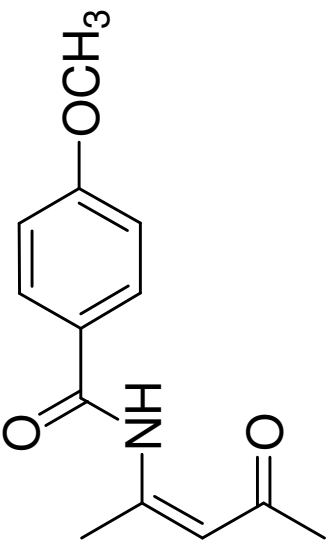

$-3.10$

$\stackrel{\circ}{\circ}$

ำ

웅

$-2.04$

은

1.99

$\infty$

i.

E 


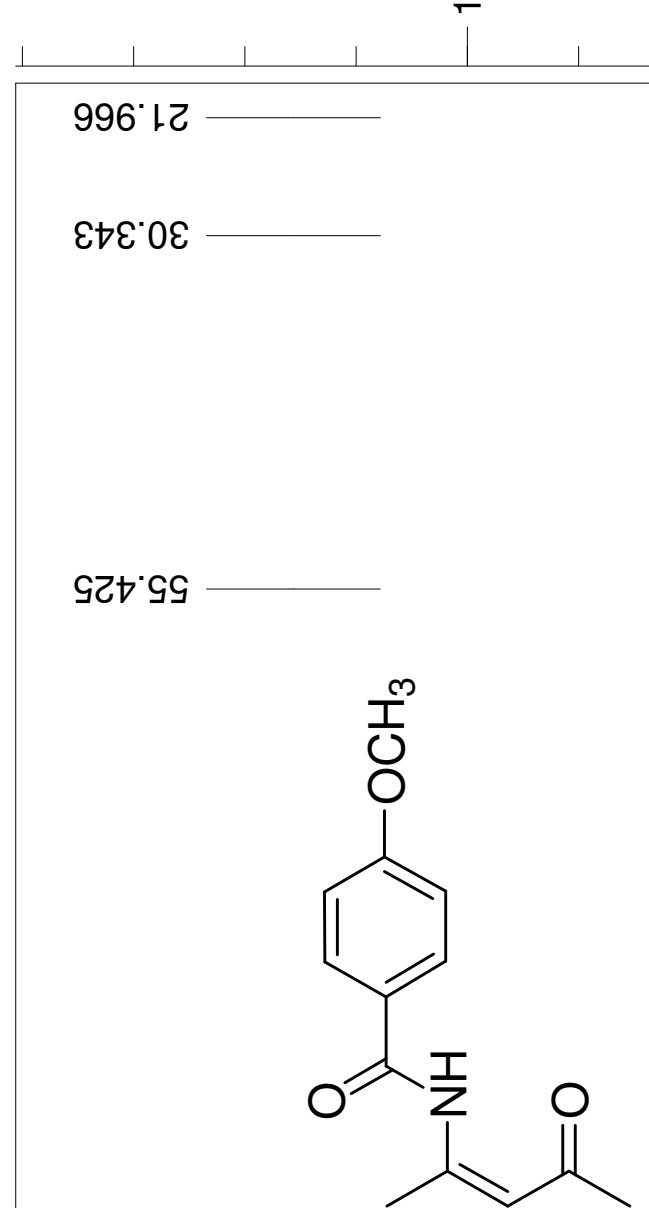

†99. 90 L

ட00ナルト

8L8 9ट

$\angle 96^{\circ} 62$ I

8เจ゙9ง

St0.89

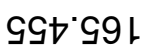


$\downarrow \varepsilon L^{\circ}$

$\varepsilon \varepsilon$ L $^{-}$

6SL'เ

$\mid \angle 6^{\circ} L$

$\angle 10^{\circ} \mathrm{C}$

IGE'Z

9Gะ'乙

$\varepsilon 9 \varepsilon " ح$

$\angle L \varepsilon^{\circ} 乙$

$16 L^{\circ} \mathrm{C}$

$9+8.2$

응 

옹
융
응
응
응

เ96.9 |

$266^{\circ} \angle 1$

$99{ }^{\prime} \triangleright 乙$

乙९9॰ ¿ह

019.87<smiles>C=C(C)/C=C(/C)[Te]1CCCC1=O</smiles>

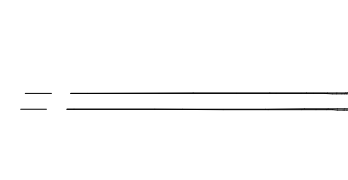

오

응

† レ8ヤレト

900 8 ト

066" $9 \varepsilon$ เ

6ヤL゙0ヤト 
g

임

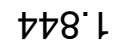

$898^{*}$ เ

$\angle 16^{\circ}$

$9 \varepsilon 8^{\circ} t$

$\angle \angle 8^{\circ} D$

0
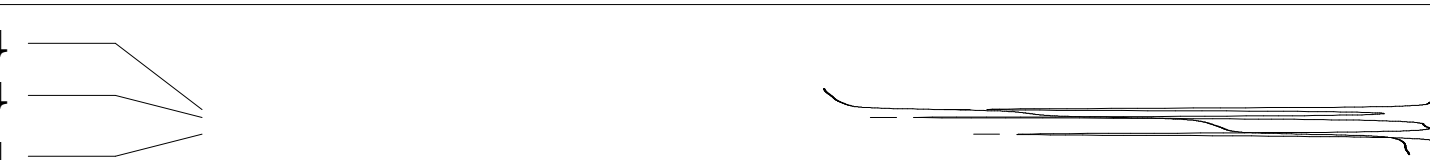

$829^{\circ} 9$

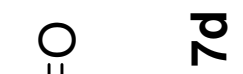<smiles>C1=CC=C1</smiles><smiles>[CH]/C(C)=C\C(=C)C</smiles> 
용

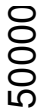

G89' เट

$\varepsilon 6 \varepsilon^{\circ} 乙 2$

$6 เ 6{ }^{\circ} \mathrm{C}$

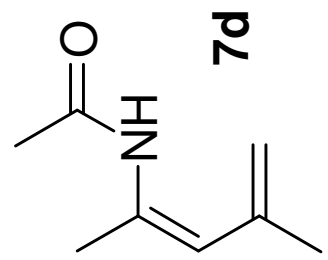

เ9゚เト

$886^{\circ}$ เ乙L

૬てヤ゙こと

ย己ट゙0†เ 


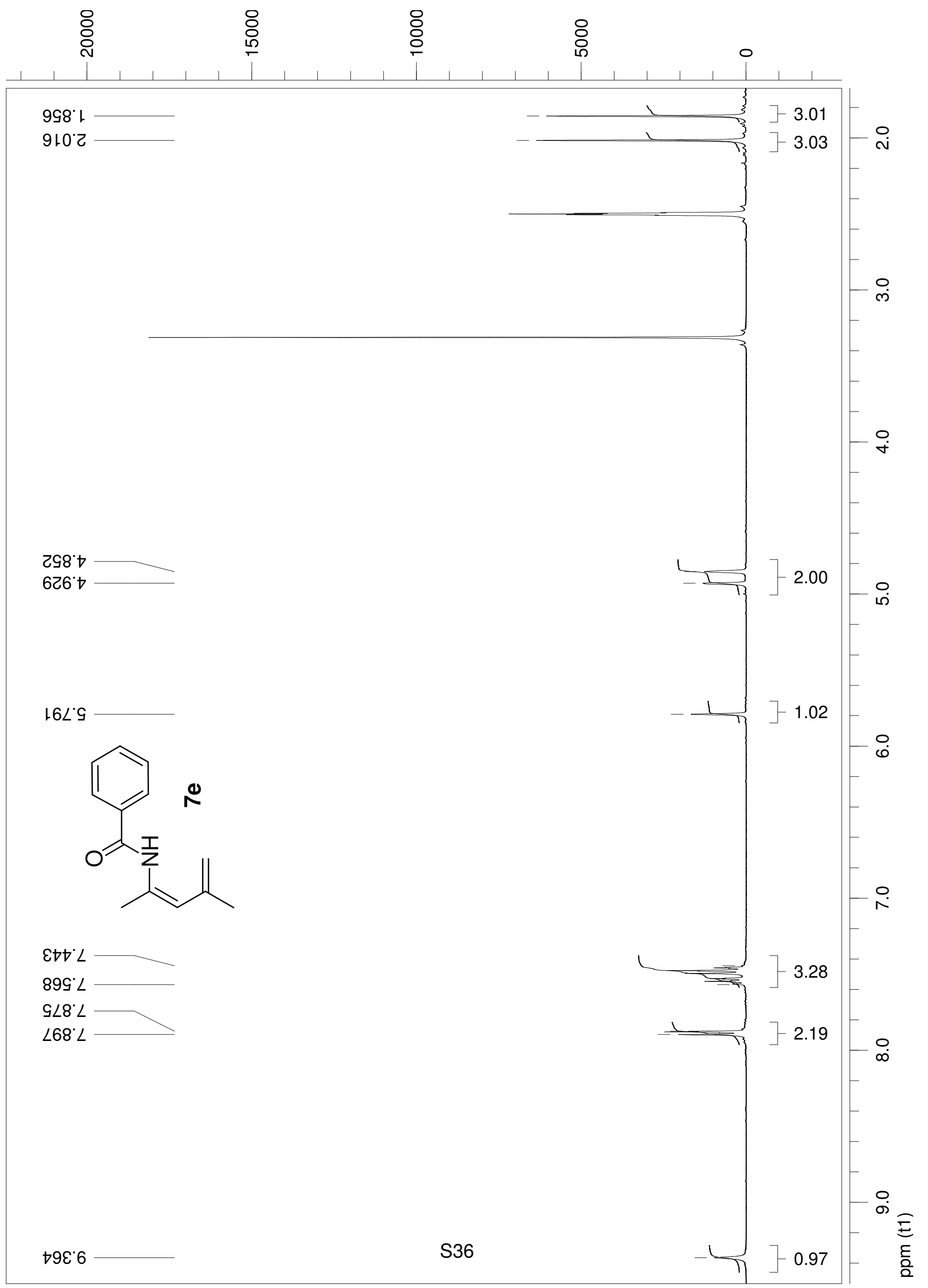


总

6LE'เ乙

५ट9॰टટ

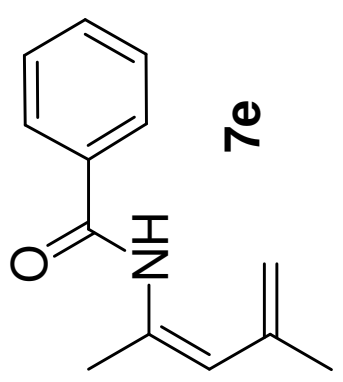

6ع0.9เ ᄂ

SL0.91

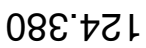

टદ己 L乙

†ยट 8टเ

घ9ट เEเ

टยเฉこเ

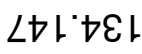

98ガ0カト

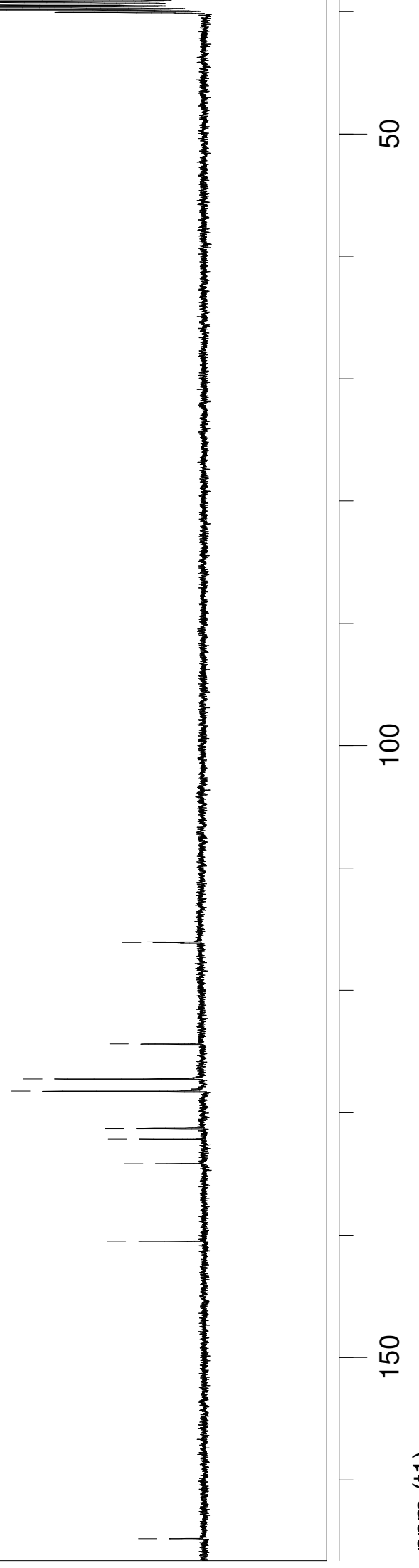

E
$\frac{\text { 을 }}{\text { 을 }}$ 
0

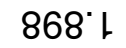

0202

96t'

$009^{\circ} \mathrm{C}$

$\checkmark 09^{\circ} \mathrm{C}$

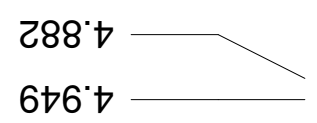

$9 \varepsilon L^{\circ} \mathrm{G}$

OSI'L

$0 \angle L^{\circ} L$

$06 L^{\circ} \mathrm{L}$

$187^{\circ} \mathrm{L}$

$999^{\circ} L$<smiles>C=C(C)/C=C(/C)[C]C(=O)c1c([AlH2])cccc1I</smiles>

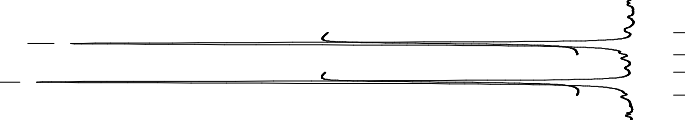

$\neg 2.97$

3.00 웅

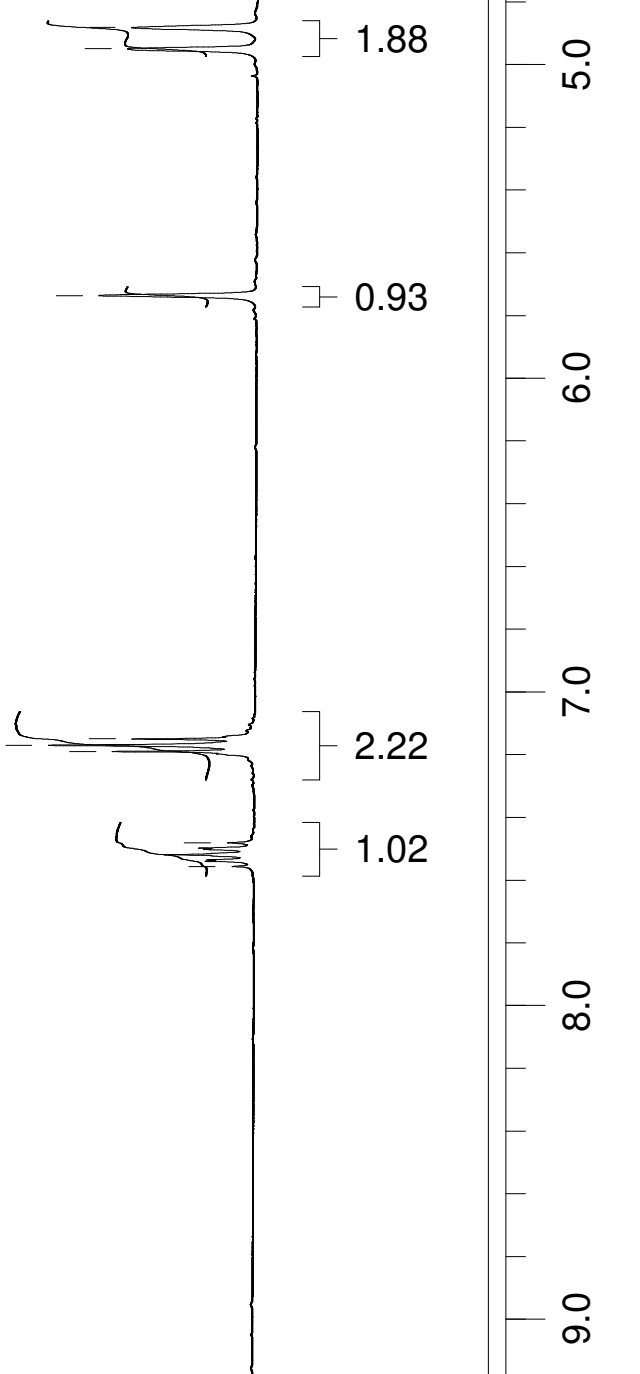




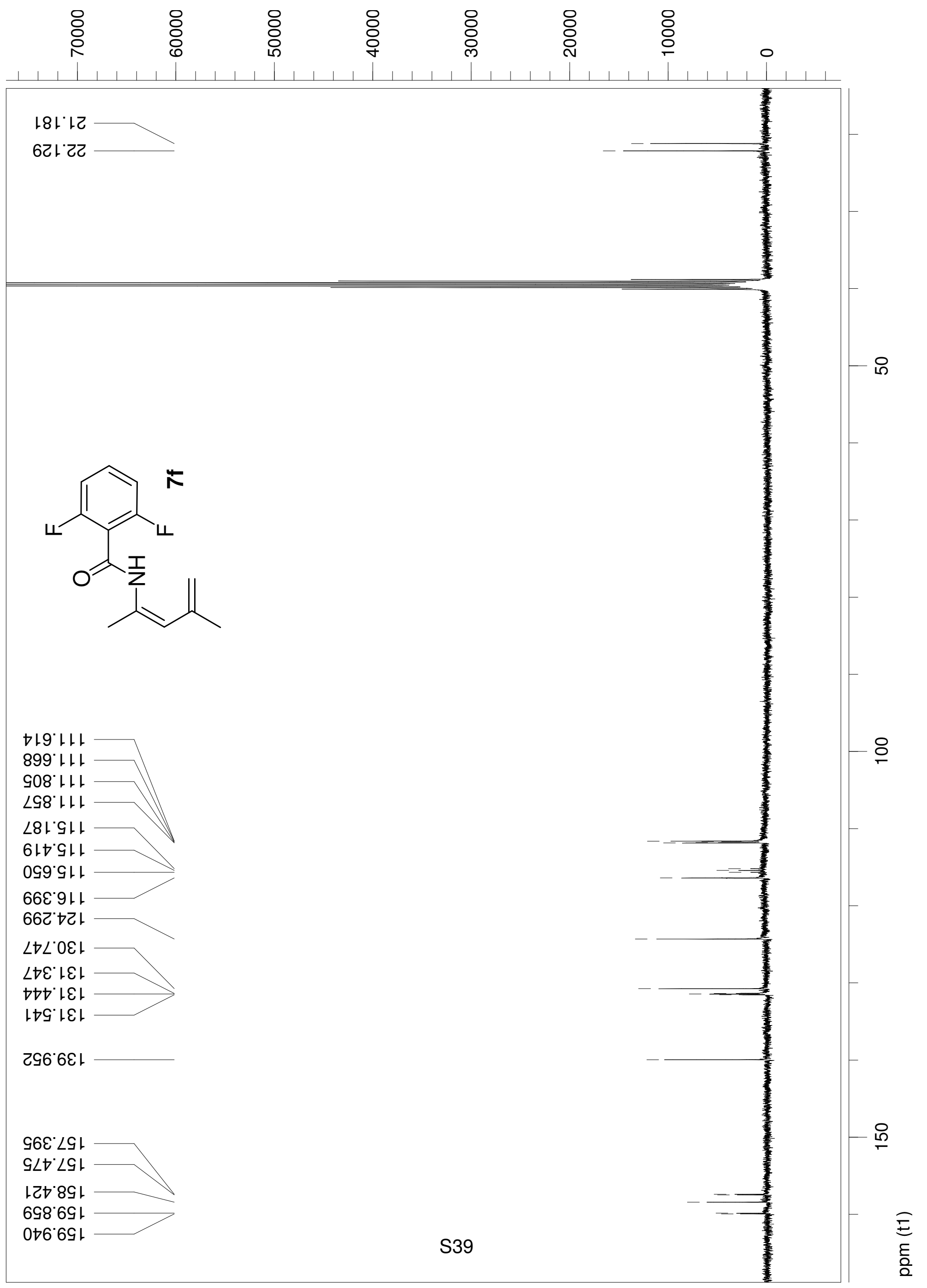


气

g

응

เ

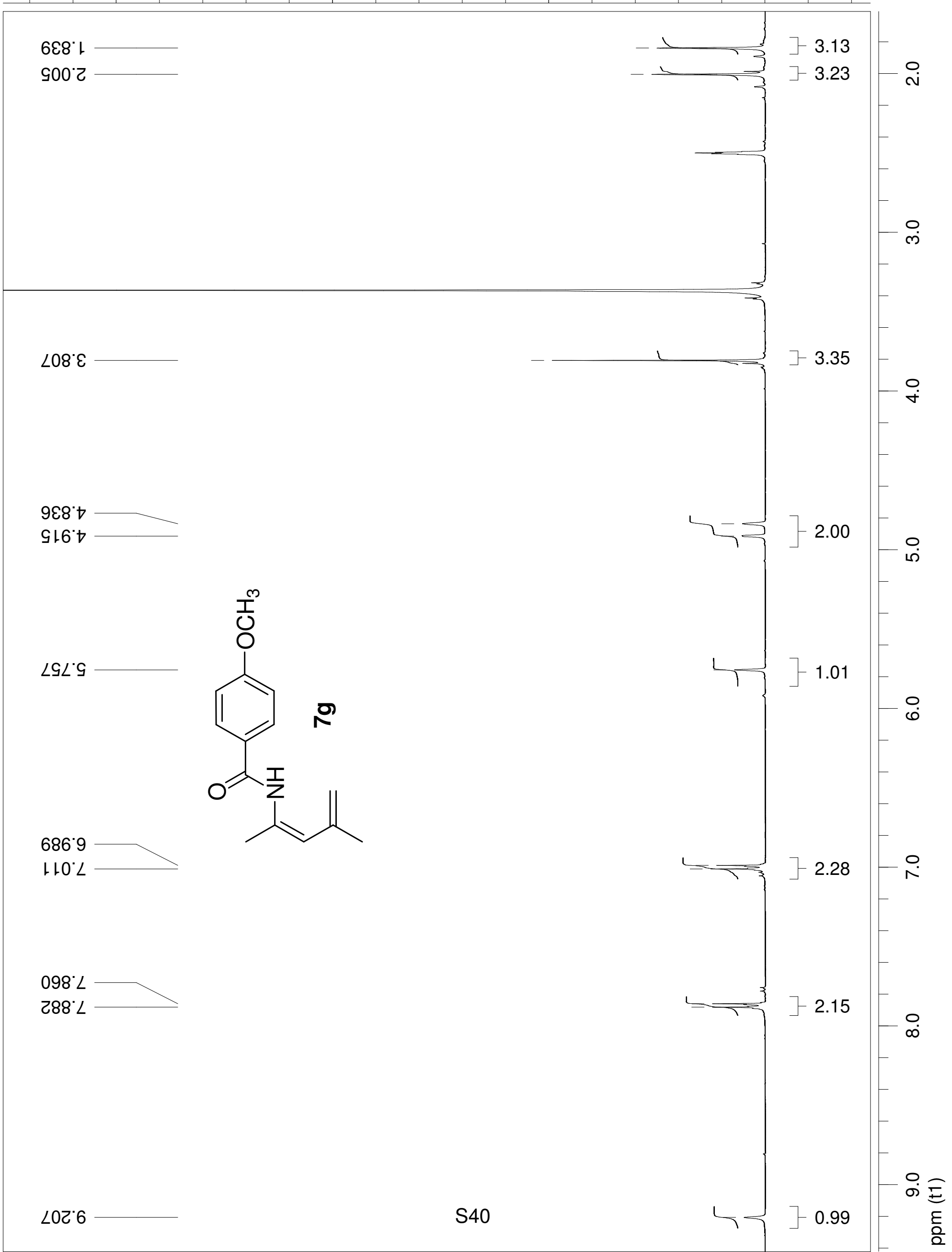


g

气

EGtเน

OSL ZZ

SIE. S

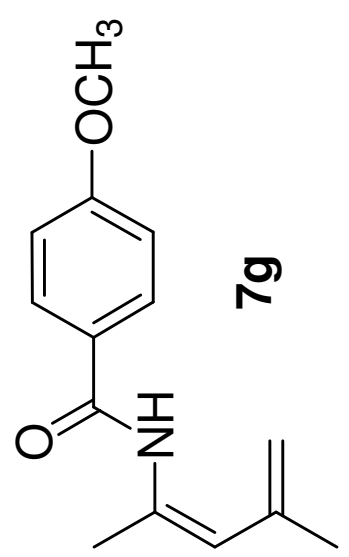

งเG๋ยเ

0L6.9ト

880॰つเ

0LE'9ट।

66เ6己

८ฤฤてこレ

†09.0ฤเ

9L9나

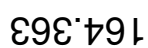

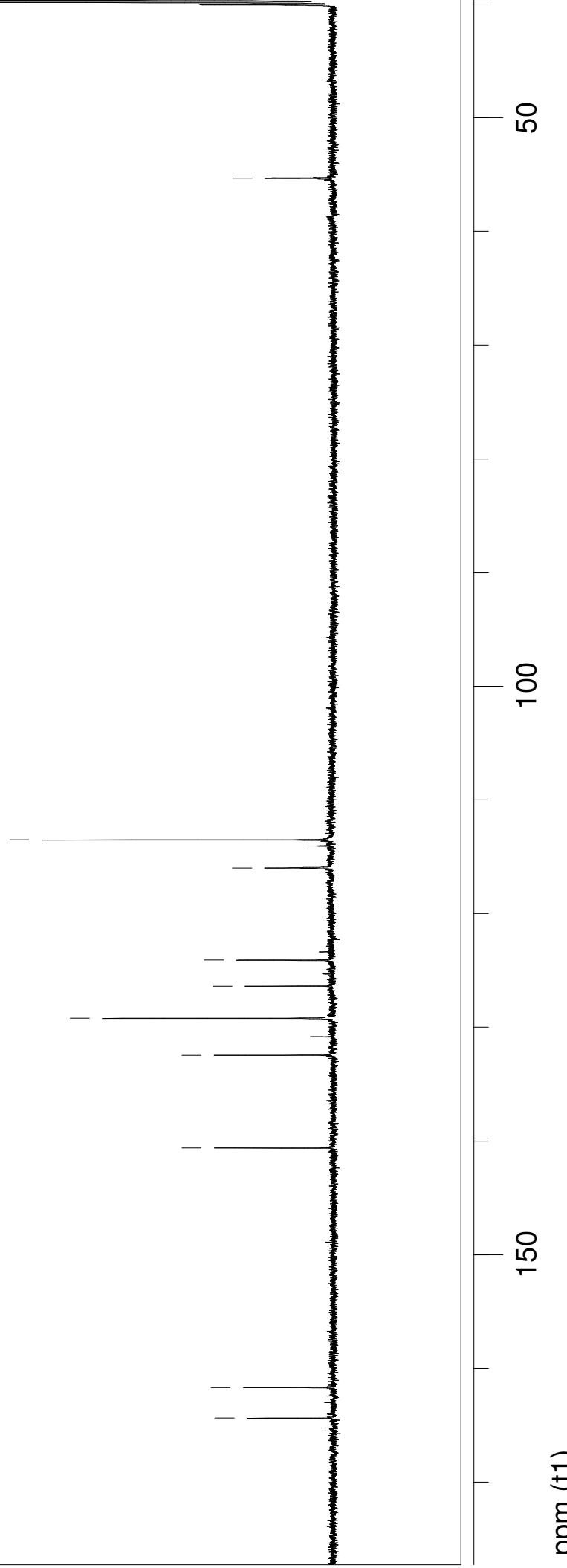

통
응 
$\S$

\&

ঃ

๙

웅

Sgl:

เ92' เ

워

กิ 으

○

ILL

6L9'Z

s99'

$\angle \angle 9^{\circ} \mathrm{Z}$

$\varepsilon G L ' Z$

$989^{\circ} \varepsilon$

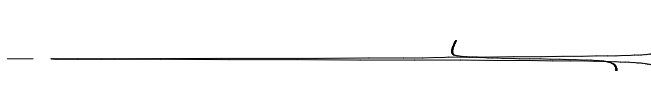

$7-2.79$

$\stackrel{\infty}{\infty}$

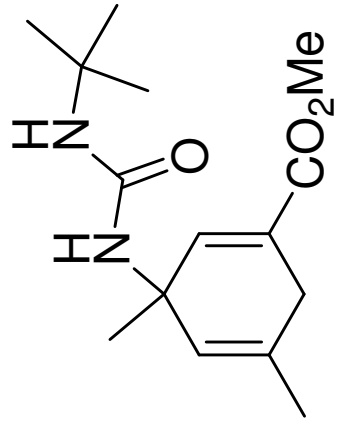

SLE' 9

s9t' 9

$96 L^{\circ} \mathrm{G}$ 


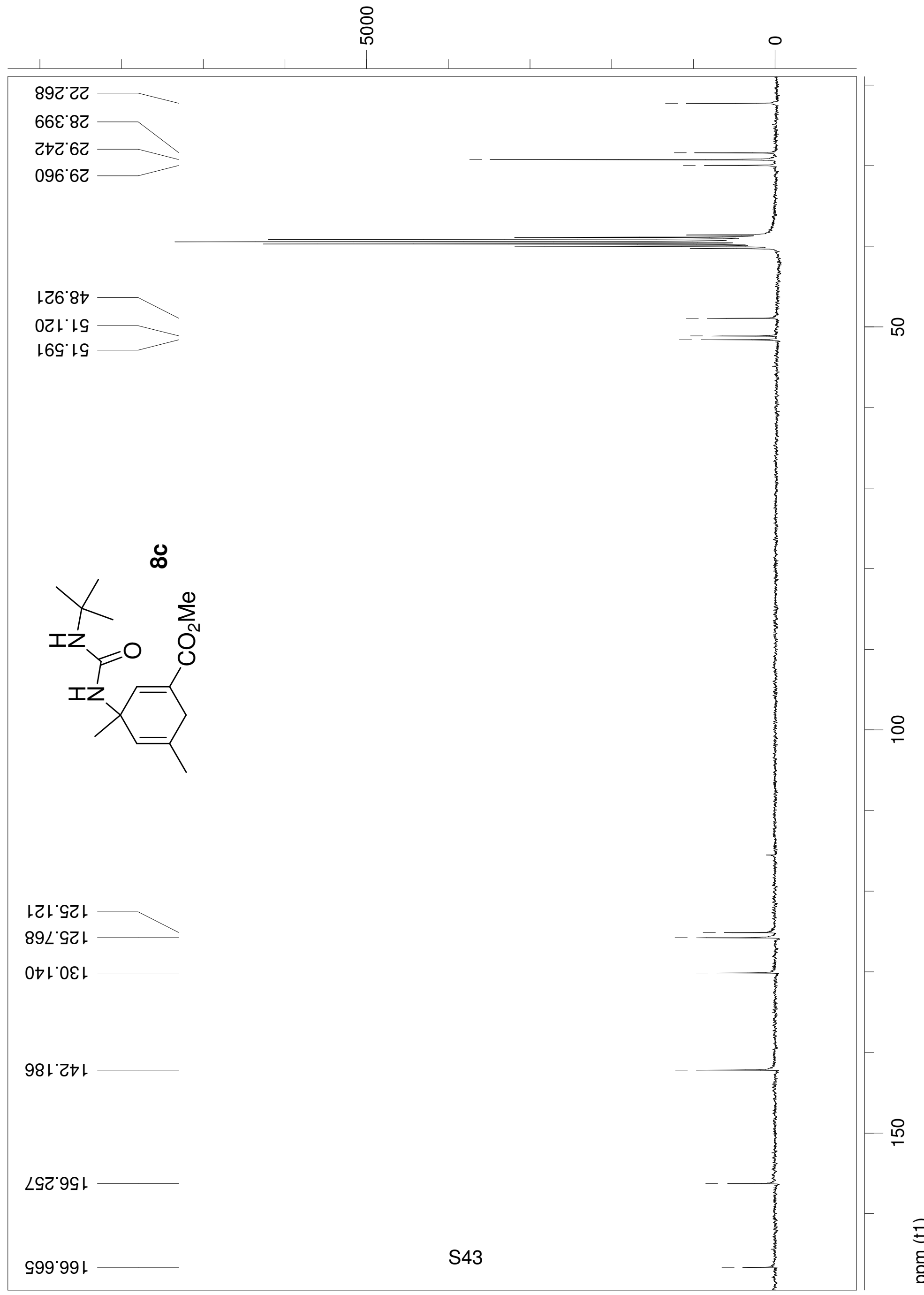


ळ

品

\&

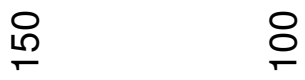

으눙

요 0

8LE'

เES เ

$\varepsilon \nabla S^{\circ} \vdash$
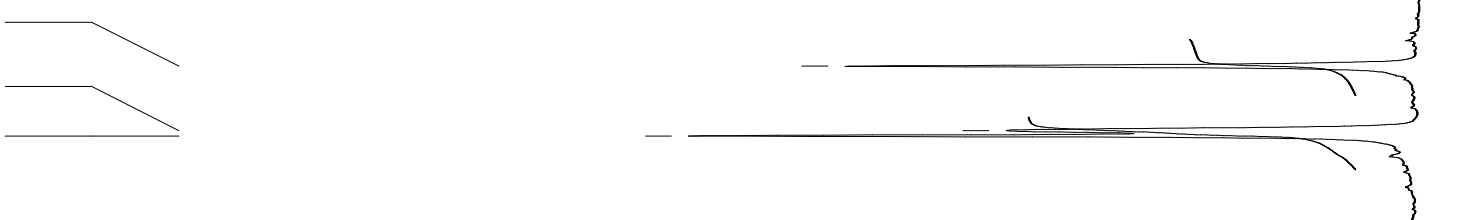

乙E9 乙

$\angle O L ' Z$

५98 2

เE6' 2

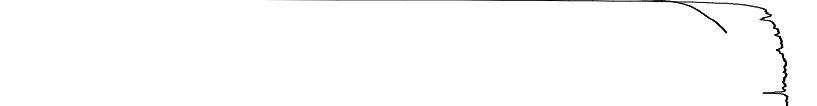

$-6.28$

8เฑ๋

ᄀ 3.00

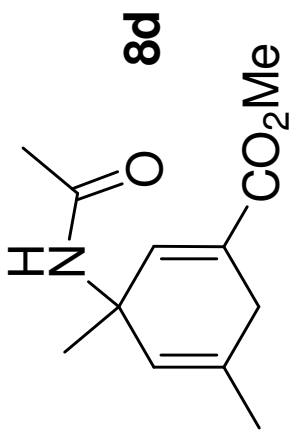

$60 \varepsilon^{\circ} \mathrm{9}$

$99 \varepsilon^{\circ} \mathrm{G}$ 


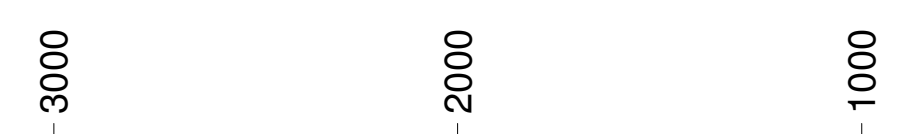

용

ఒ९९'ટટ

$\angle \triangleright 9^{\circ} \varepsilon 己$

$988^{\circ} \angle Z$

ट66.0ع

$\angle 62 \cdot 19$

L乙๐६G
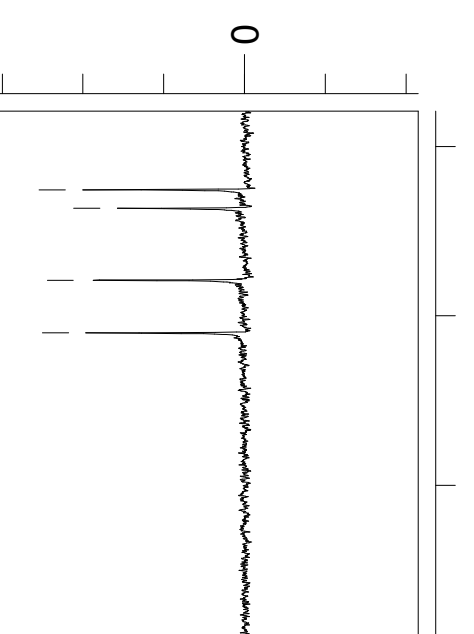

- 웅

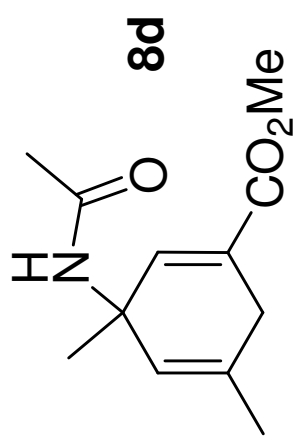

S†G`†乙

$\varepsilon \angle \nabla \cdot 乙 \varepsilon \vdash$

$0966^{\circ} 6 \varepsilon$ 


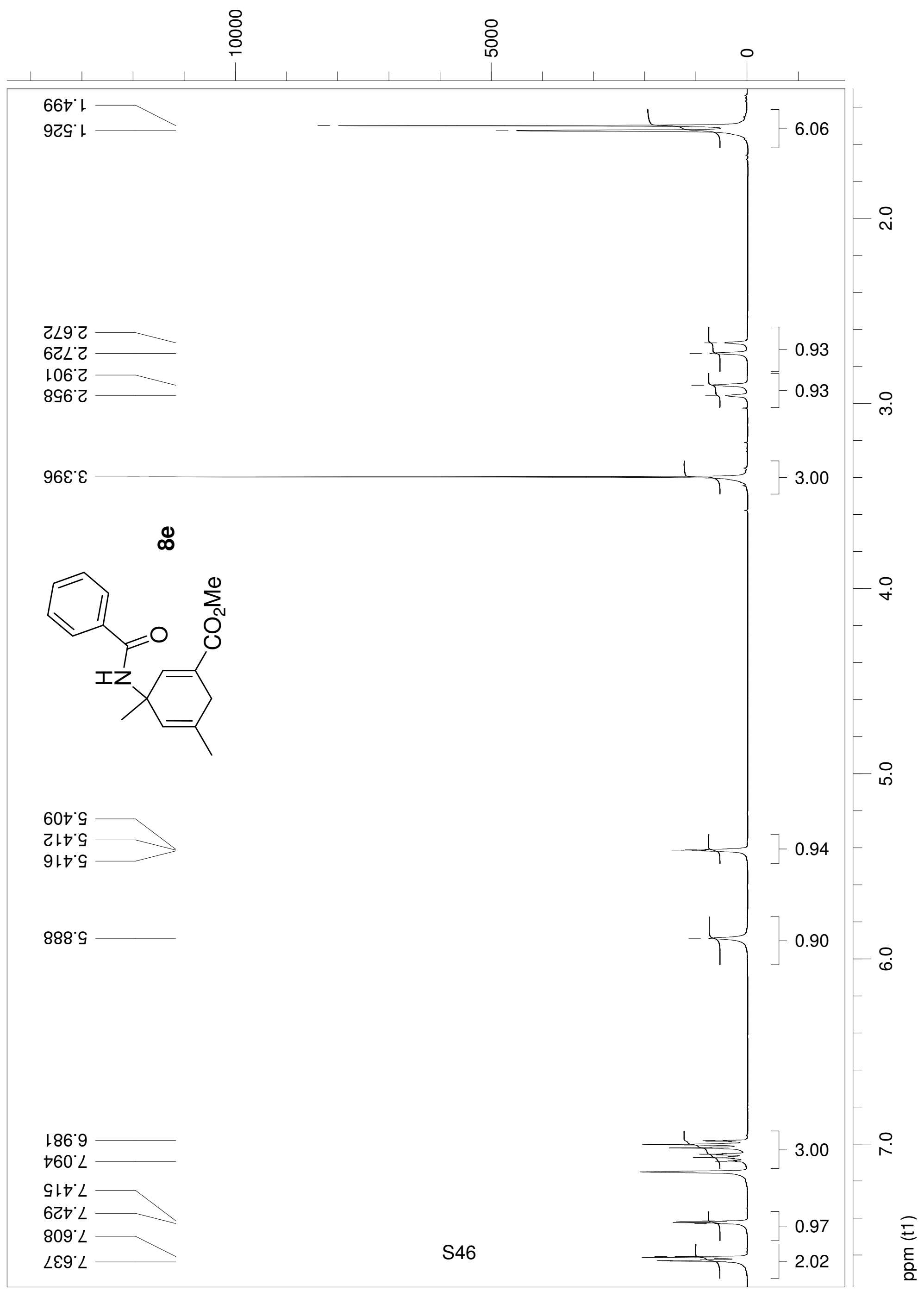


ठ

응

응

응

ঃ

응

109 Z2

$6 \varepsilon 6^{\circ} \angle Z$

$\angle 80^{\circ} \perp E$

8LC IG

૬ट9"६૬

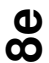

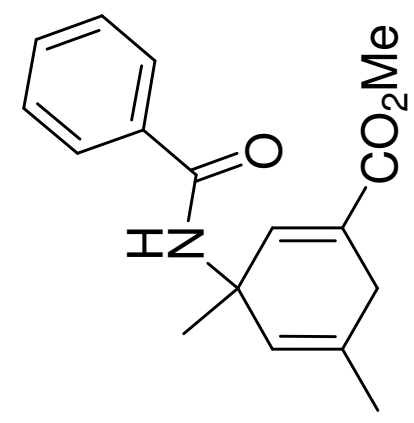

GSE๋॰ट।

ธ†ย์ L乙L

89t 821

080 เEเ

666 टहเ

L06' $9 \varepsilon$ เ

$\angle 99^{\circ} 6 \varepsilon$ เ 


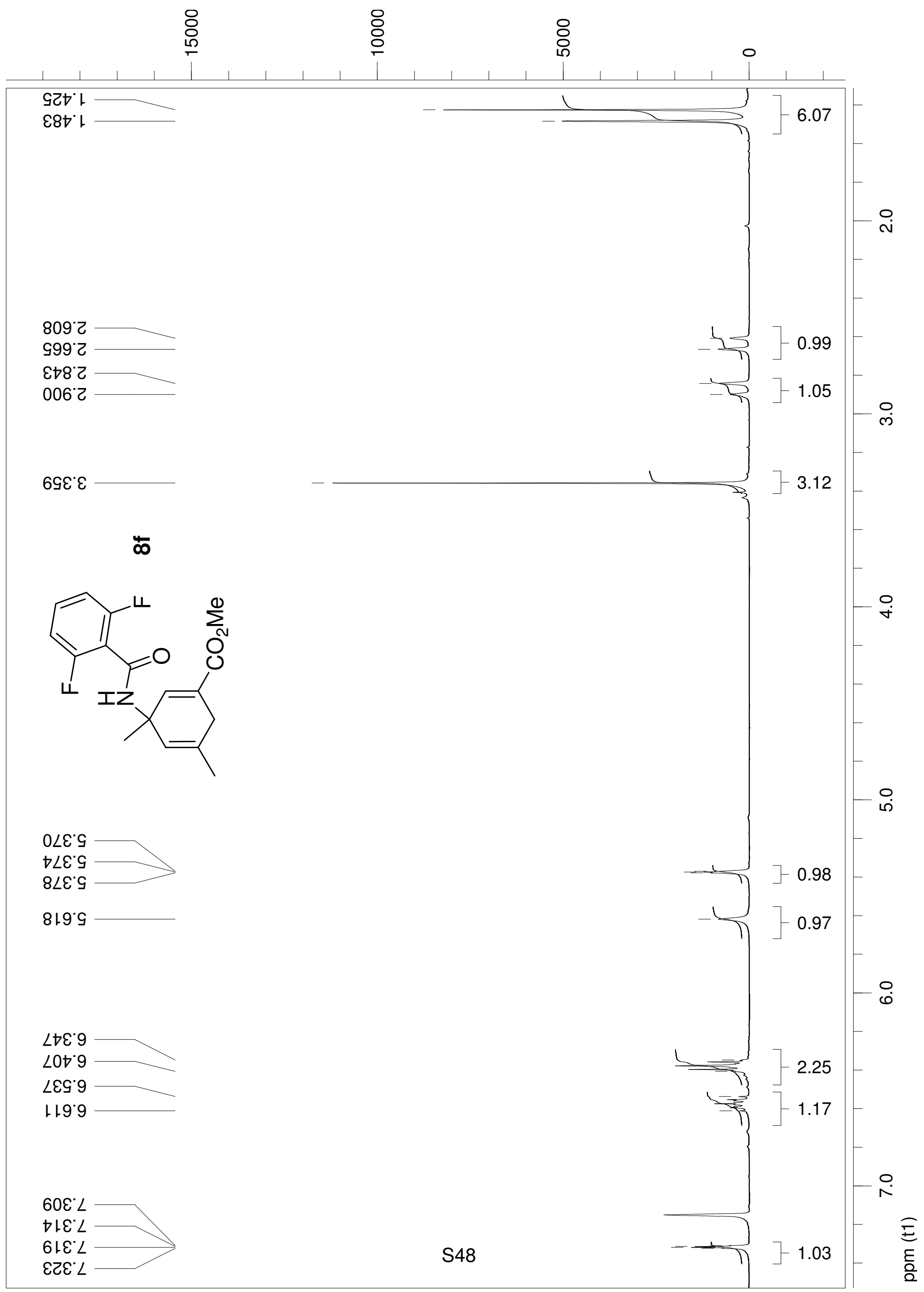




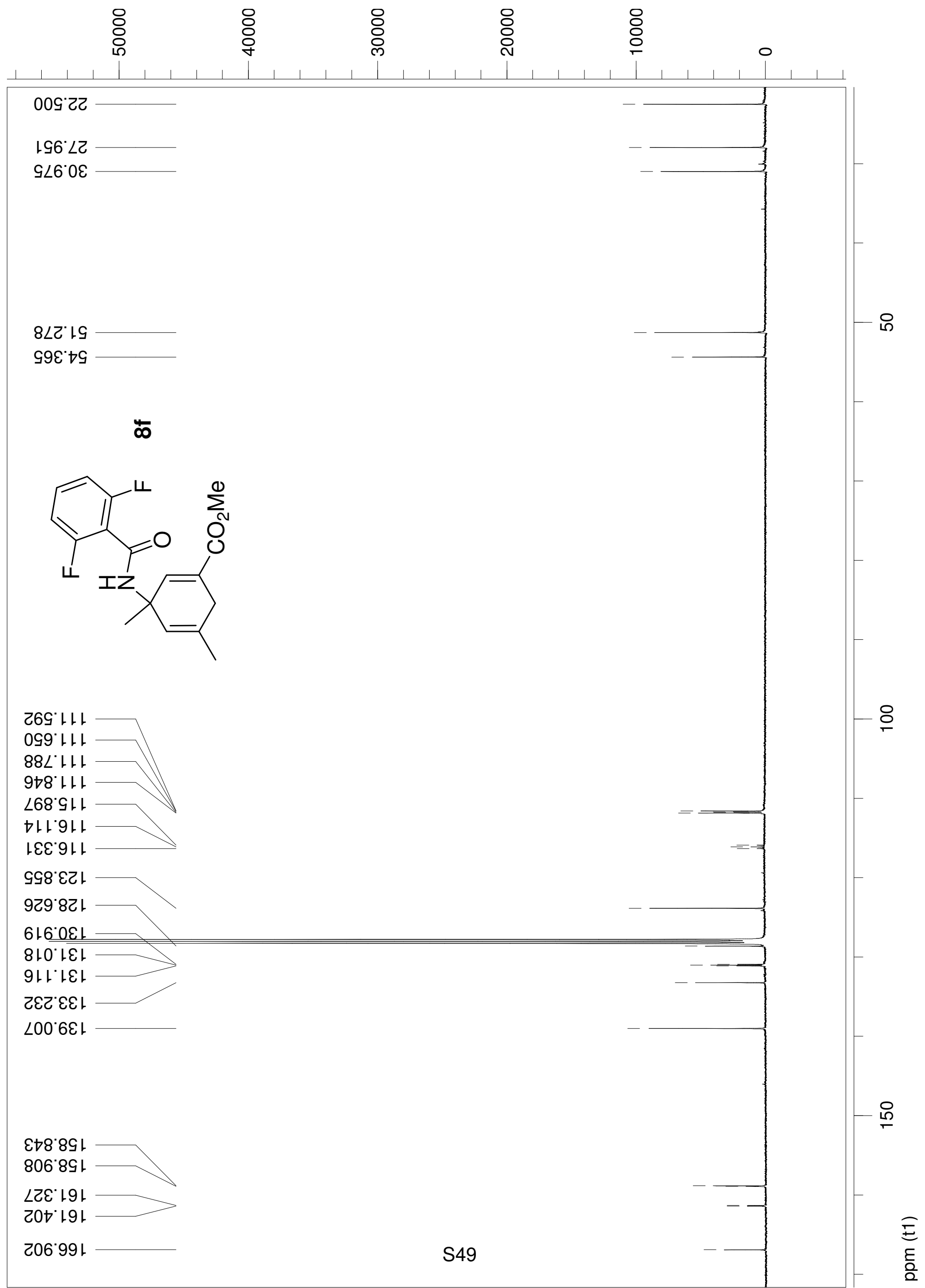


을

] 6.23

669 ح

9GL'Z

9ع6 2

$\varepsilon 66^{\circ} \mathrm{Z}$

ट८।

$\neg 6 \varepsilon^{\circ} \varepsilon$

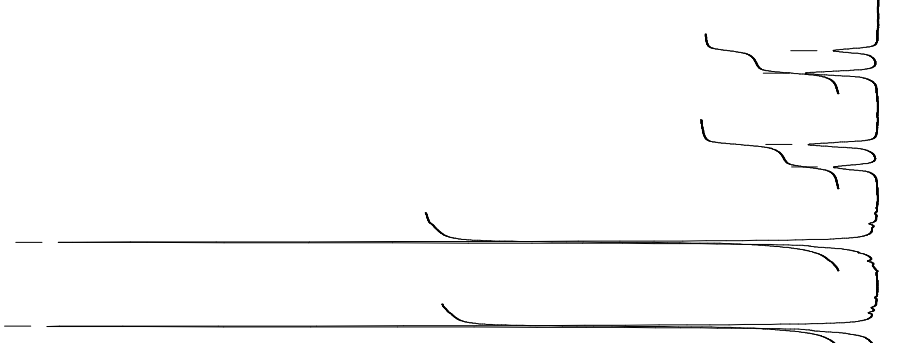

$-1.06$

$-1.09$

ले

$-3.30$

$-3.17$

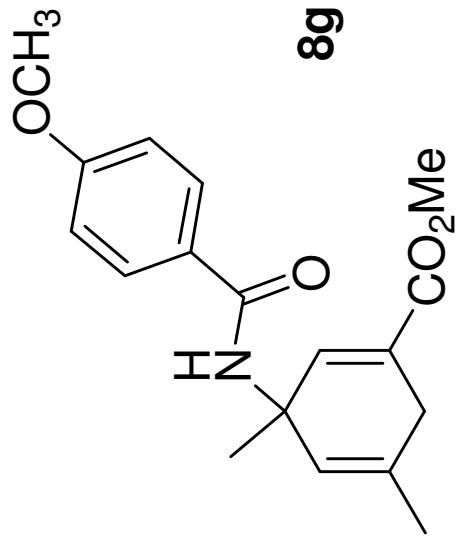

8Zt'G

†29 9

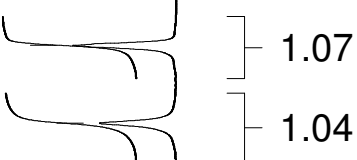

969' 9

819.9 


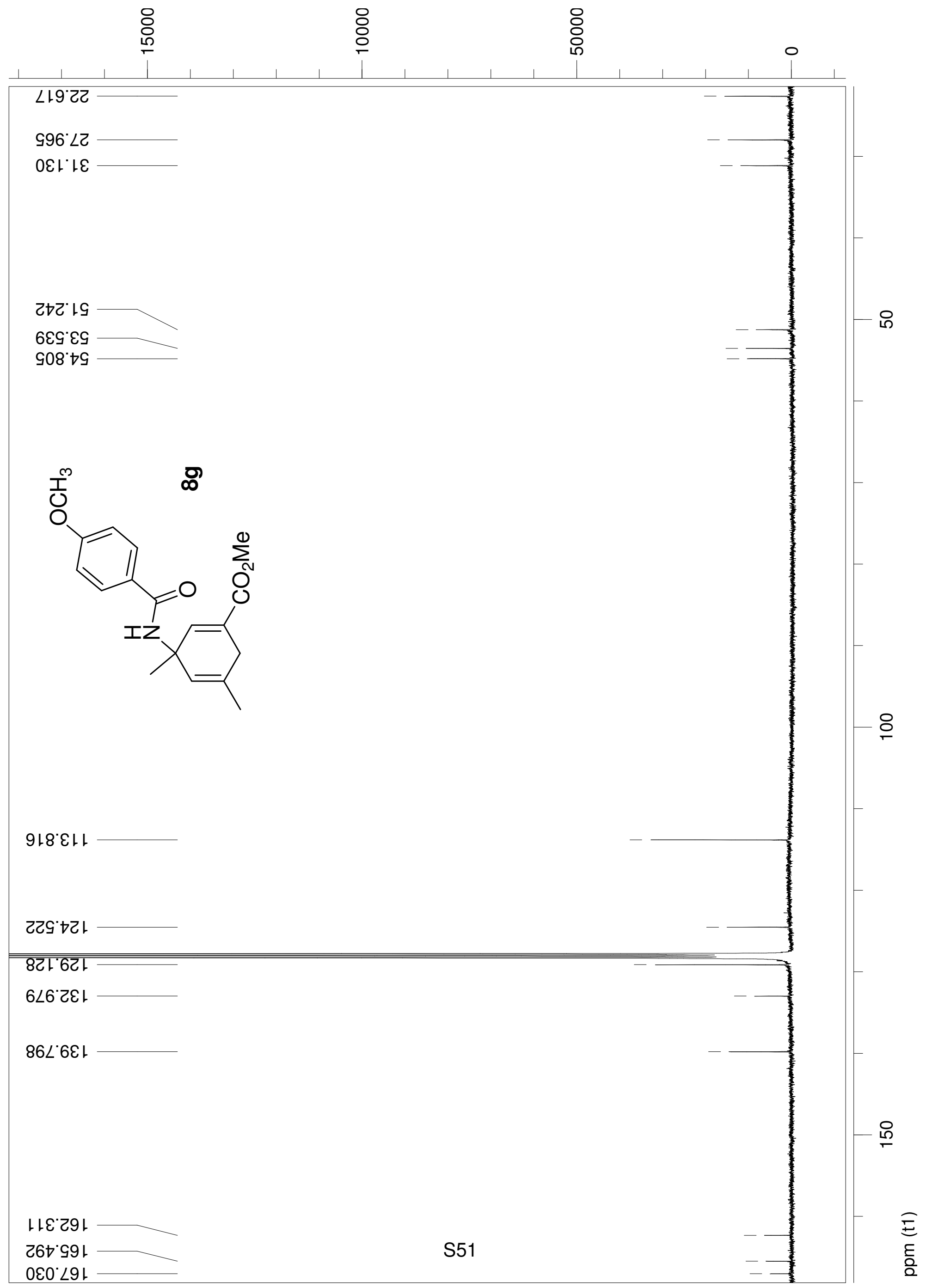


욤

음

욤

8เト๋

8LC!

$\nabla \angle \nabla^{\circ}$

$\angle \angle \nabla^{\circ}$

$\nabla G L{ }^{\circ}$

G26. -

$986^{\circ}$ เ

$009^{\circ} 2$

$0 \varepsilon 8 ' ح$

$66 \varepsilon^{\circ} \varepsilon$

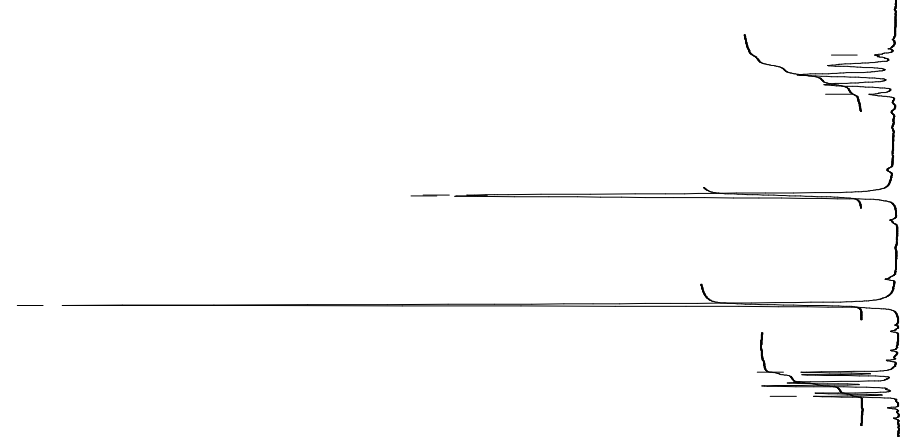

$-2.25$

$\neg-3.04$

$-3.09$

1.91

ำ

穴

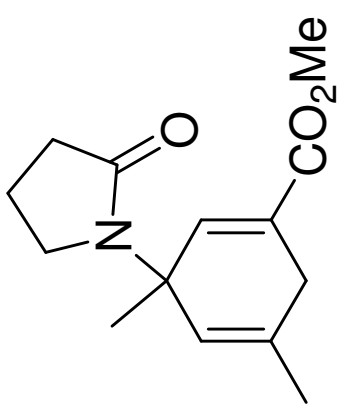

$\angle O T^{\circ} \mathrm{S}$

टाt 9

$\angle 1 D^{\circ} G$

टटt 9

8टt 9

EEt 9

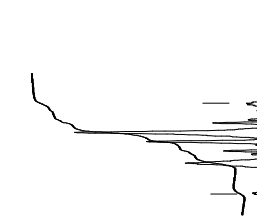




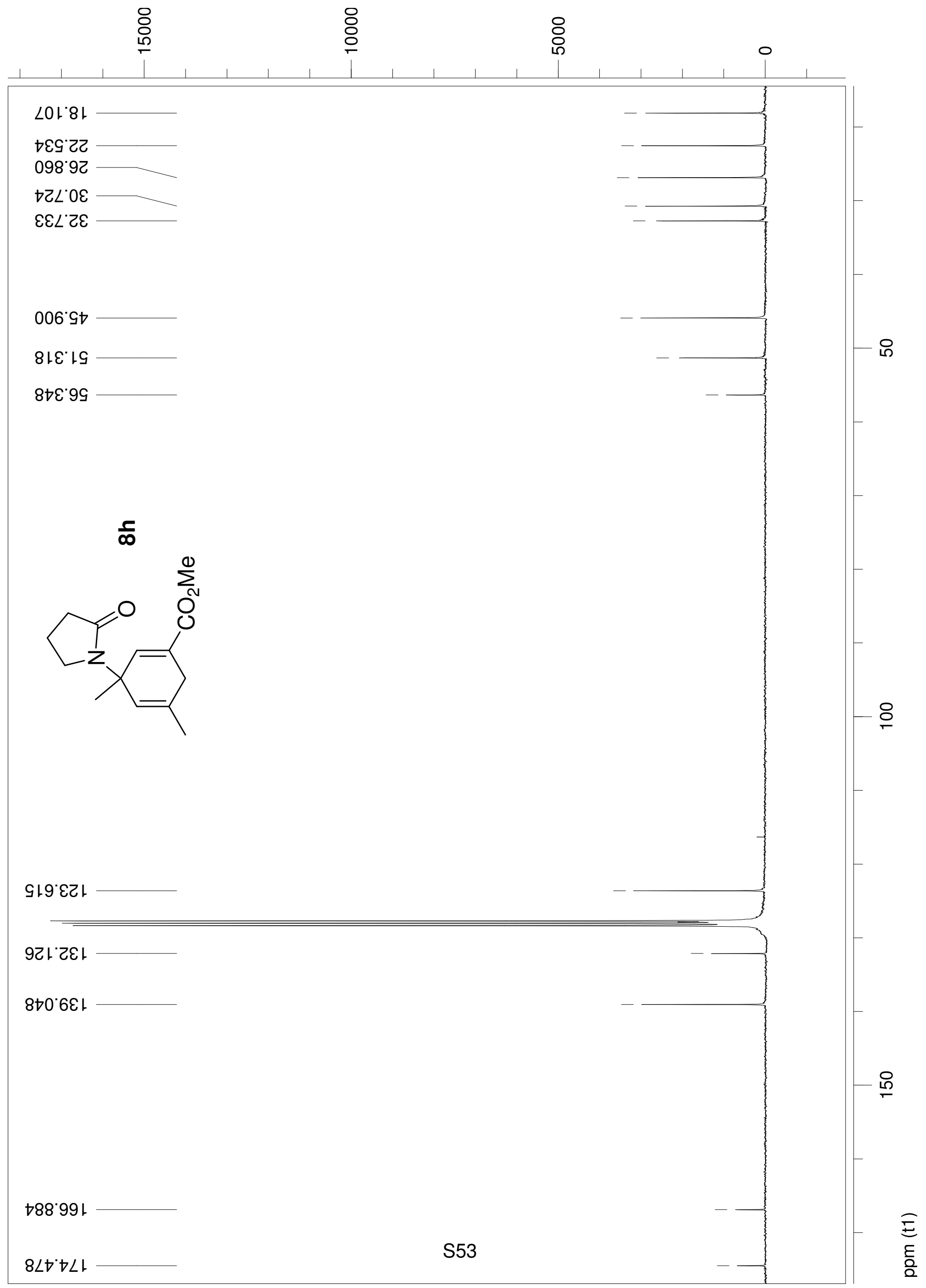




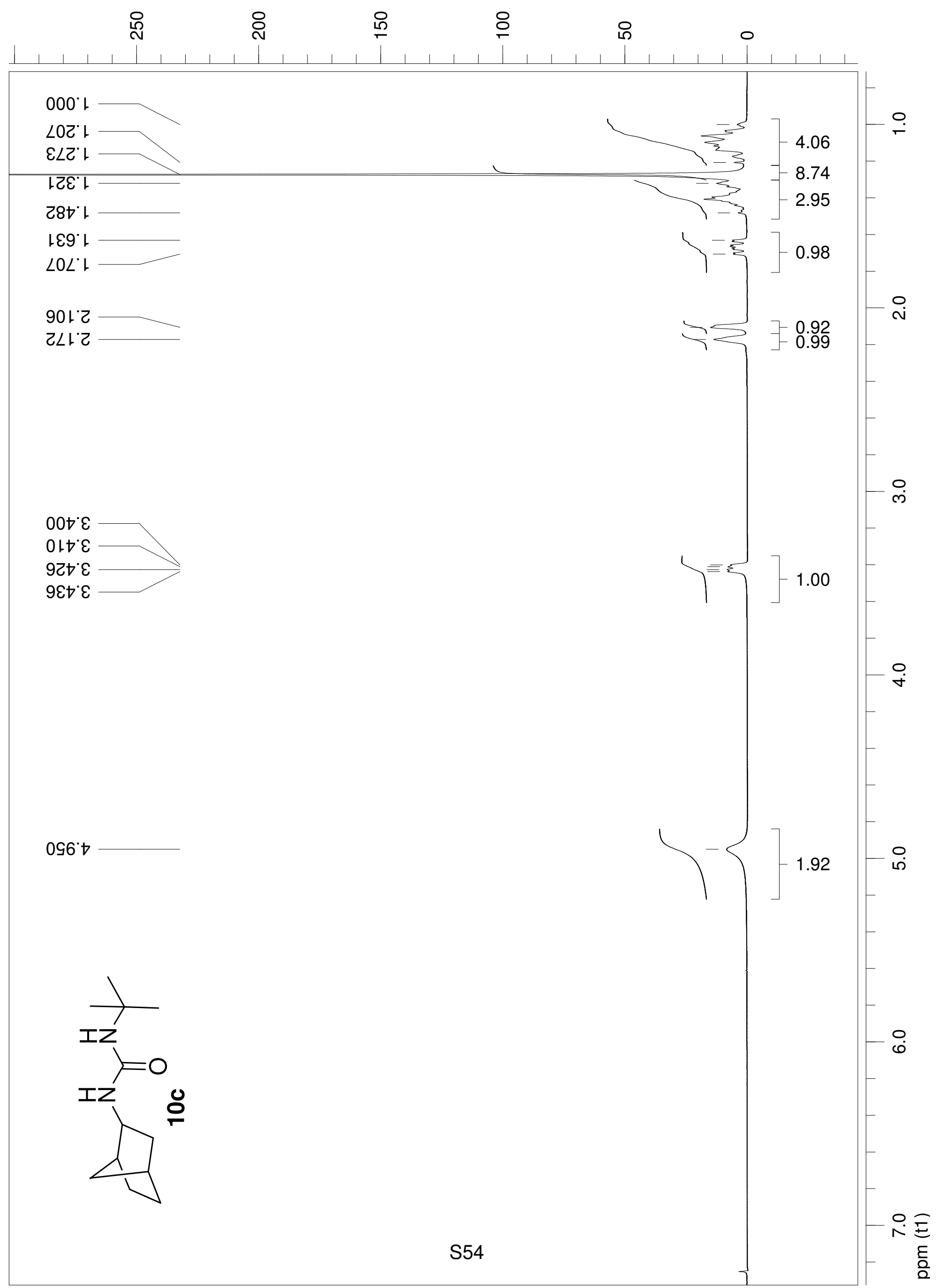


흠

亏

0ะヤ・9乙

$0 \angle 182$

6\&9'6己

60ट $9 \varepsilon$

S† 'ง

199.0t

৪टL¿๐

E96.6t

५O乙 \&૬

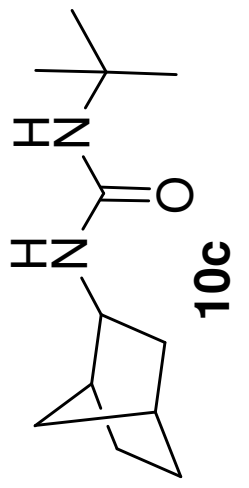




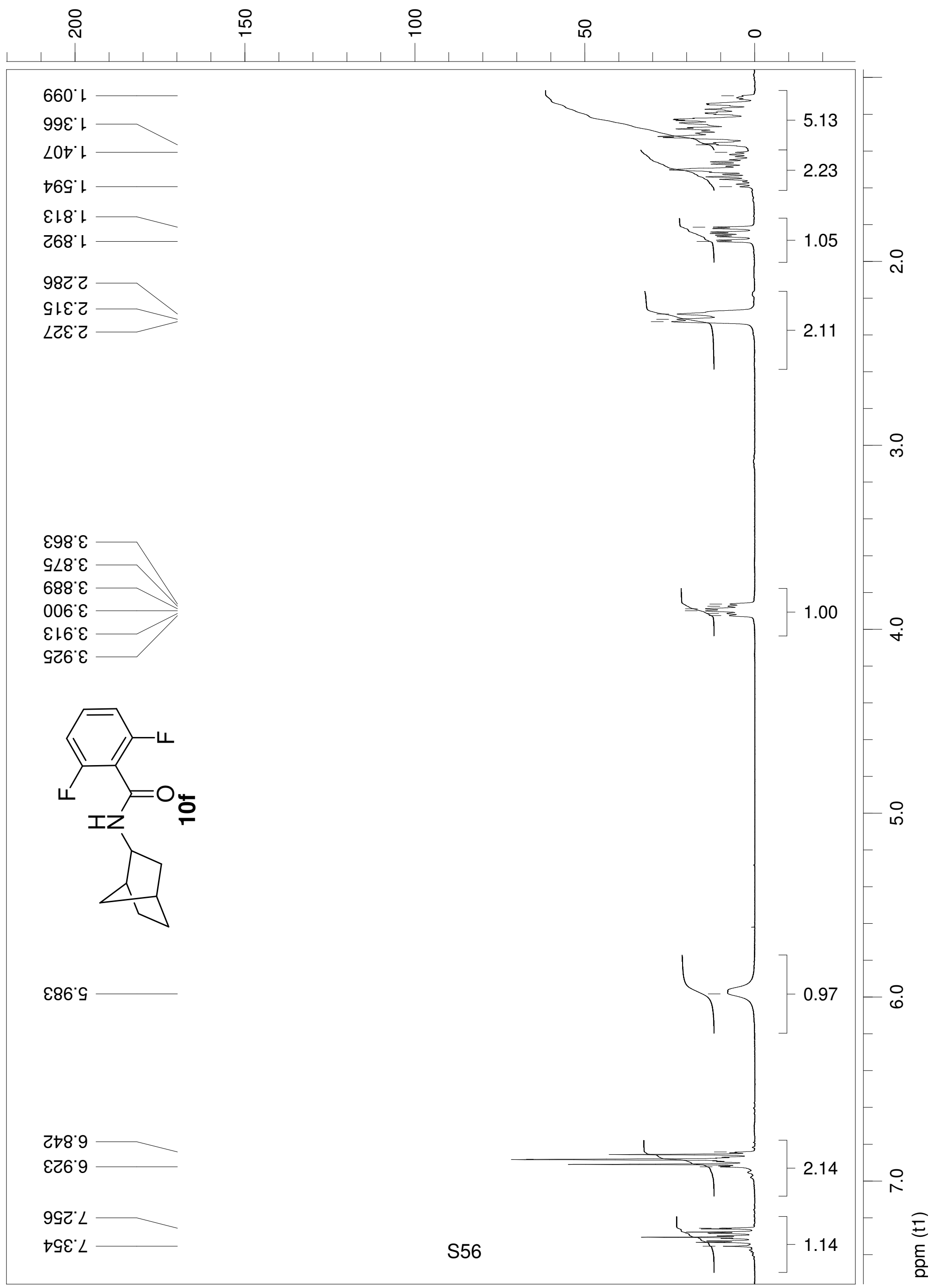




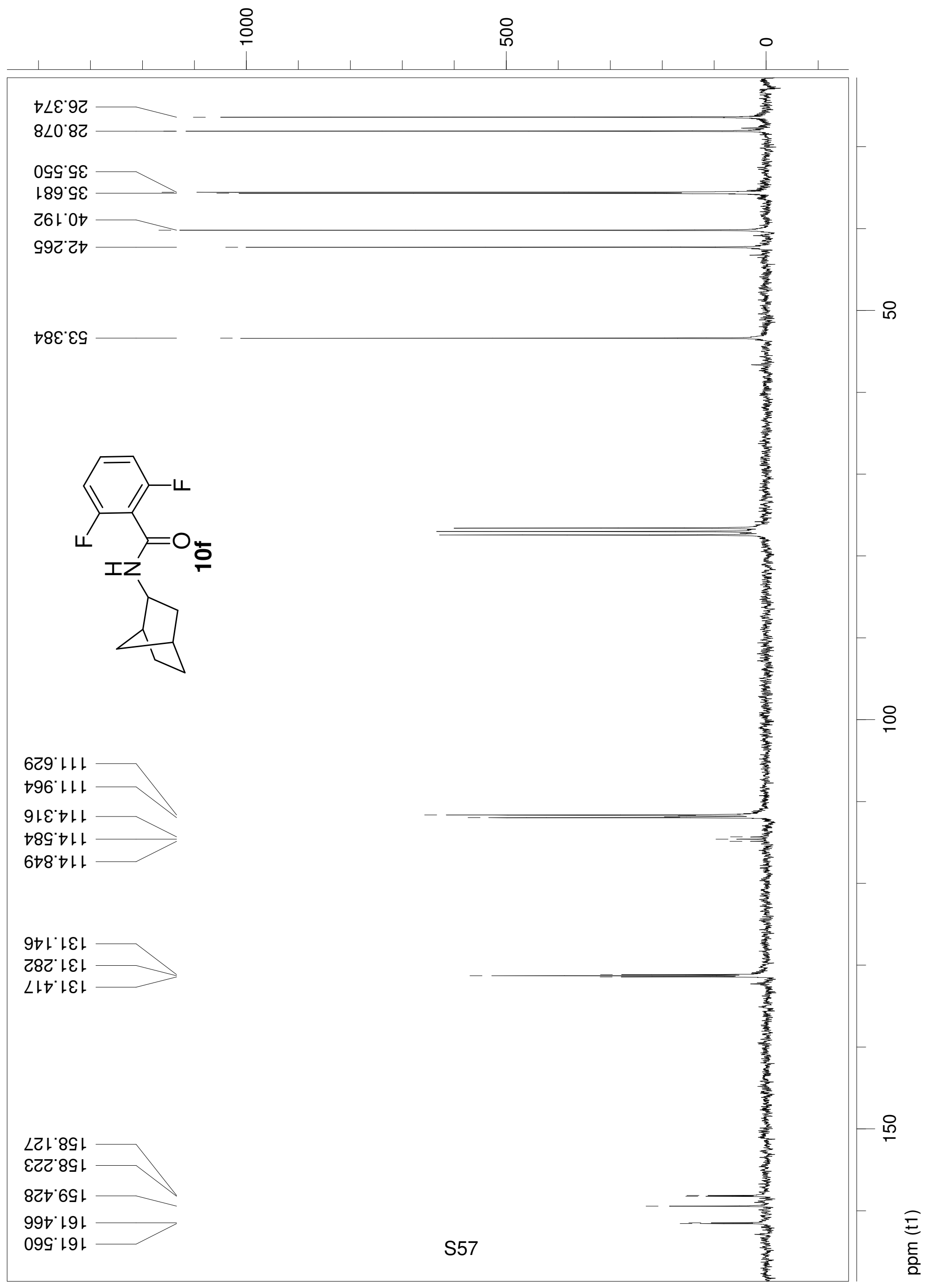




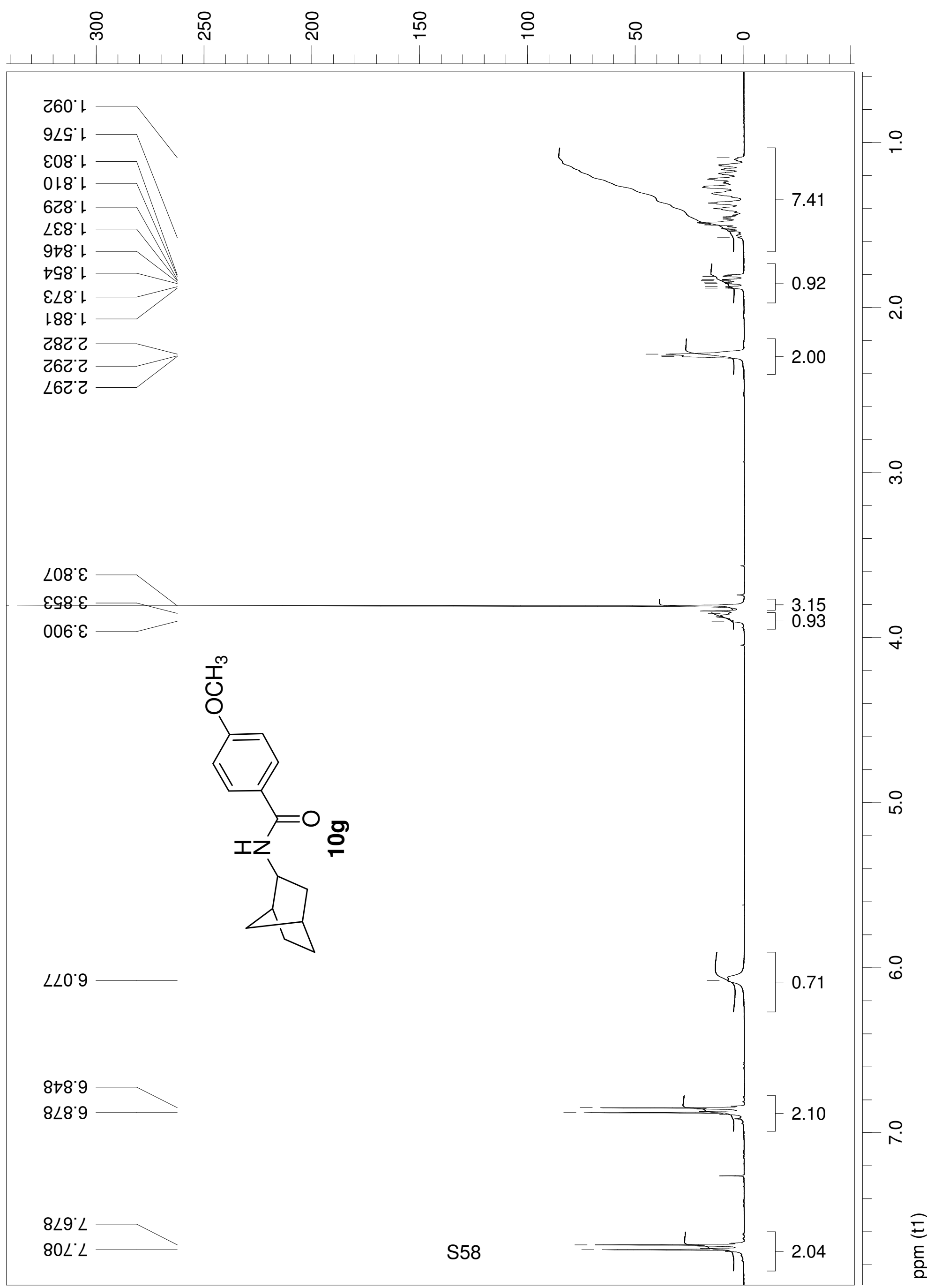




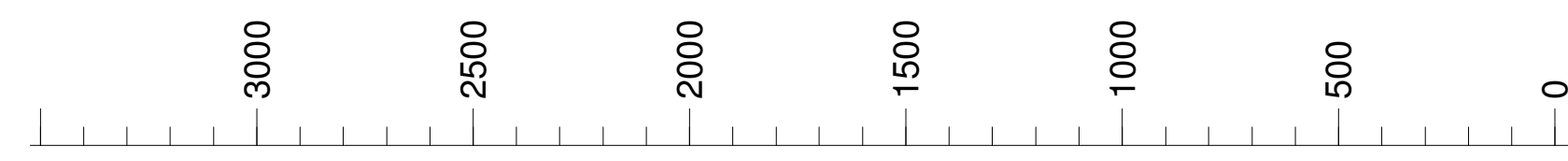

097 92

$\checkmark 60{ }^{\circ} 8 \mathrm{Z}$

เ6G' $9 \varepsilon$

6L9 $9 \varepsilon$

$\varepsilon 8 \varepsilon^{\circ} 0 t$

$\neg 9 \varepsilon$ ट

เE乙 \&૬

ह6ट 99
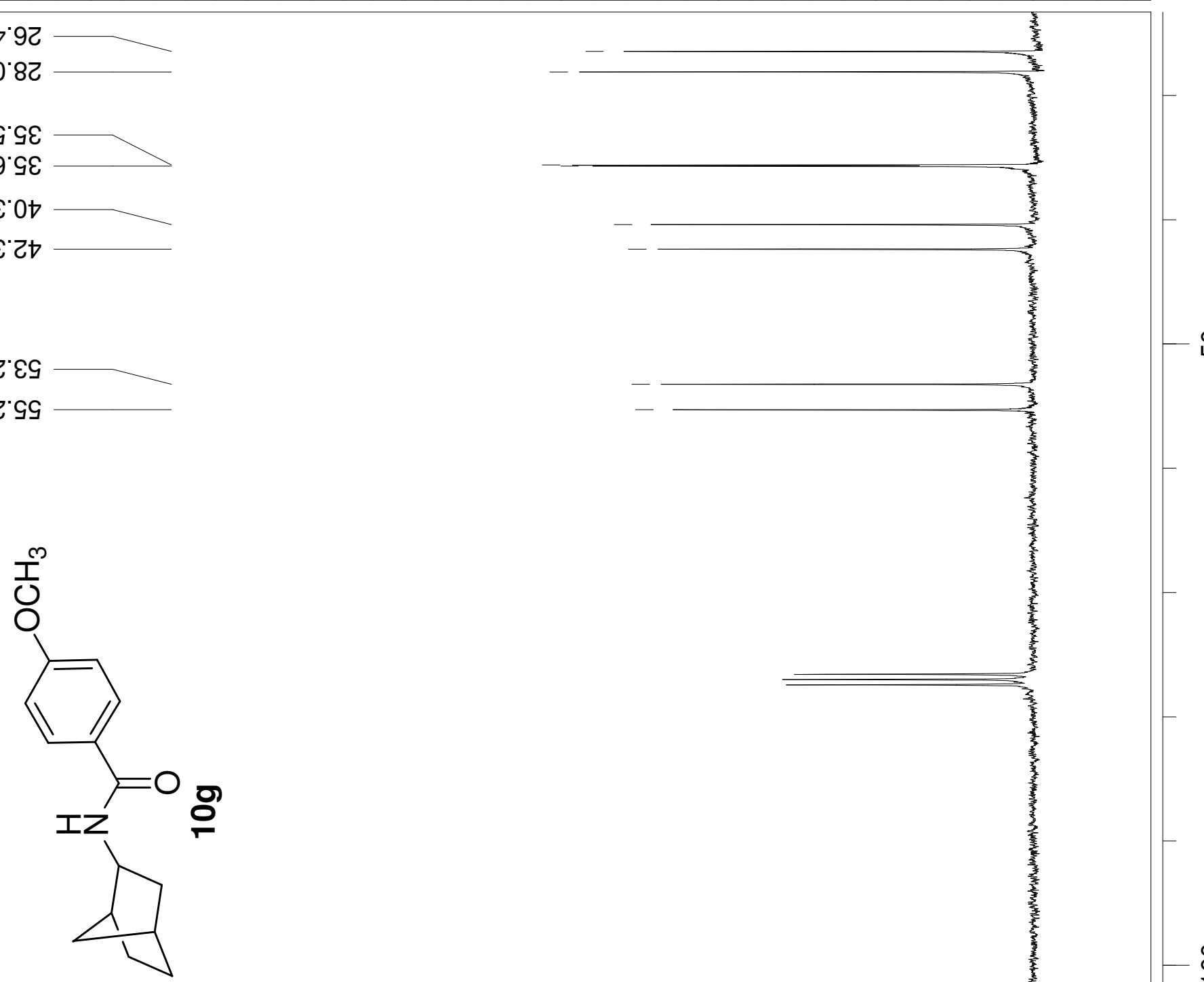

8ح9'ยเ

$\angle 90^{\circ} \angle Z L$

999.8Z

$\varepsilon 86^{\circ} เ \varepsilon$ 
ঃ

เटเ๋

9เट เ

662 เ

OtS'

ट $\angle 9^{\circ}$ เ

LEL'

8L8'

$\downarrow \varepsilon 0^{\circ} \mathrm{C}$

$\angle 91^{\circ} \mathrm{C}$

५९ट 乙

$\angle 6 Z^{\circ} 乙$

टटฑ こ

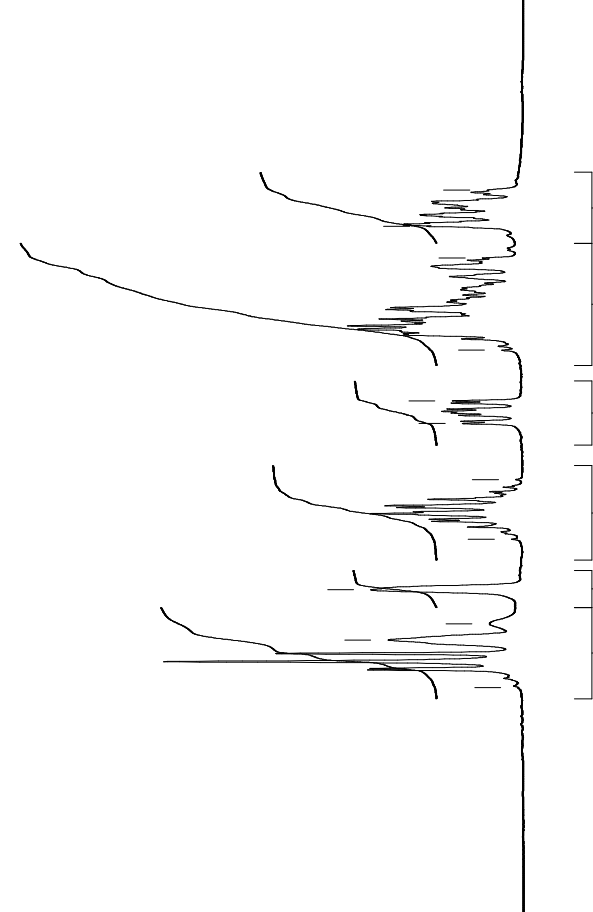

$9 เ \varepsilon^{\circ} \varepsilon$

เE๋ $\varepsilon$

$\angle 10^{\circ} \mathrm{D}$

$0 \varepsilon 0^{\circ} t$

$8 \varepsilon 0^{\circ} \mathrm{t}$

$190^{\circ} \circ$

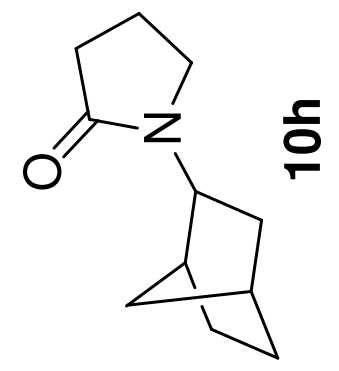


$190 \%$ L

$699^{\circ} \angle 2$

$\angle G D^{\circ} 82$

乙8.

$090^{\circ} 9 \varepsilon$

เ $8 L^{\prime} 9 \varepsilon$

$\varepsilon \hookrightarrow 6^{\circ} 9 \varepsilon$

$69 \varepsilon^{\circ} 0 t$

$6 \angle L \triangleright t$

$\varepsilon 0 \varepsilon^{\circ} \downarrow \varsigma$

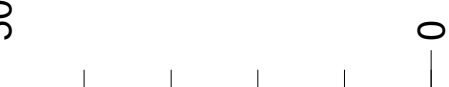


亏ํำ

음

ट98 0

$0 \angle 8^{\circ} 0$

$\angle 88^{\circ} 0$

เ9Z' ᄂ

ELC' ।

S82 เ

90E'

0カt・

$\angle S D^{\circ}$ ।

$\nabla \angle t^{\circ}$

$167^{\circ}$

ヤヤト

$\nabla G{ }^{\circ} \varepsilon$
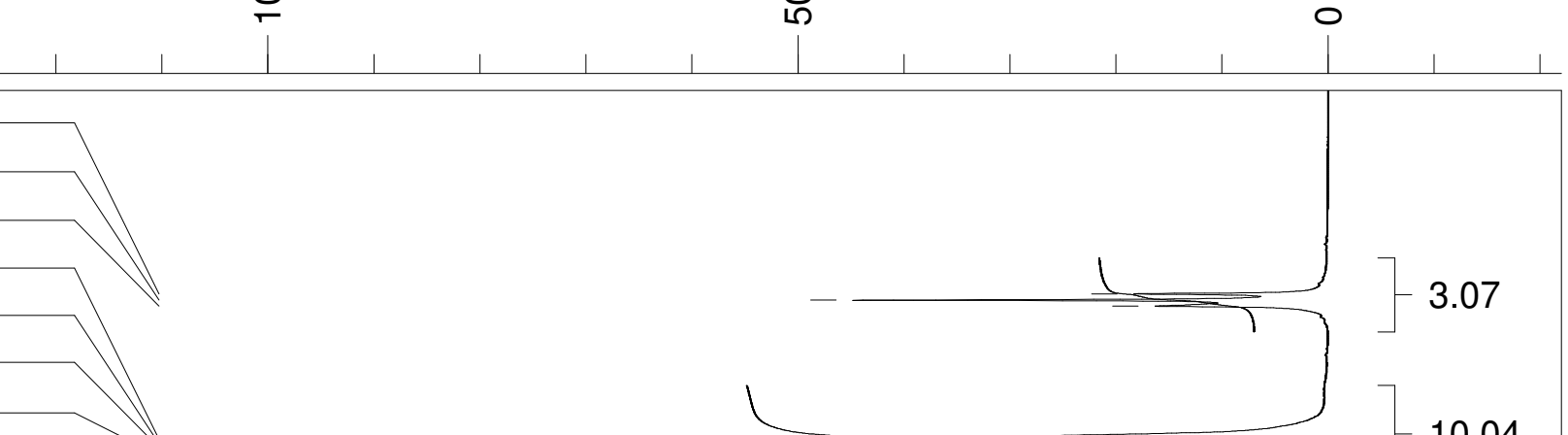

$\longrightarrow$
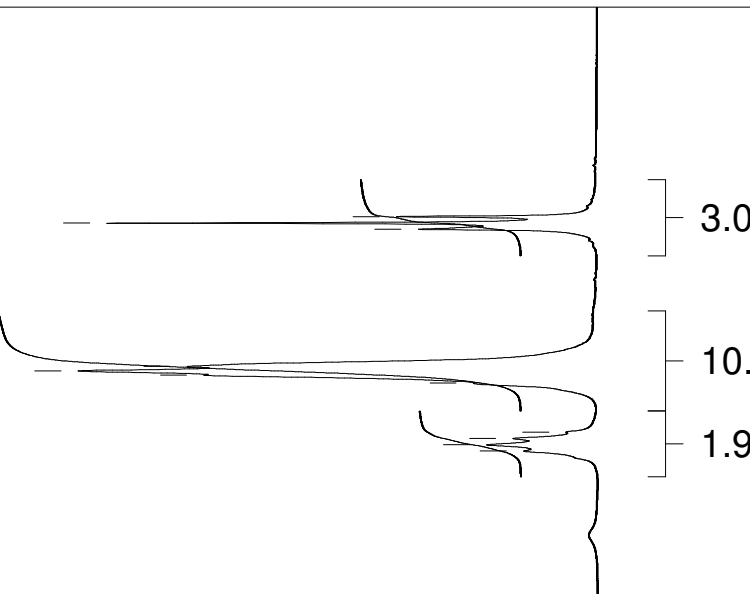


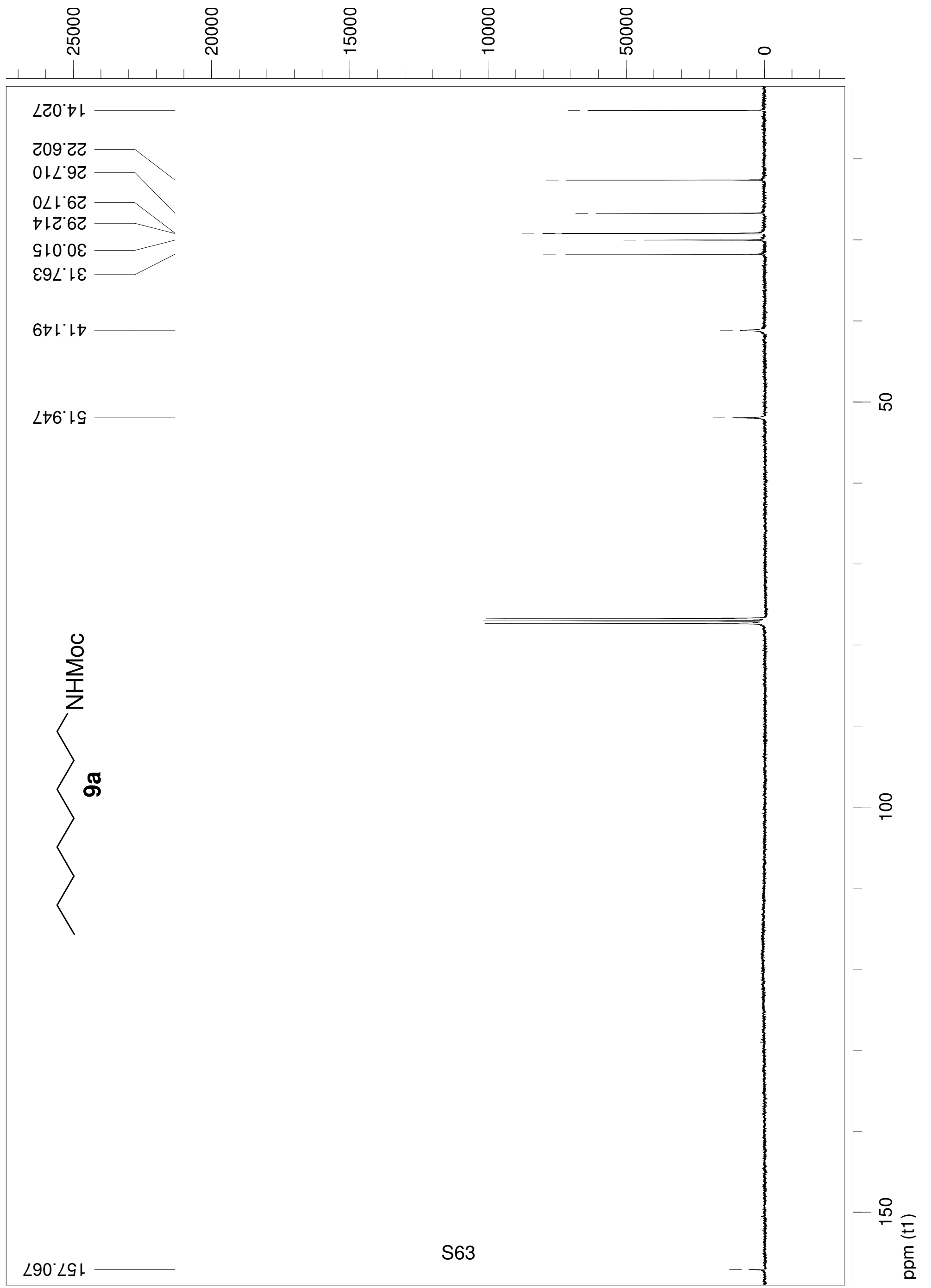


عО乙 $ะ \varepsilon$

86L'IG

S6L'ZG

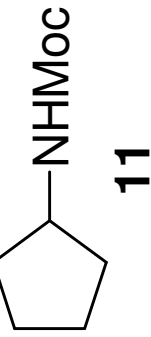



§
๕ ฐ

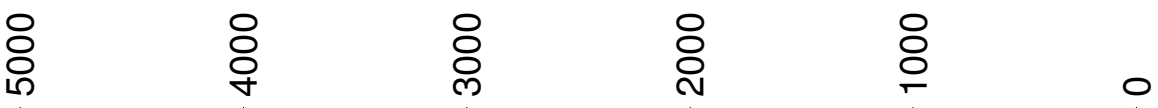
$\begin{array}{llllll}8 & 8 & 8 & 8 & 8 & \\ 8 & 8 & 8 & 8 & 8\end{array}$

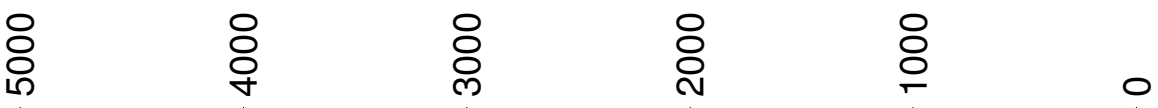

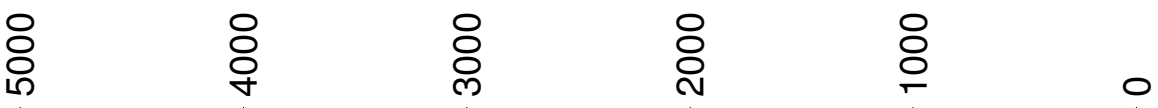
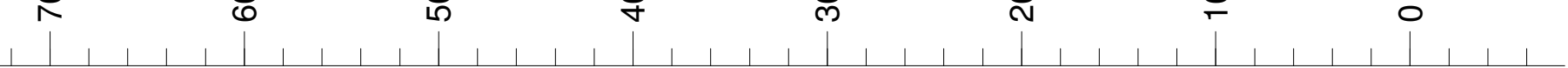

$\triangle \rightarrow 0$

$1 \angle L^{\circ}$

$8 \nabla 2$ เ

$\angle G \varepsilon^{\prime \prime}$ -

EटS" -

269" ।

$\angle E 9^{\circ}$ -

$689^{\circ}$ เ

S98.

†06.

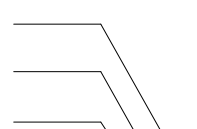




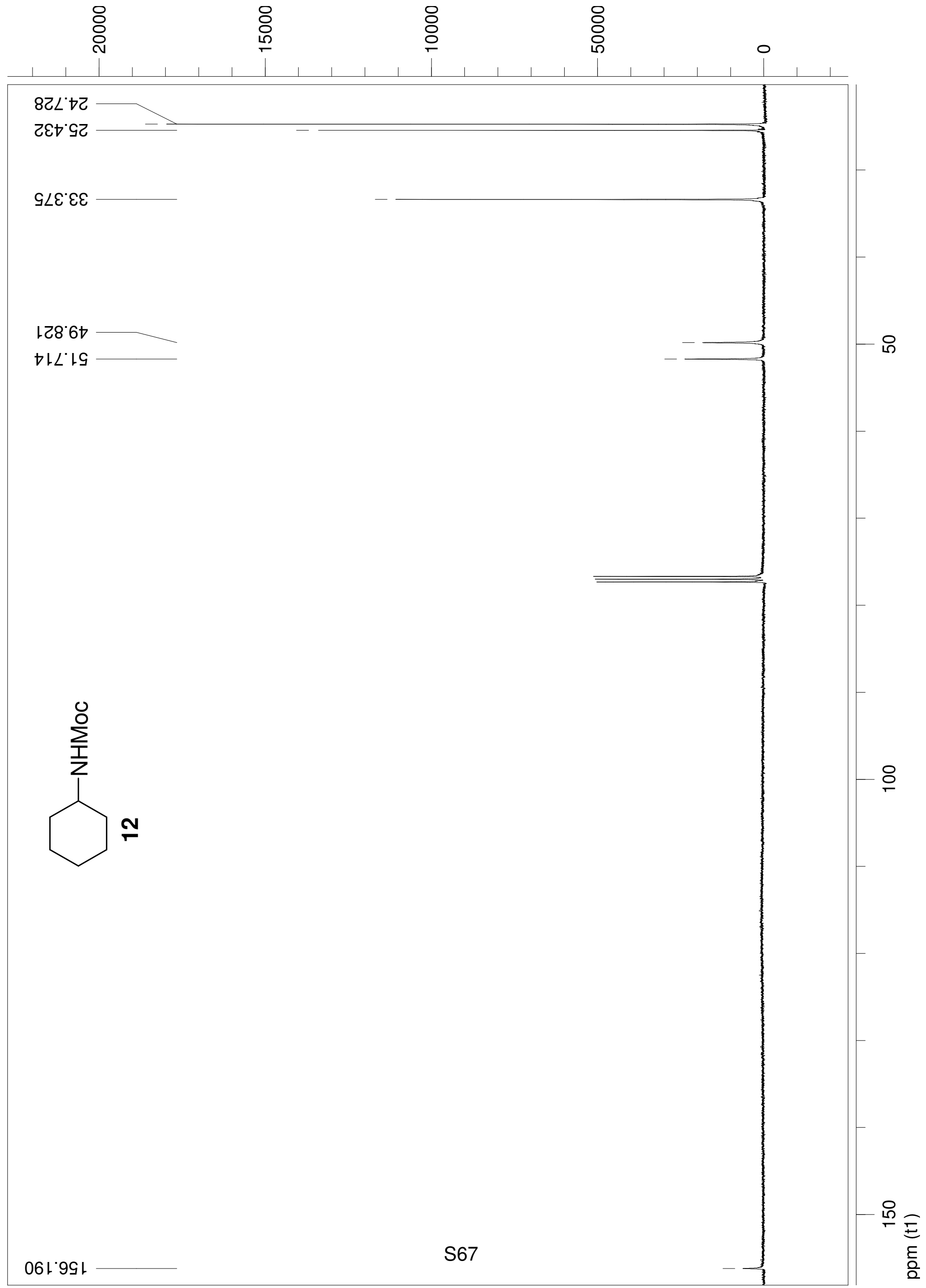




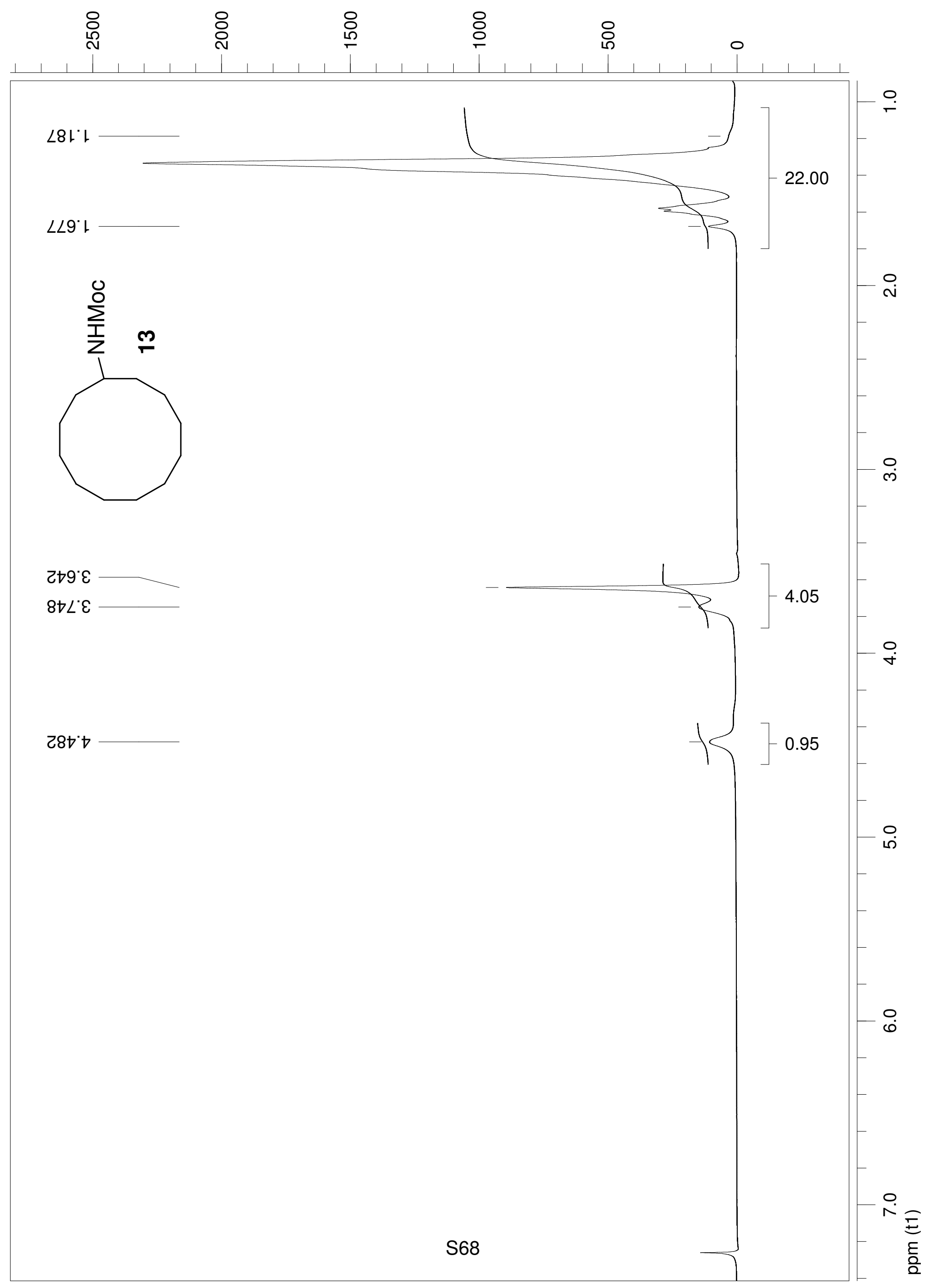




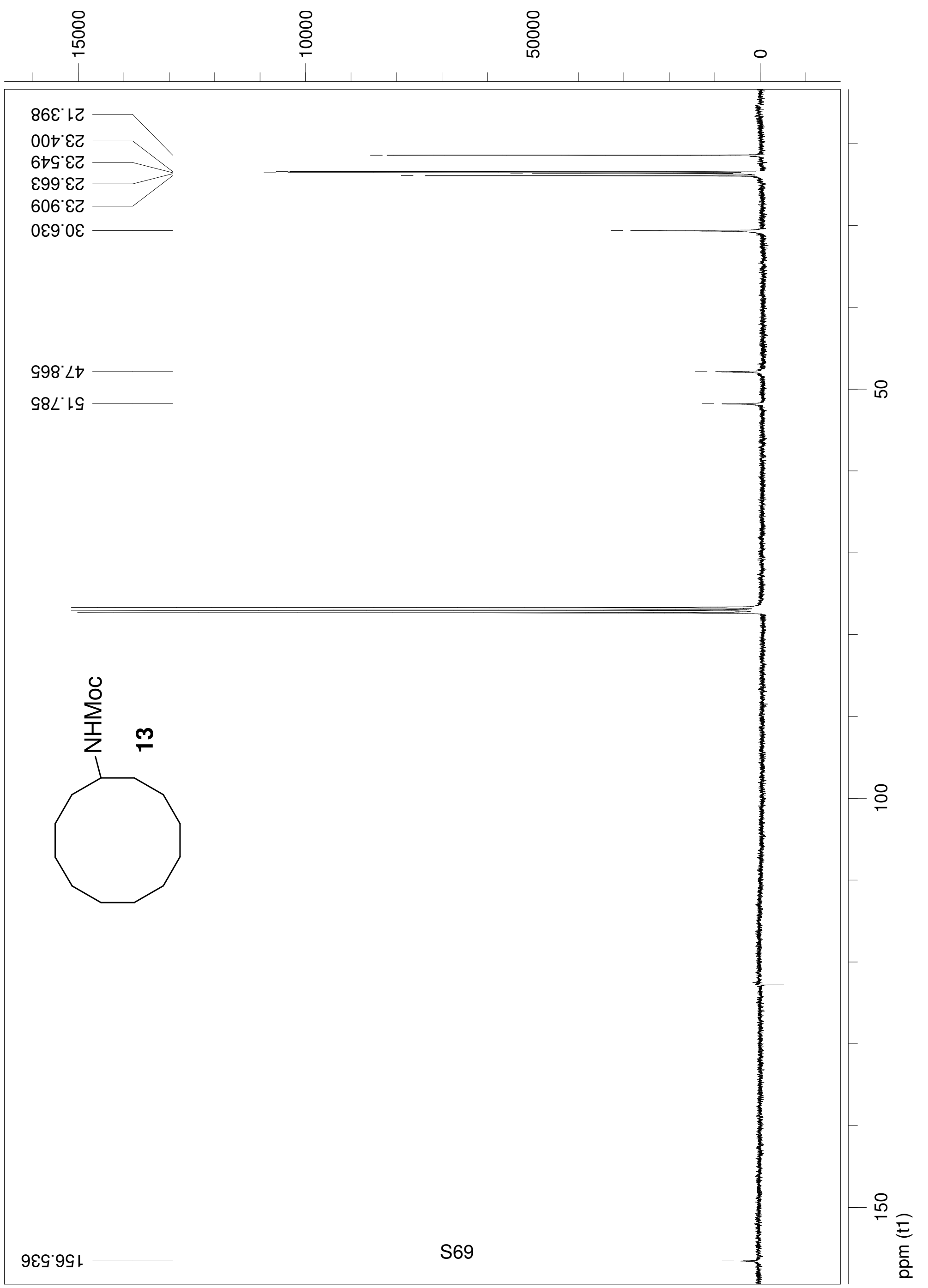




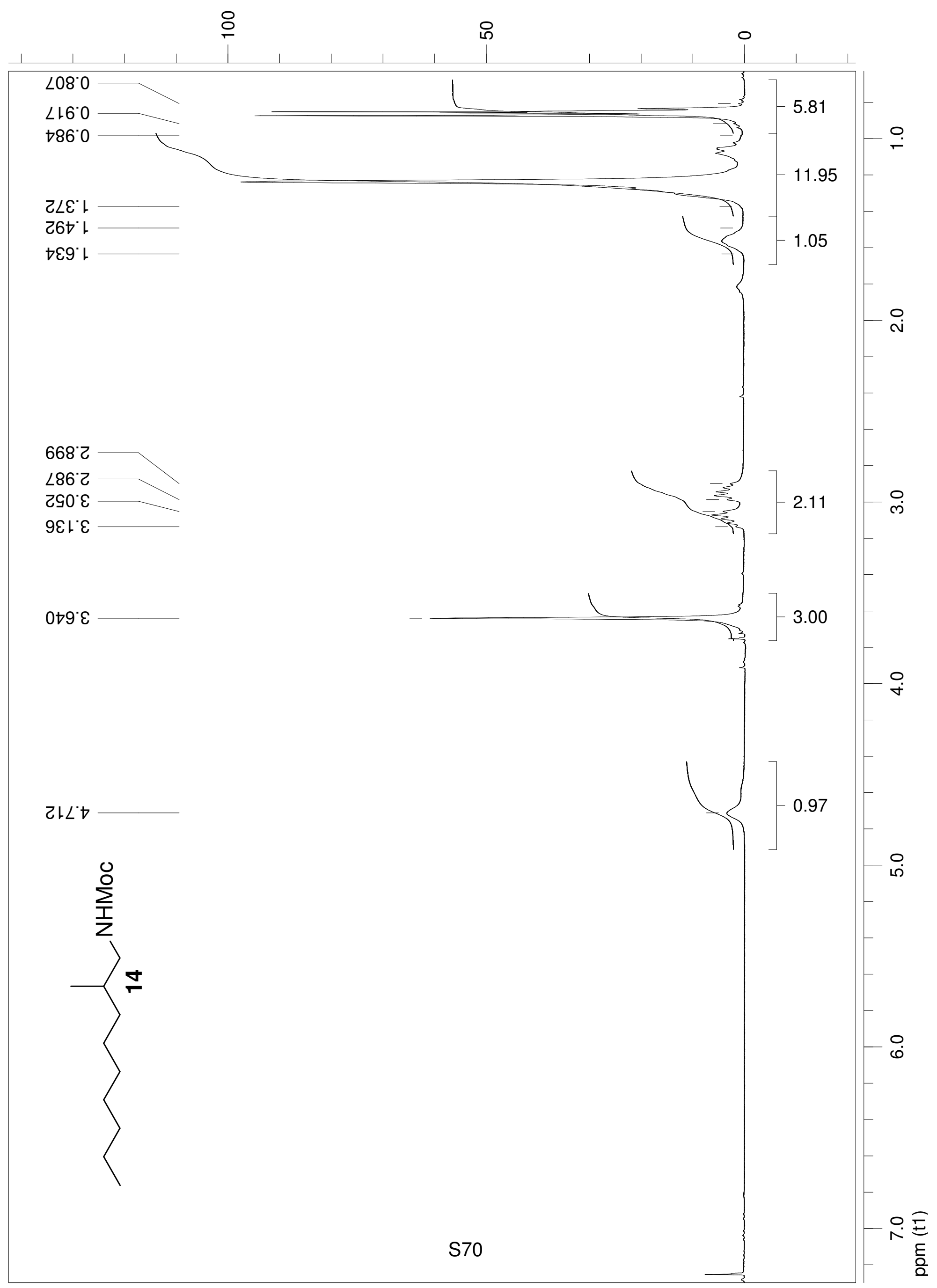




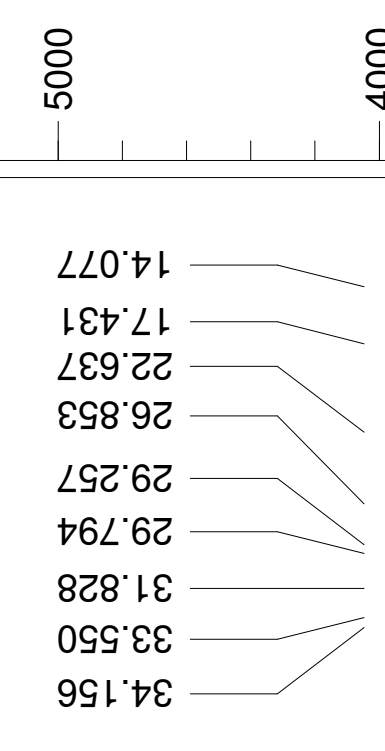

ঃ

옹

음

$890^{\circ} \angle t$

$296^{\circ}$ IG
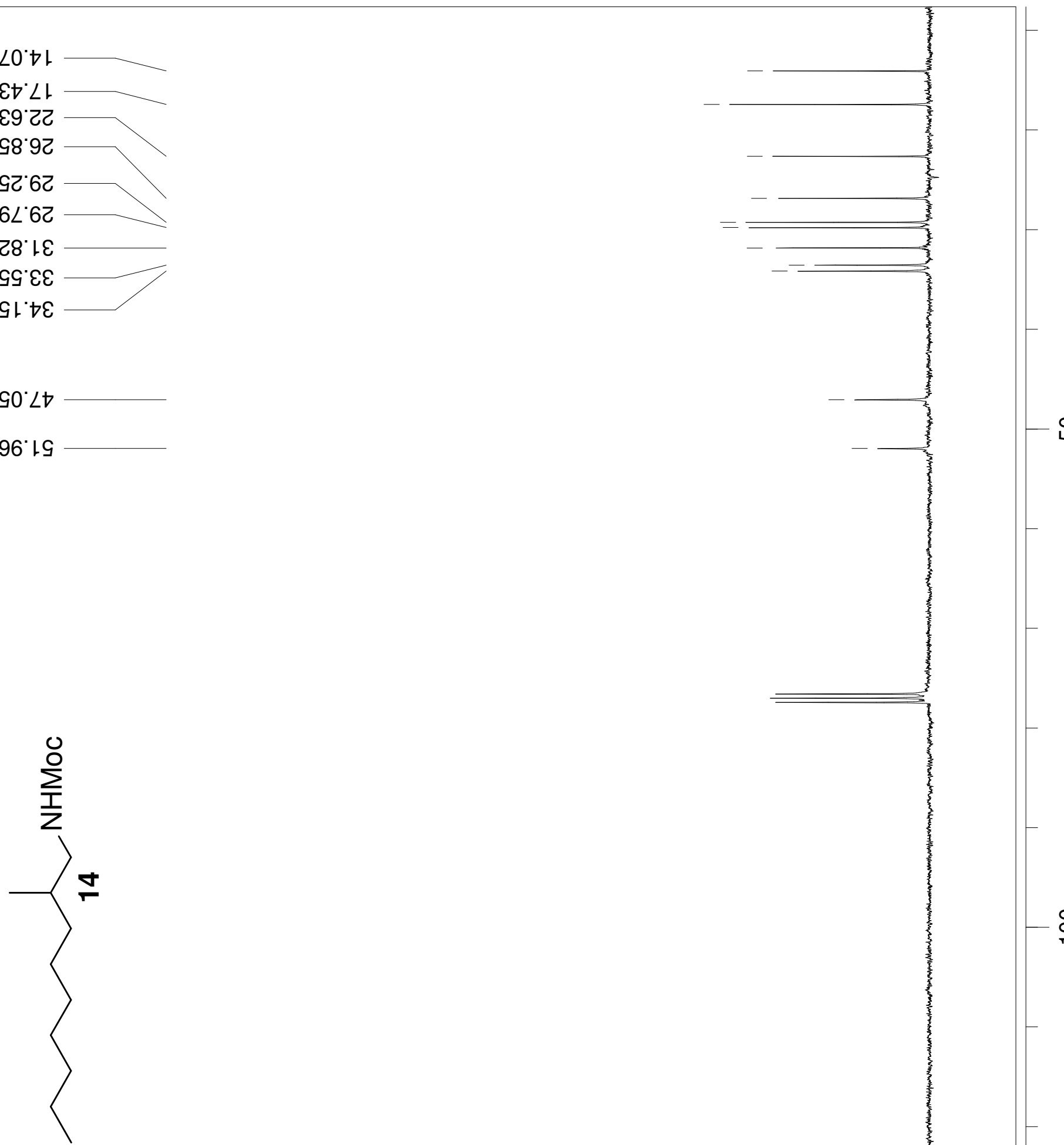


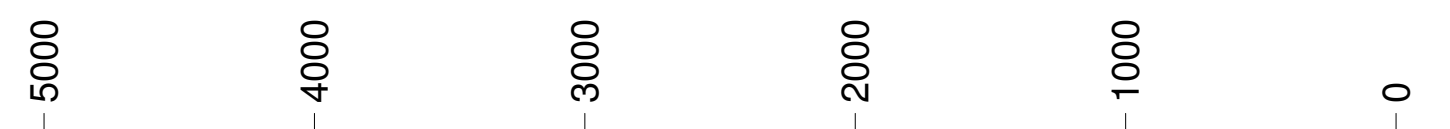

St8.0

ง\&6. 0

$\checkmark \angle 0^{\circ} \mathrm{L}$

$280^{\circ} \mathrm{I}$

992 เ

เトレ・

6เガト

929.

6टL'เ

9ट8 เ

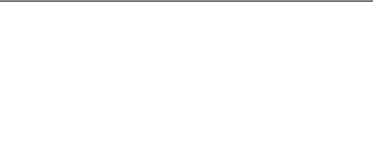

$966^{\circ} 乙$

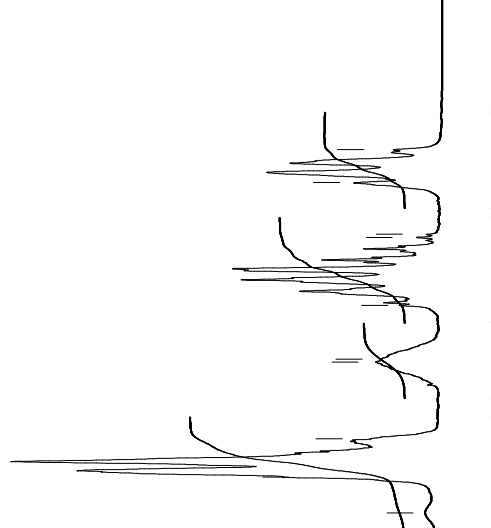

-1.92
-3.00
-0.98
-5.15

$\nabla \downarrow 9^{\circ} \varepsilon$

เレL゙

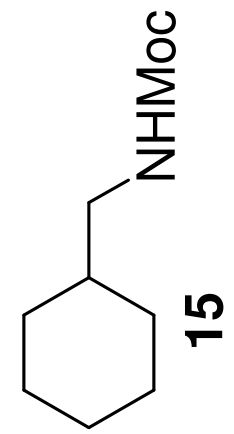




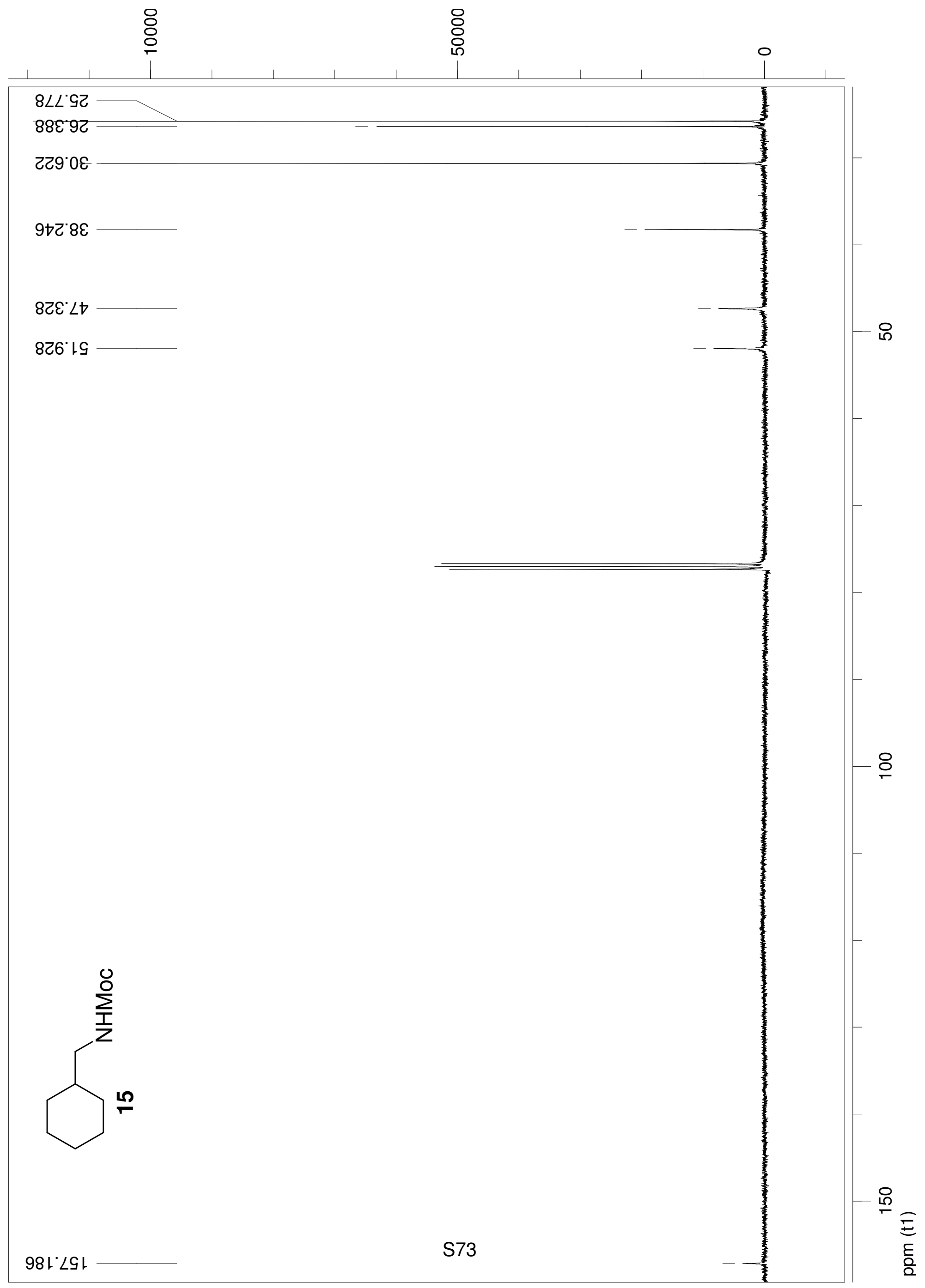




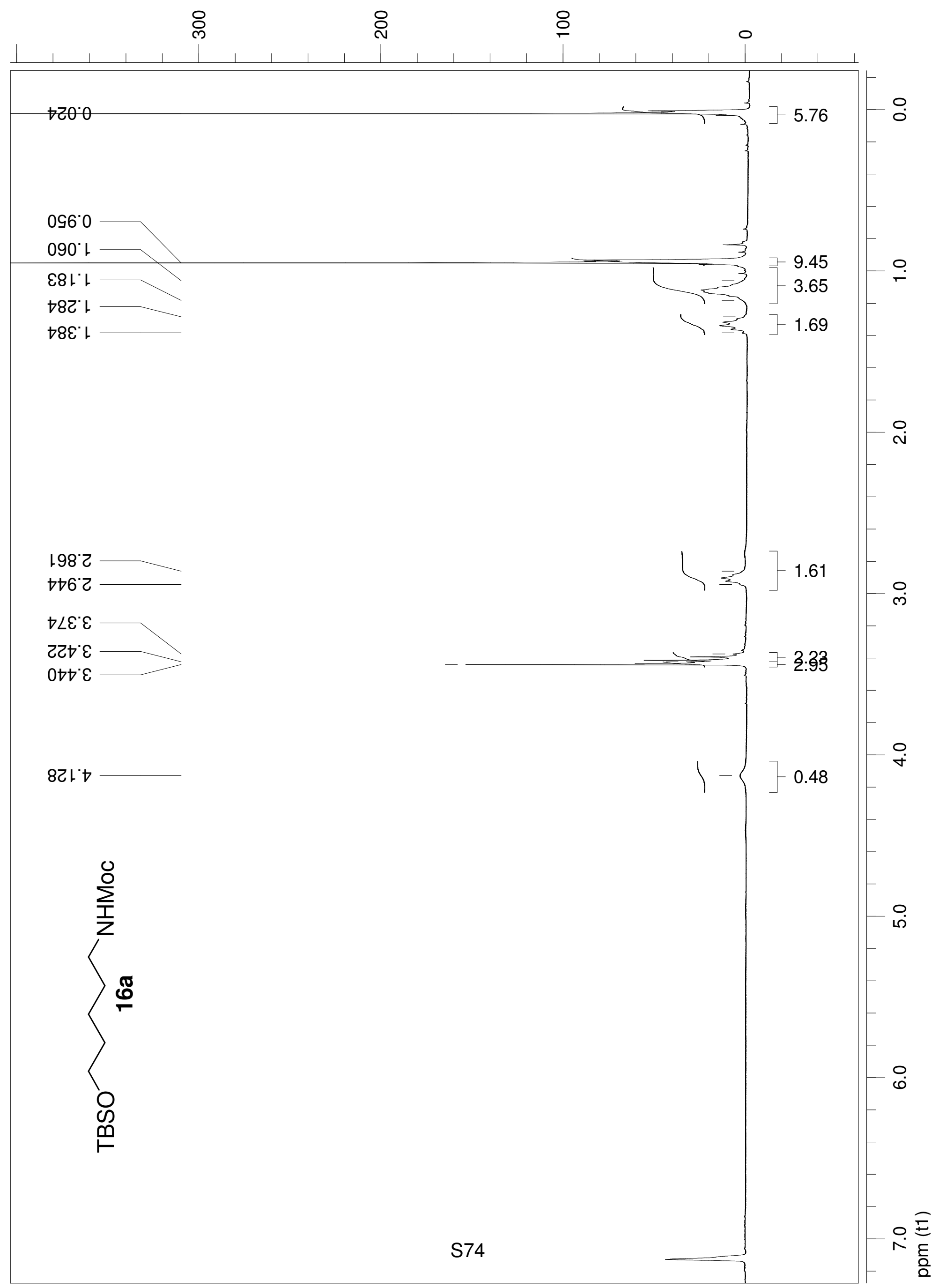




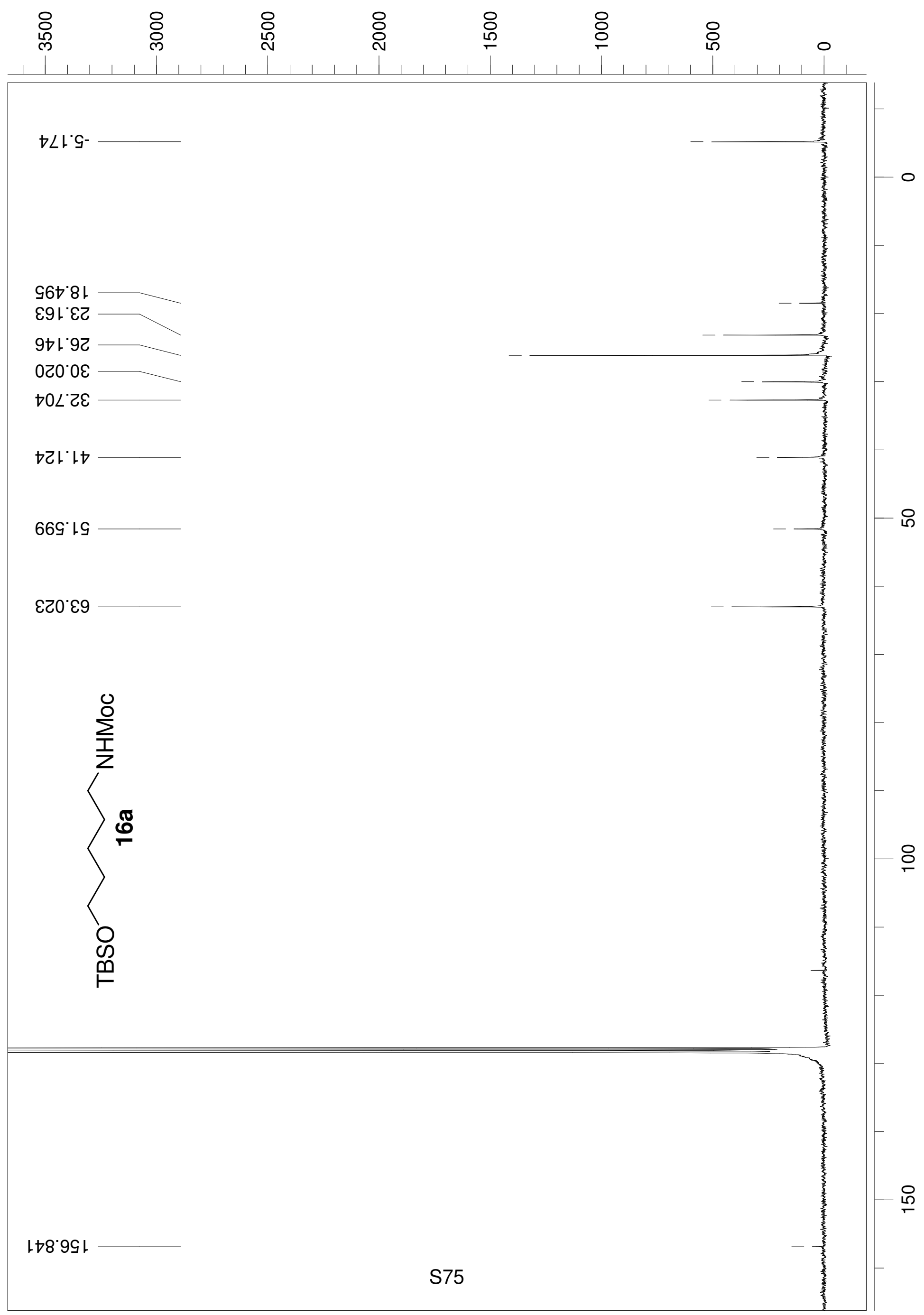




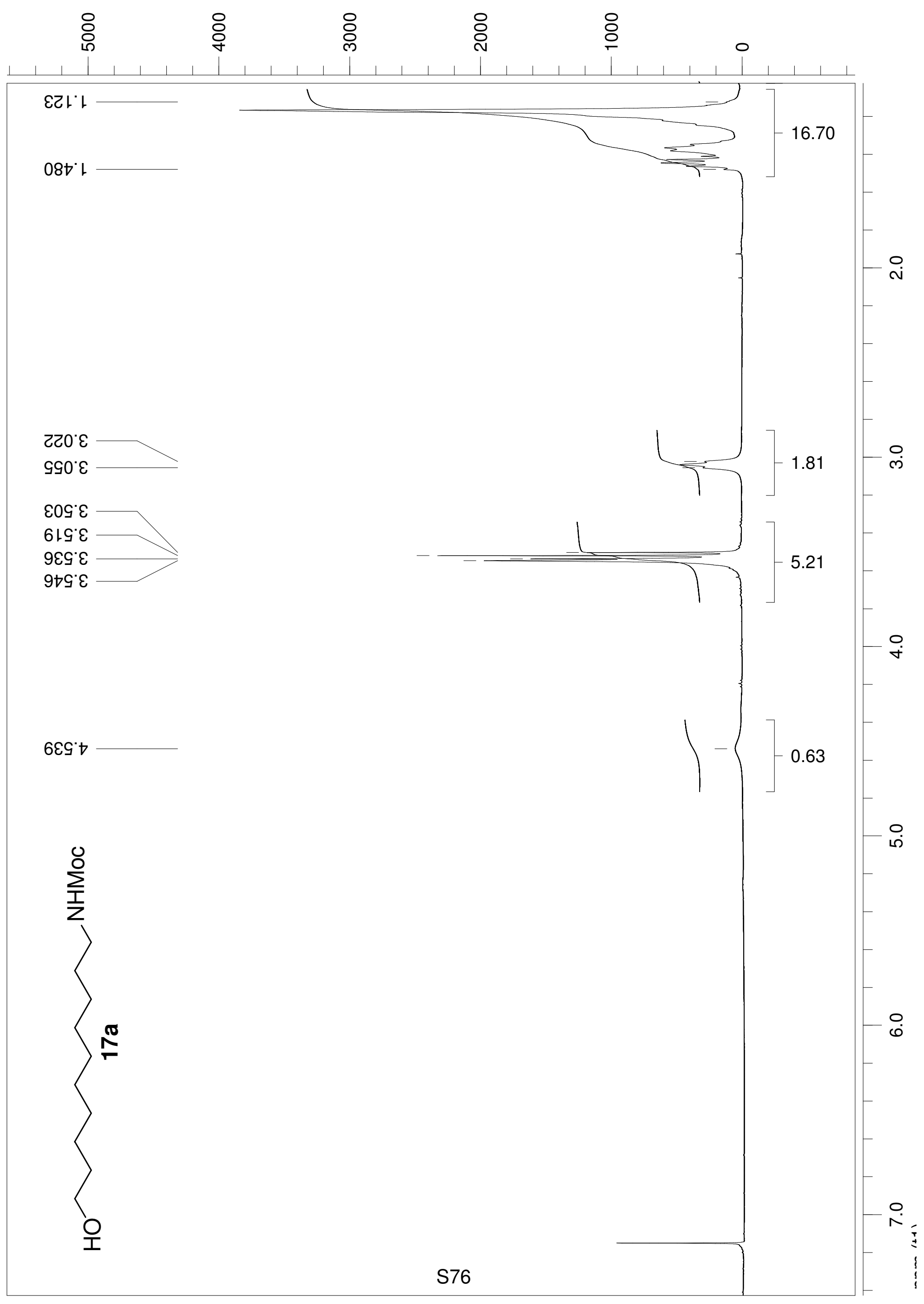




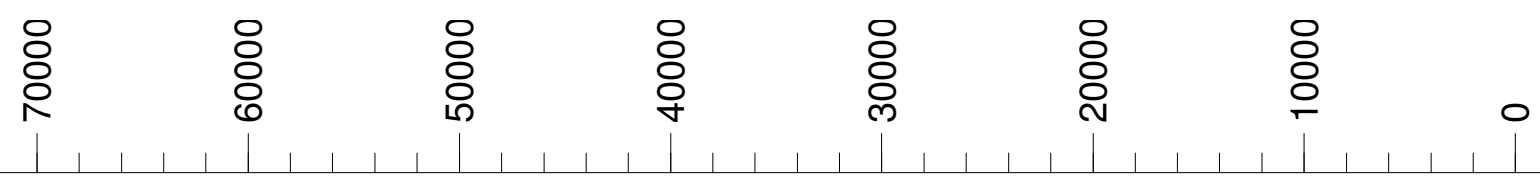

\&69.9ट

ع99.92

$\angle 81^{\circ} 62$

$\varepsilon \varepsilon \varepsilon^{\circ} 62$

เ8ย. 62

เท๋ 62

$266^{\circ} 62$

$6 L L \cdot 2 \varepsilon$

ยトトレナ

S86.'

เ२० \&9

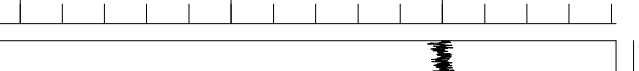

120.89

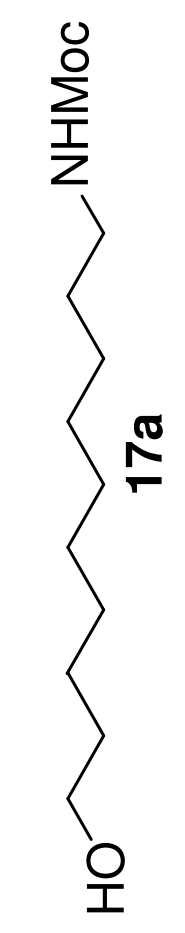


$6 \varepsilon 0^{\circ} 0$

ट88 0

$806^{\circ} 0$

गट6 0

$1 \nabla 6^{\circ} 0$

†Z8' ᄂ

$126^{\circ}$

웅

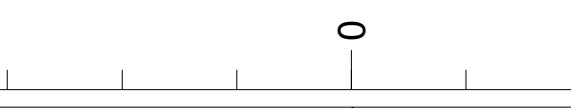

$60 \nabla^{\circ} \varepsilon$

$69 \mathcal{G}^{\circ} \varepsilon$

$9 \mathcal{G}^{\circ} \varepsilon$

$6 \varepsilon 9^{\circ} \varepsilon$

เ $L^{\prime} \varepsilon$
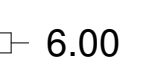

웅

$-15.48$

음

- 1.06

ำ

$028^{\circ}$

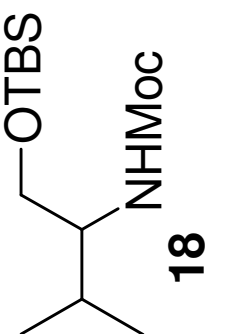




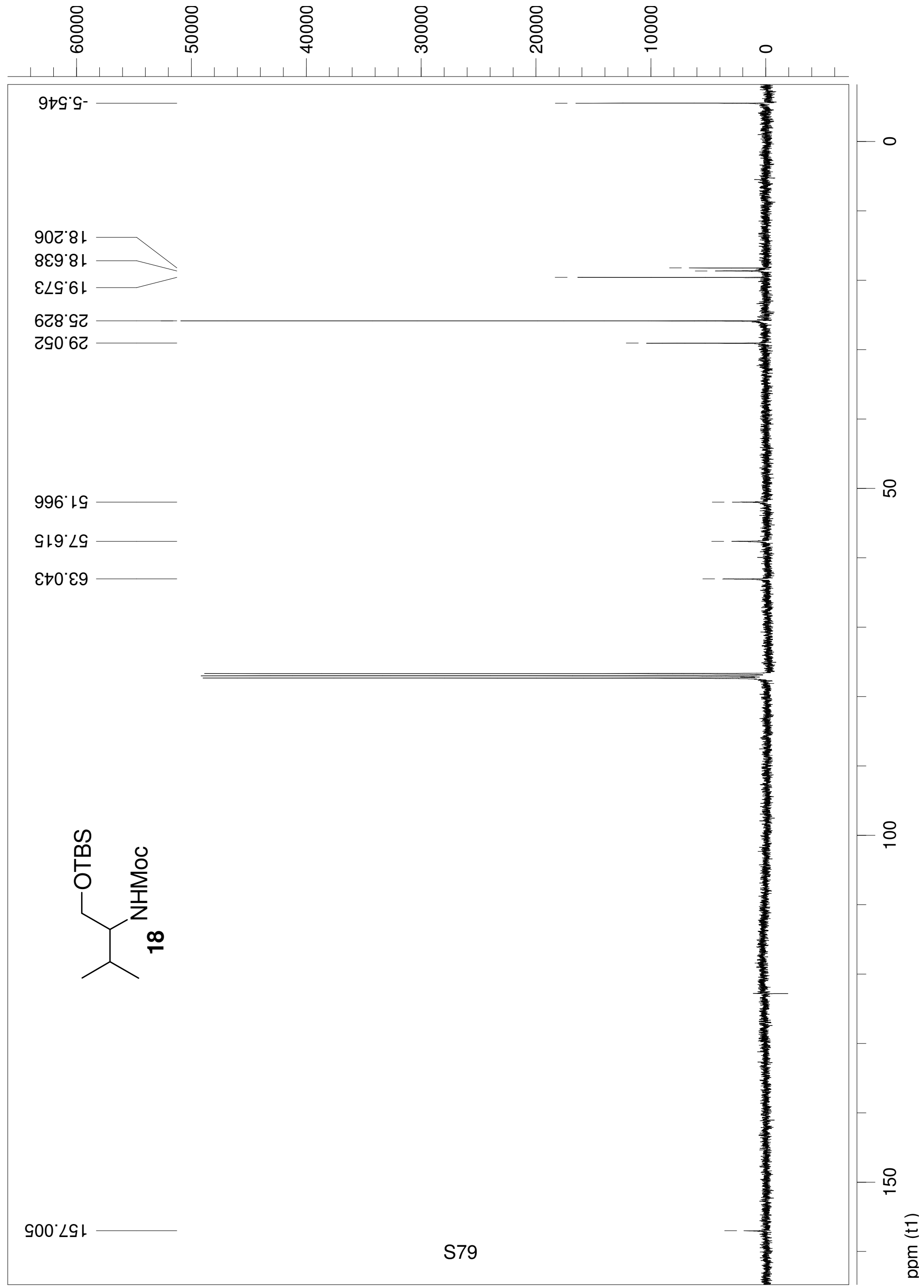


ষ

\&

ㅇำ

$\stackrel{8}{\circ}$

ZLL'

$76 L^{\circ} \cdot$

Sเ8.

$9 \varepsilon 8^{\circ}$ เ

$898^{\circ}$ เ

$0+00^{\circ} \mathrm{C}$

$90 z^{\prime} \varepsilon$

9टว $\varepsilon$

$8 \nabla \tau^{\prime} \varepsilon$

$89 \mathcal{2}^{\circ} \varepsilon$

$0 \multimap 9^{\circ} \varepsilon$

$\nabla 60^{\circ} t$

カルド

sel't

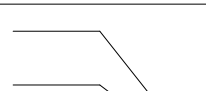

(n)

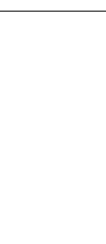

-

疋
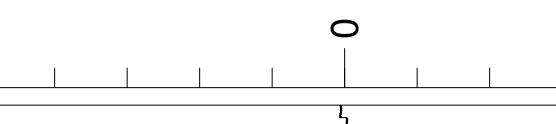
음

‡

웅

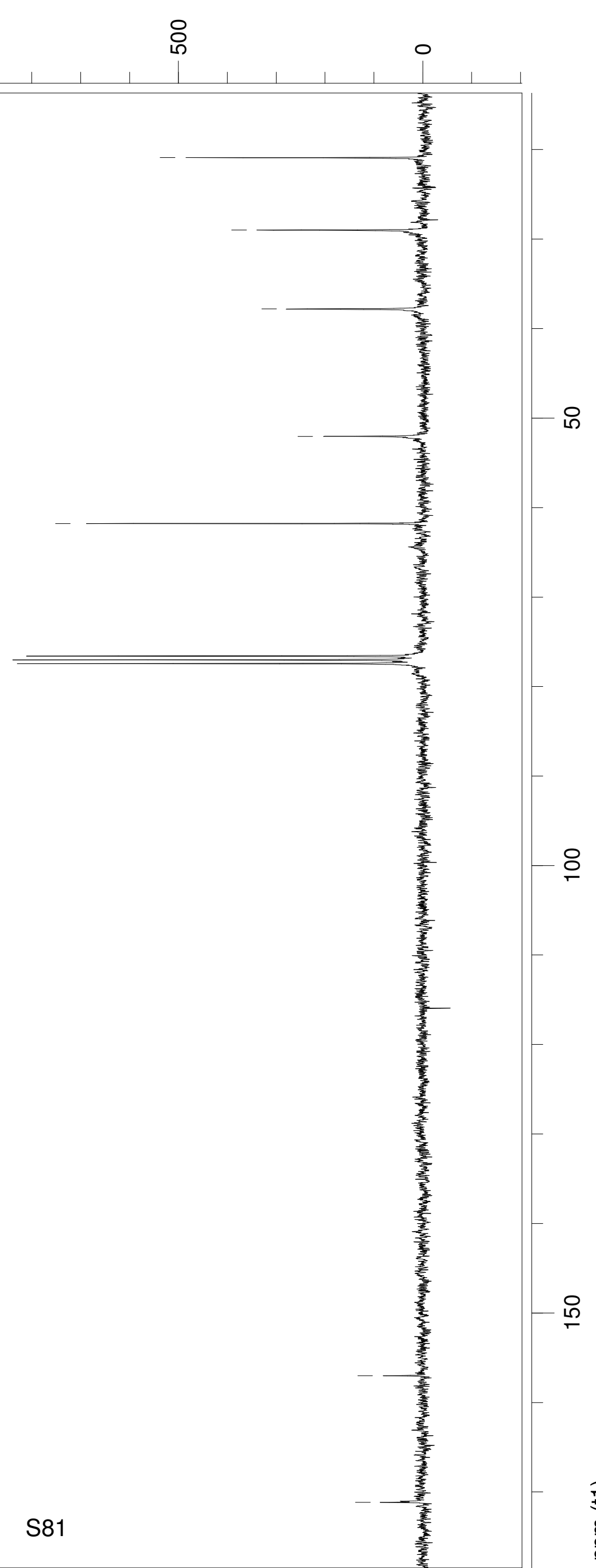

E
$\frac{\text { 을 }}{2}$ 


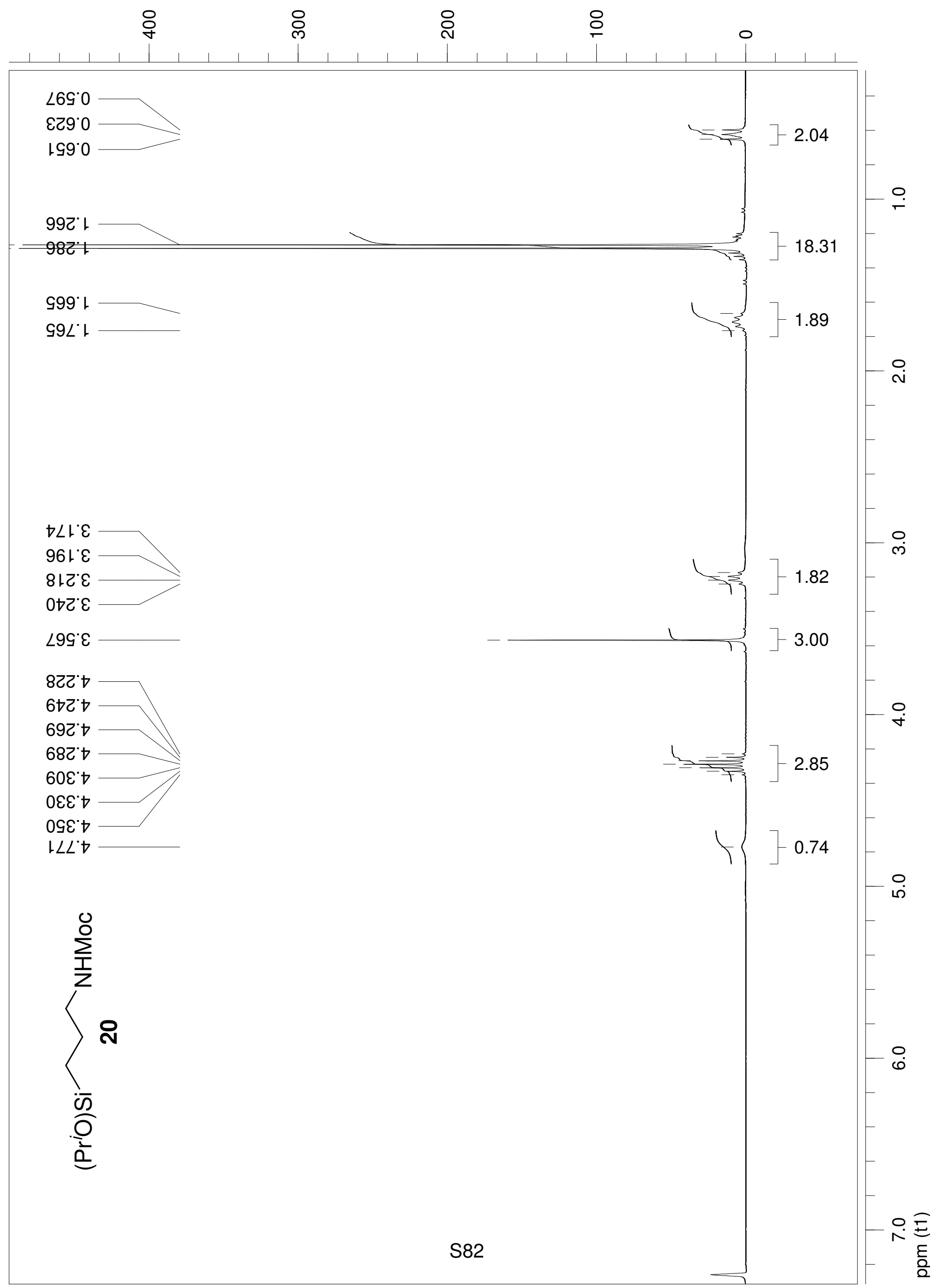




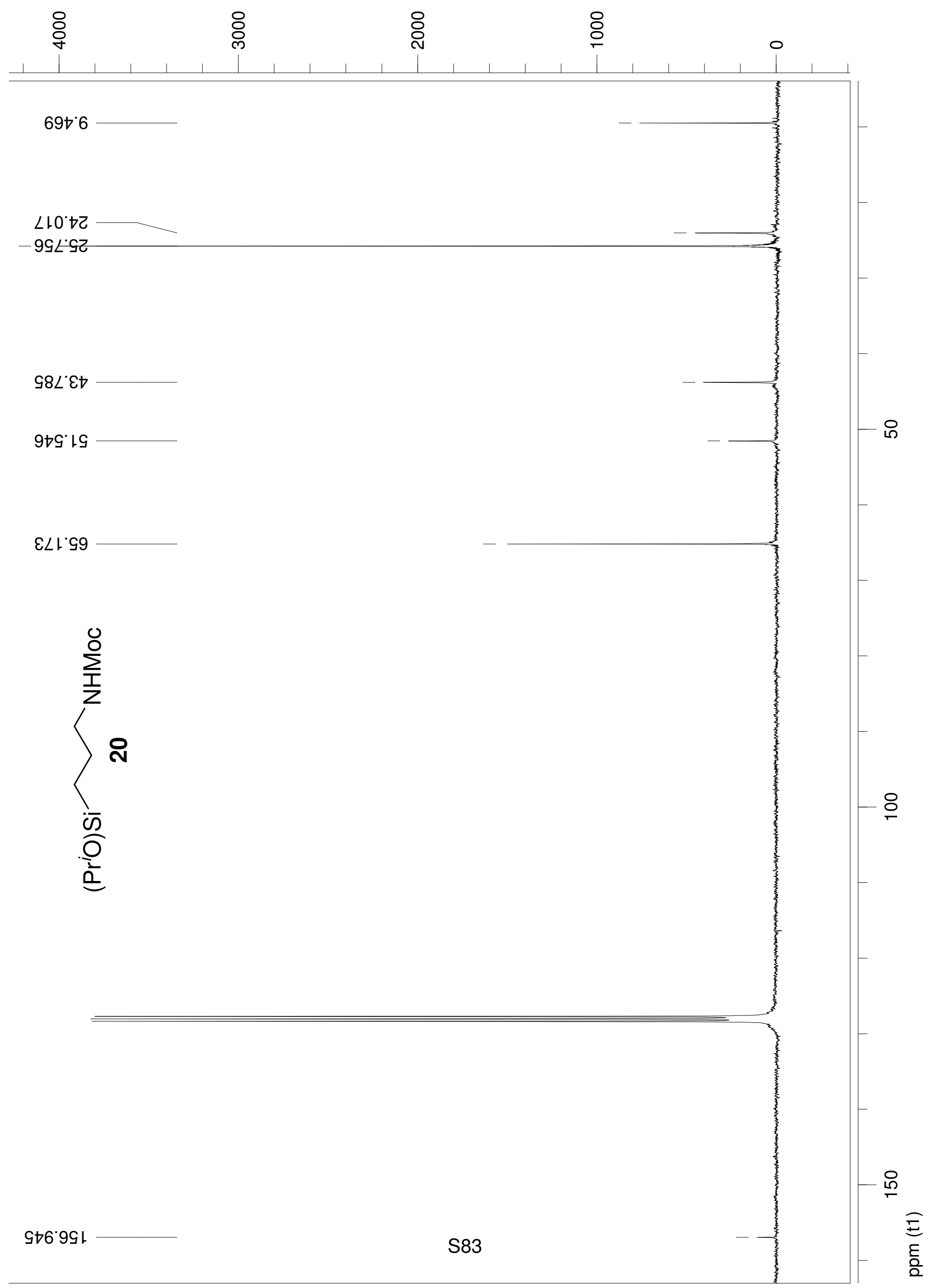


ㄴㄴ

† $98^{\circ}$

8टح $\varepsilon$

เ๑9 $\varepsilon$

SOL' $\varepsilon$

$\overline{\tau \varepsilon L^{\prime} \varepsilon}$

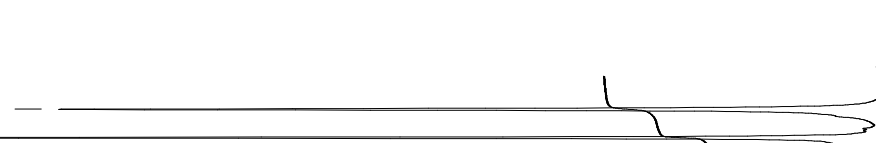

$601^{\circ} 9$

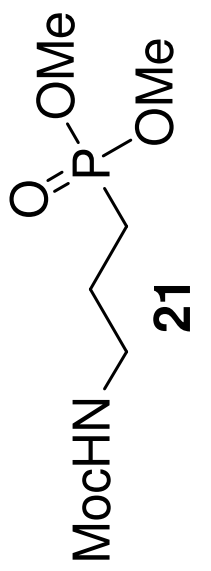




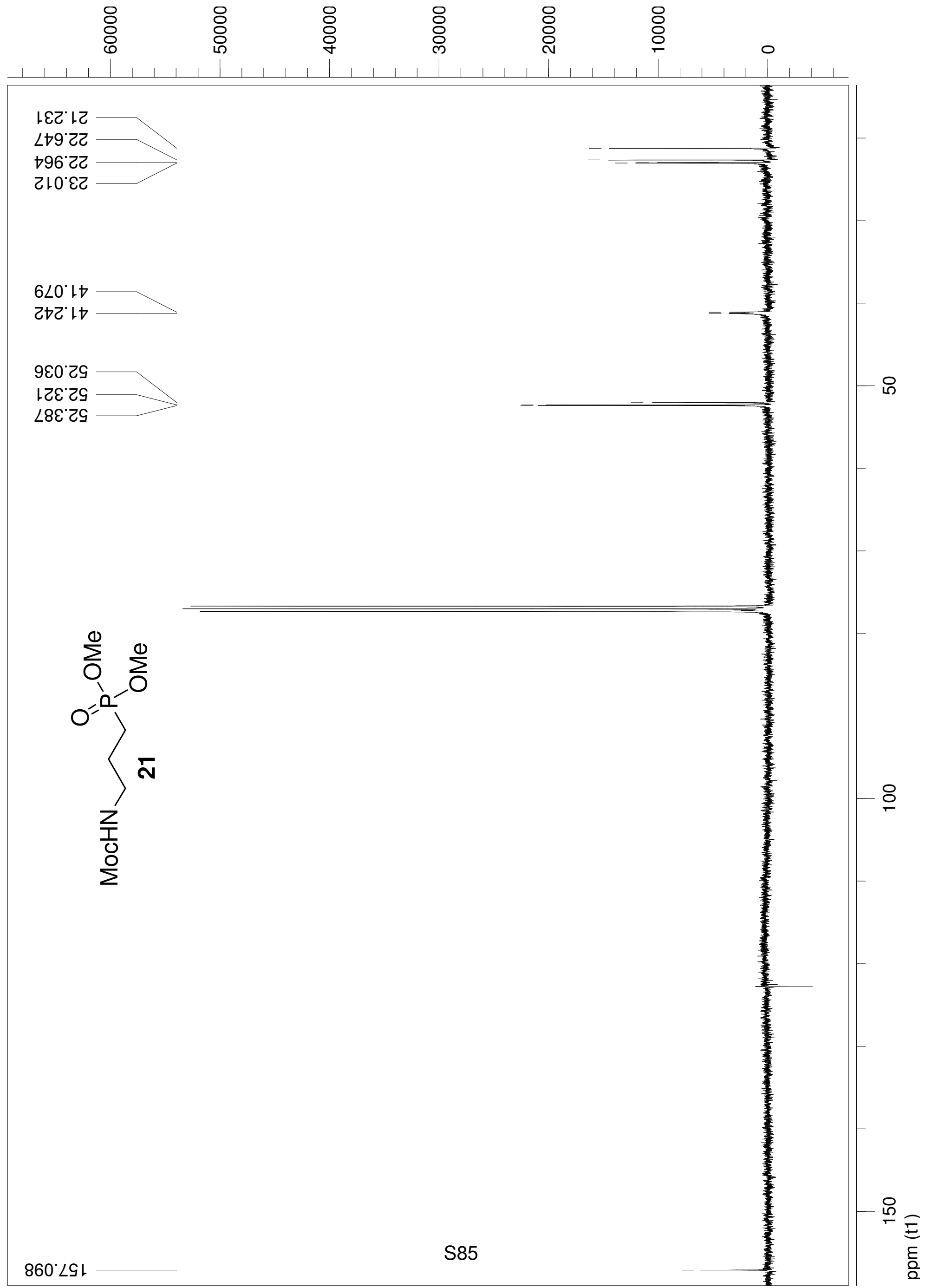




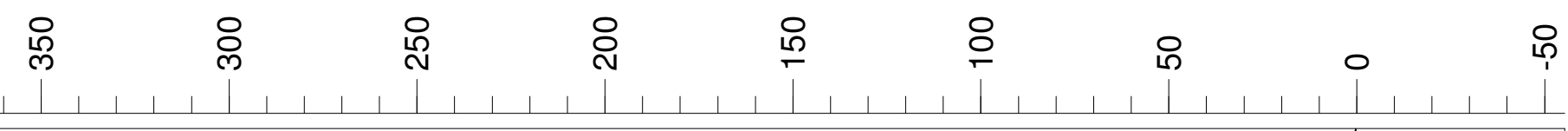

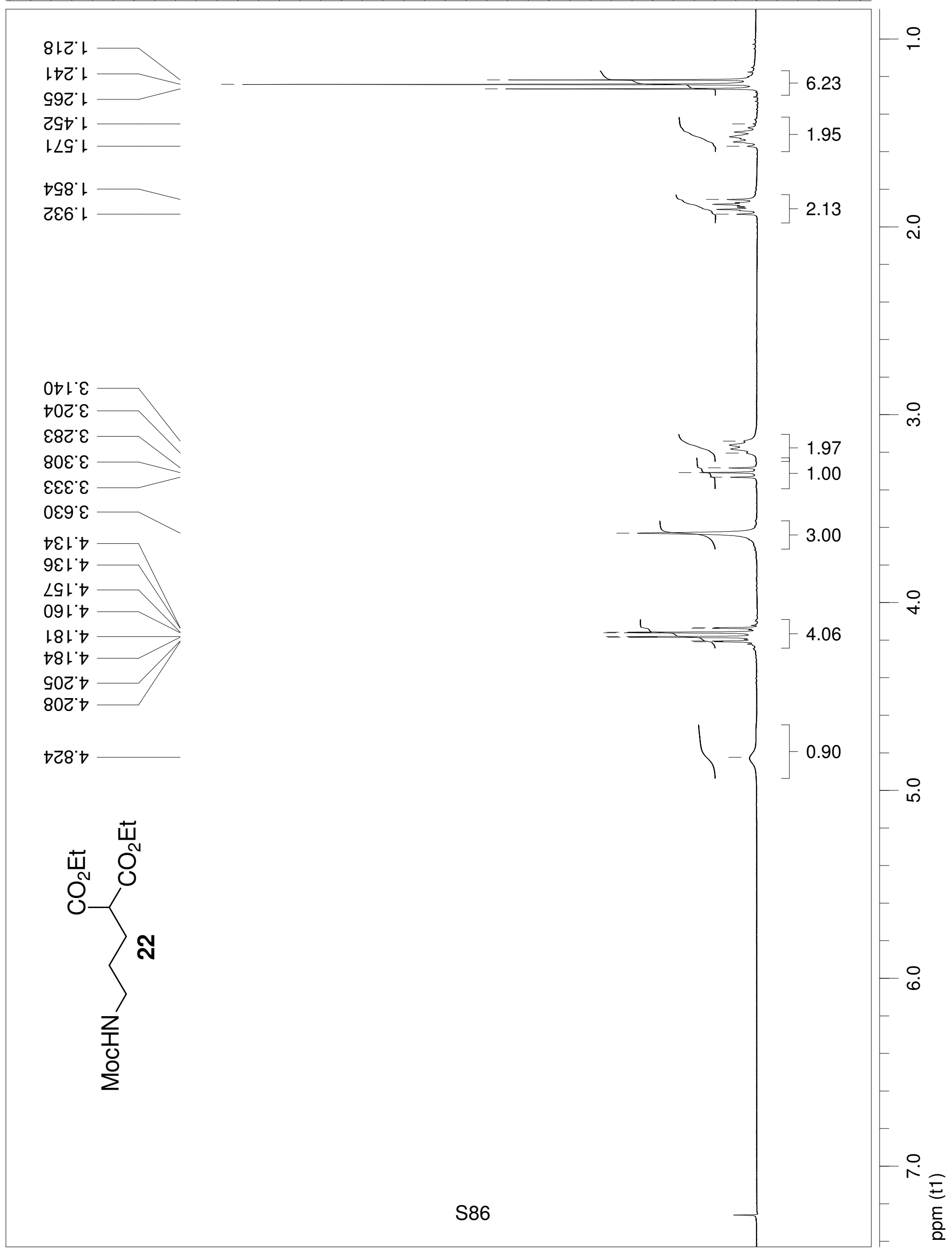




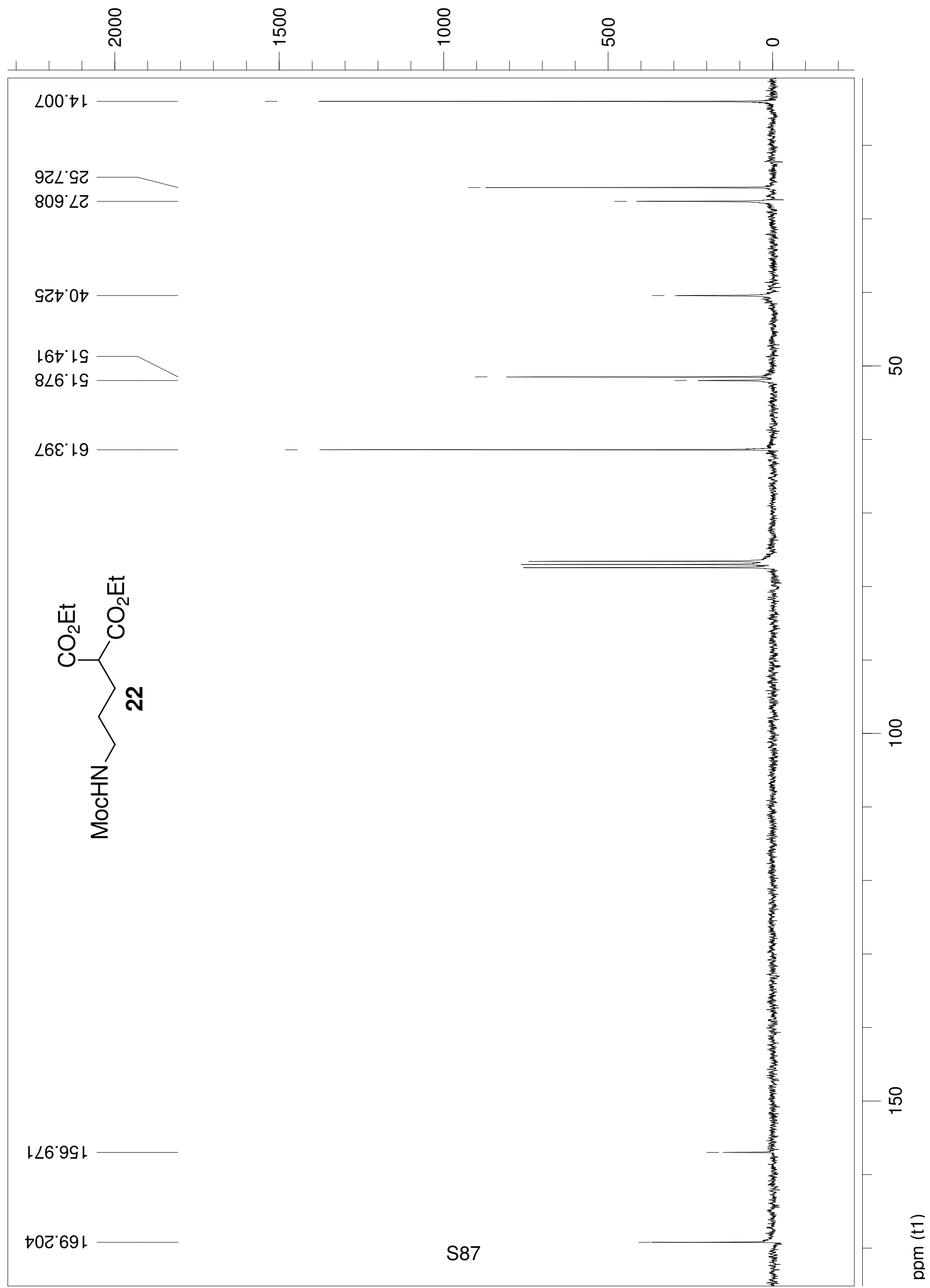




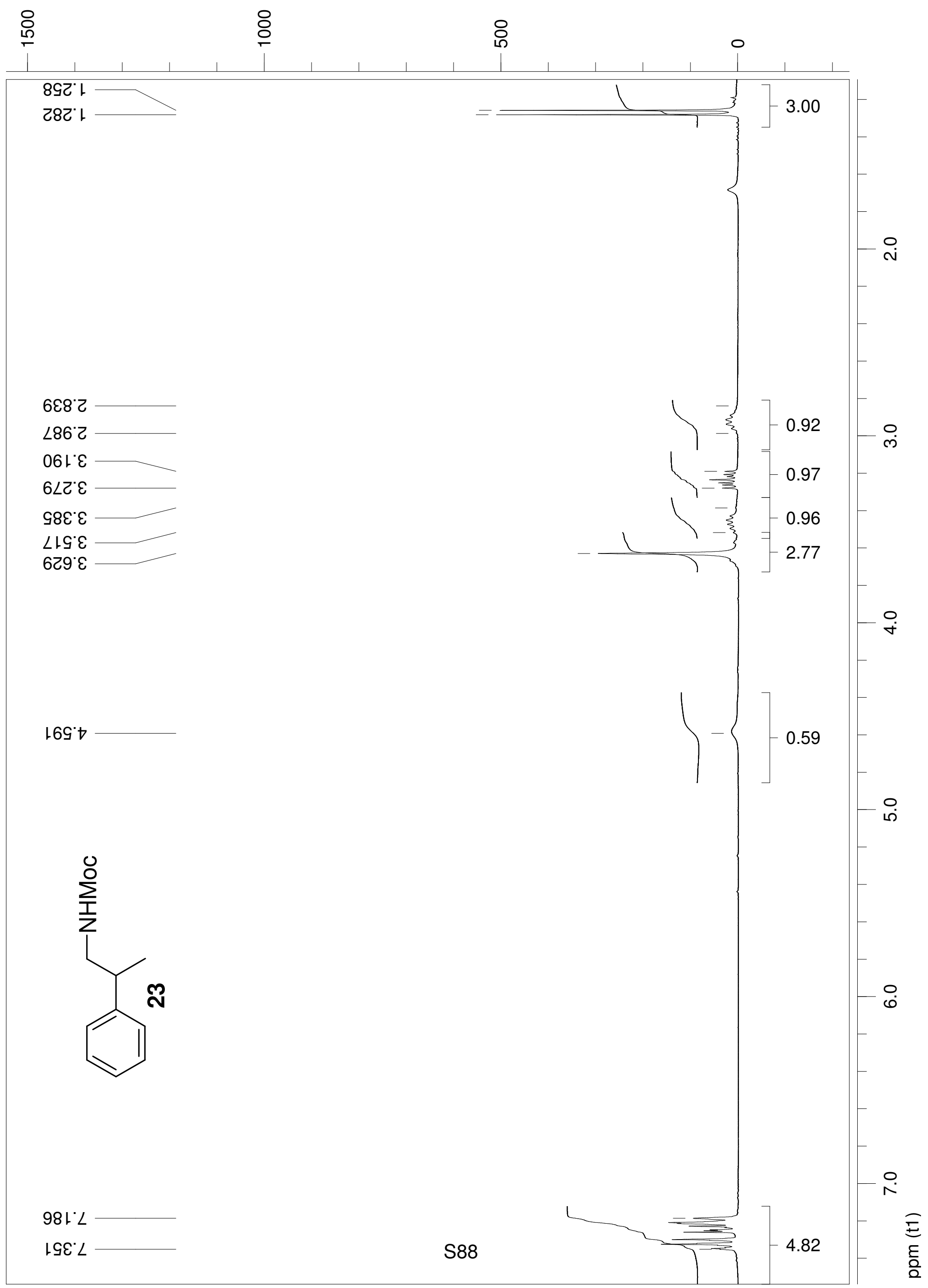


\&

$760^{\circ} 6$ เ

乙†0 $0 t$

$769^{\circ} \angle \nabla$

600 '29

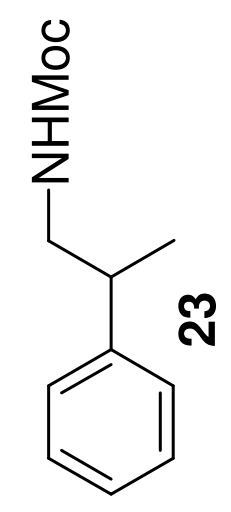

699.92

GSI'LCL

8†9.8ट।

เレ6゚๖เ 
웅

음

유

응

9टเ०0-

टट०० 0

$916^{\circ} 0$

Еट० $\varepsilon$

$9 \varepsilon{ }^{\circ} \varepsilon$

$0 \varepsilon \varepsilon^{\circ} \varepsilon$

$66 \varepsilon^{\circ} \varepsilon$

ยจฑ $\varepsilon$

$6 \angle 9^{\circ} \nabla$

ISL'D

$16 L^{\circ} t$<smiles>[C]CC(O)c1ccccc1</smiles>

$600^{\circ} \mathrm{L}$

$62 L^{\circ} \mathrm{L}$

$68 \mathrm{I}^{\circ} \mathrm{L}$

$\angle E Z \angle$ 


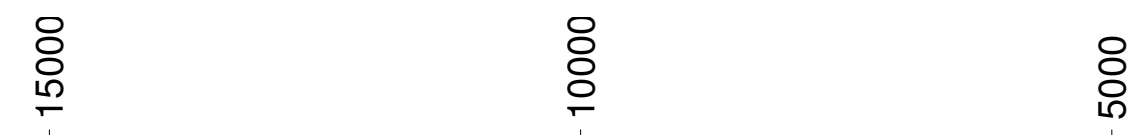

0عเ'

$1 \angle L{ }^{\prime} D^{-}$

tGเ'8เ

$29 \angle \mathrm{AL}$

$19 \varepsilon^{\circ} 6 t$

เ60'乙S

$\mathrm{S}^{\circ} \varepsilon L$

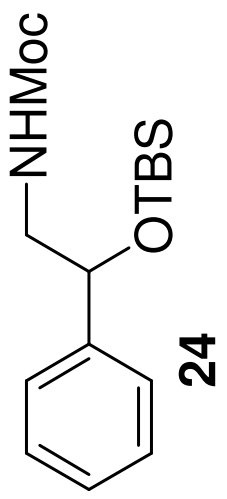

S96.9ट।

6SG $\angle 2 L$

५Еट 8ट

くトレでト

986.99 
90 เ 0 -

$8 \varepsilon 0^{\circ} 0$

$\angle 88^{\circ} 0$

SOL $\mathcal{~} \varepsilon$

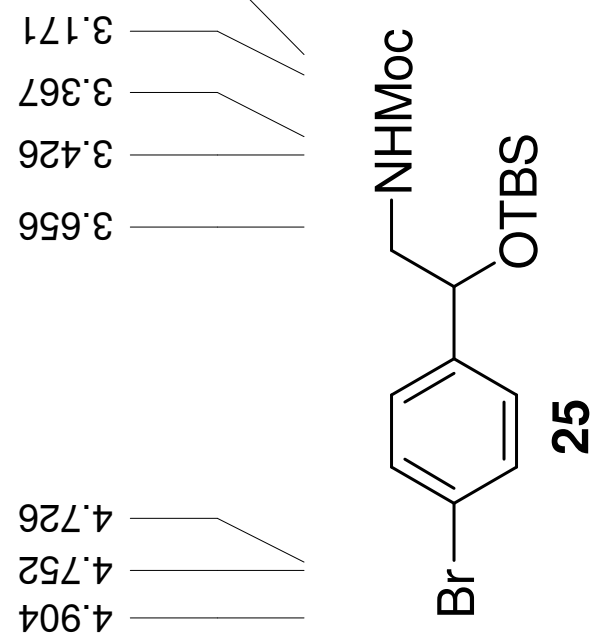


量 高 高

crty

$\angle L L^{\prime} D^{\circ}$

५Eเ 8เ

$6 \nabla L \cdot 92$

$\angle L Z^{\prime} 67$

EEเ'ZS

$69 L^{\circ} \varepsilon L$

$\varepsilon 89^{\circ} 9 L$

$000^{\circ} \angle L$

$81 \varepsilon^{\circ} L L$

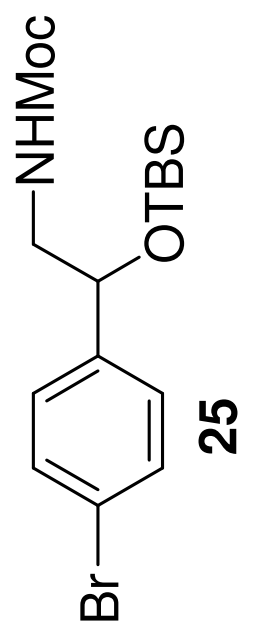

૬こオ゙เてト

G69 LCL

0ยฑ เEเ

こ6でレレ

L96.991 


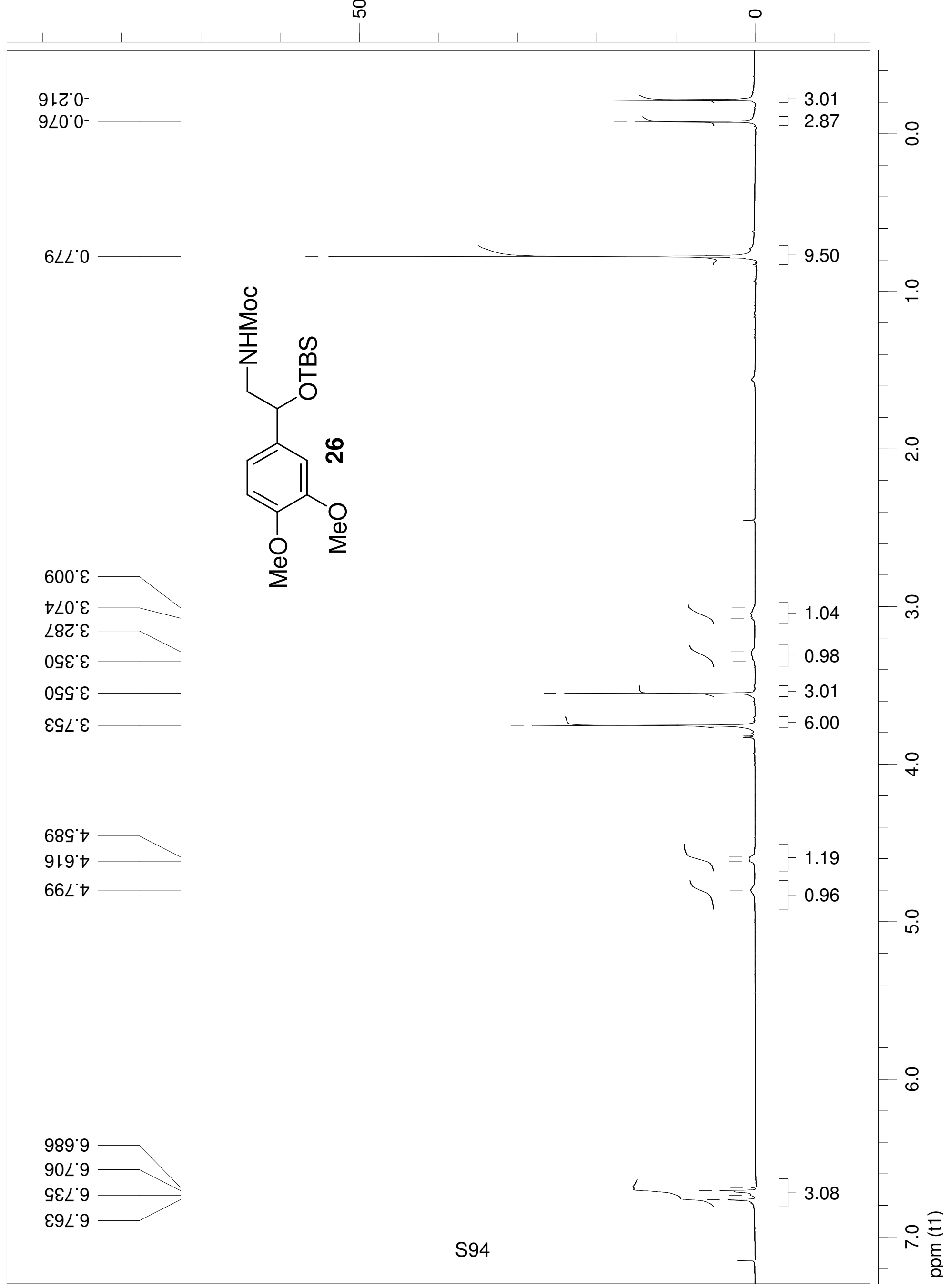



高
ঃ
용
옹
N

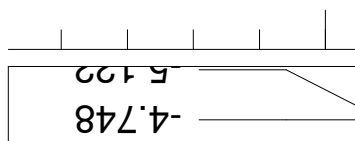

8ヤL゙ち

LGI'8L

$\angle G L G Z$

เSt 67

$\triangleright \triangleright 0$ ¿G

\&08.99

ट९8.99

66ฑ $\varepsilon L$

乙 $\angle 0^{\circ} 60 \mathrm{~L}$

968안

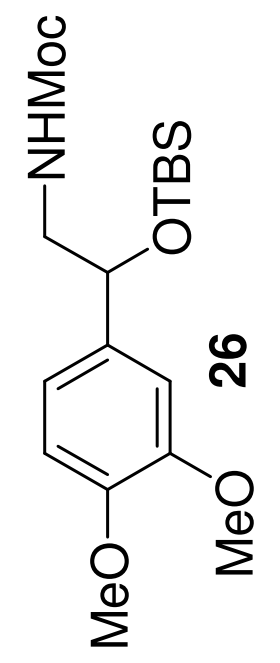

96เ゚ロレ

698" $\downarrow \varepsilon \vdash$

$\angle 8 \varepsilon^{\circ} 8$ เ

968:ㄲ

SL6.99L 

气

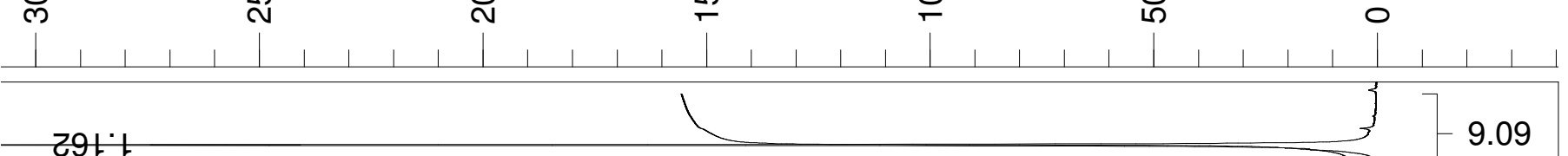
z91

เ6乙' $\varepsilon$

$\varepsilon 0 \varepsilon^{\prime} \varepsilon$

SเE' $\varepsilon$

$68 \varepsilon^{\circ} \varepsilon$

ट०० $\varepsilon$

sเt' $\varepsilon$

$999^{\circ} \varepsilon$

¿\&०॰

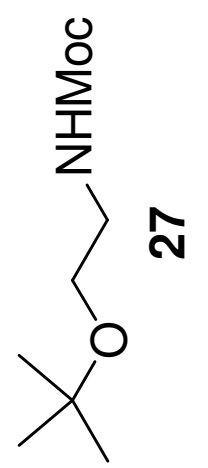


$88 t^{\circ} L Z$

$6 เ 9 \bullet$

$\angle 200^{\circ}$

089.09

$690^{\circ} \varepsilon L$
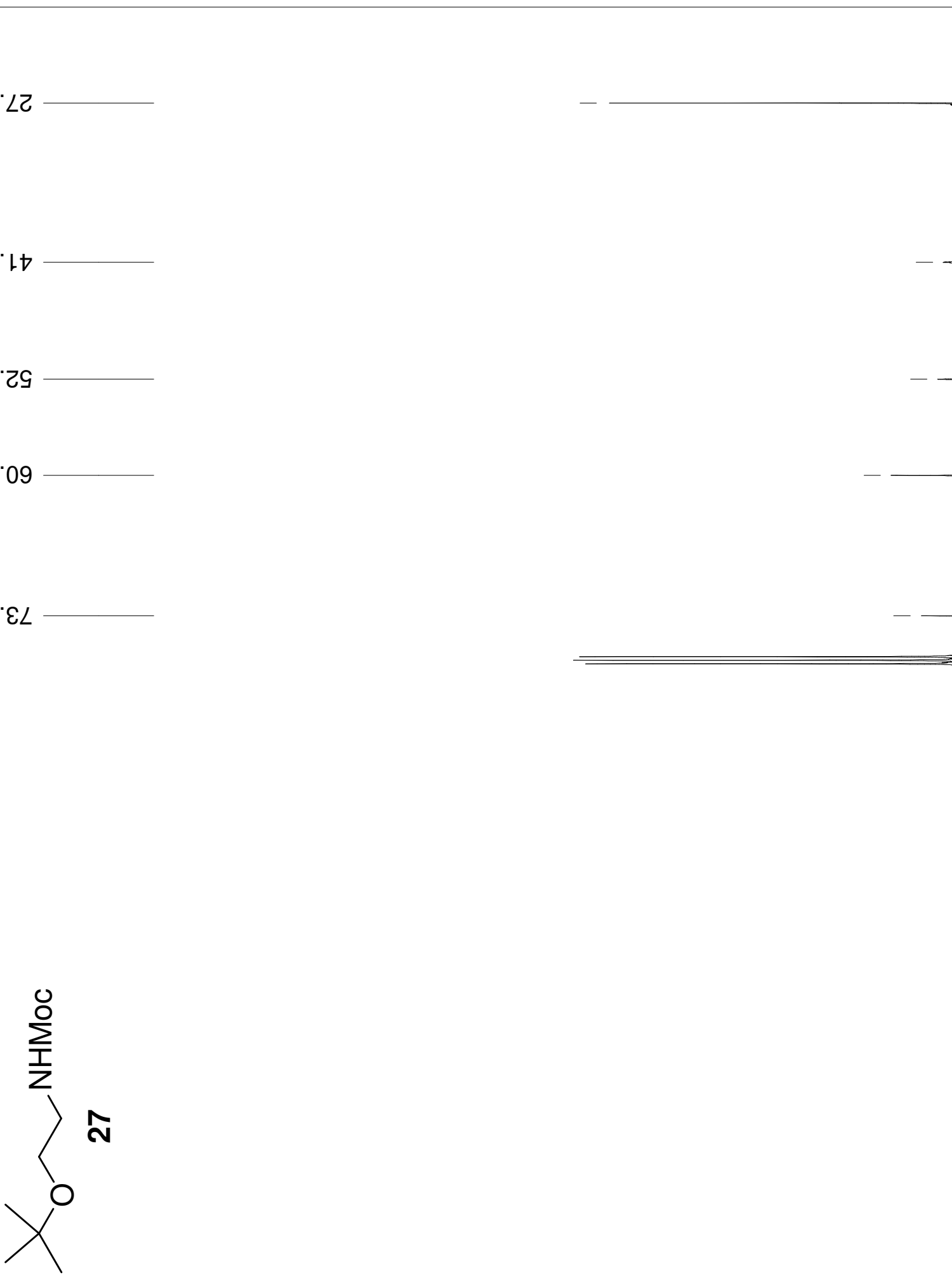

$\nabla 60^{\circ} \angle G$ 


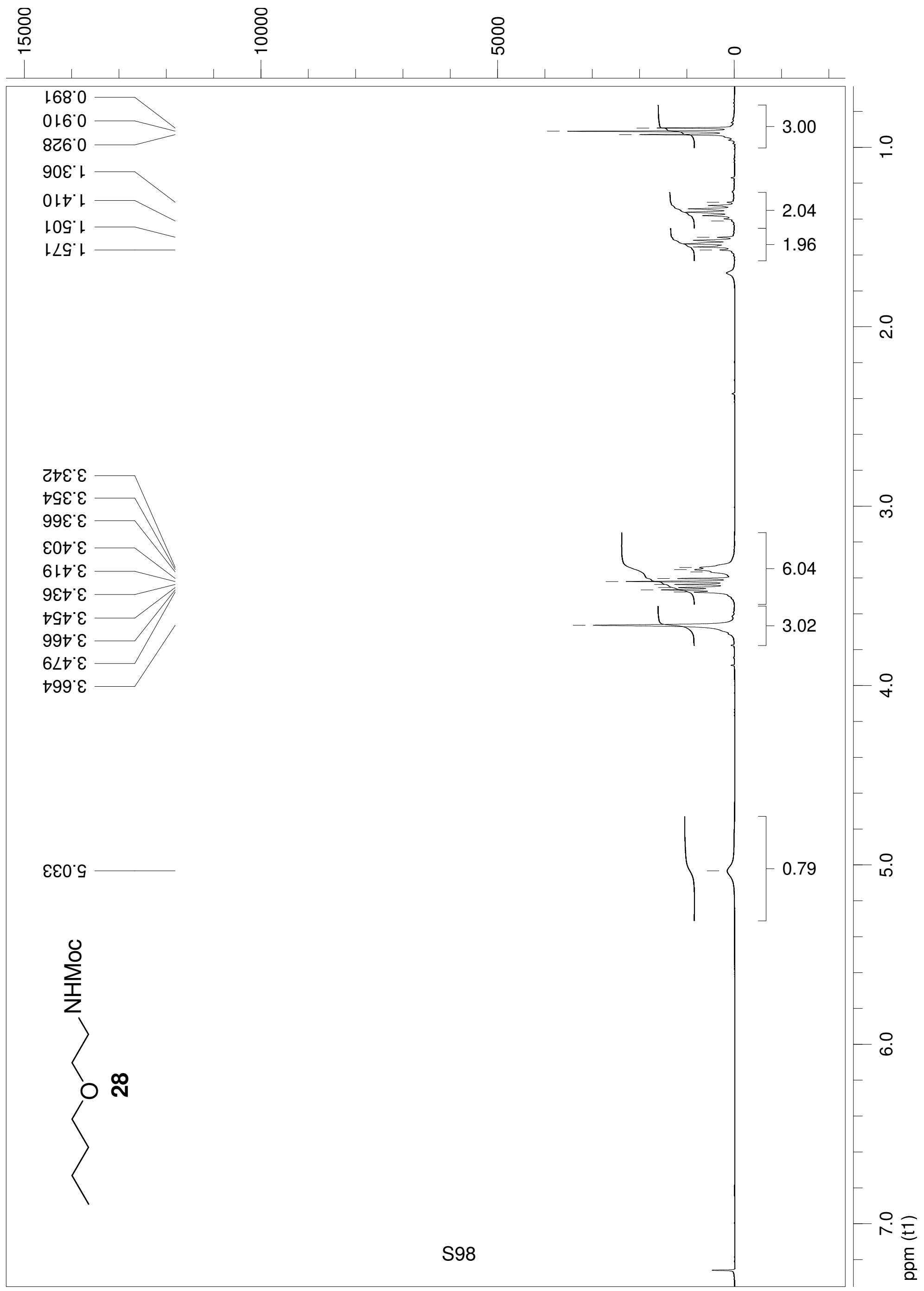




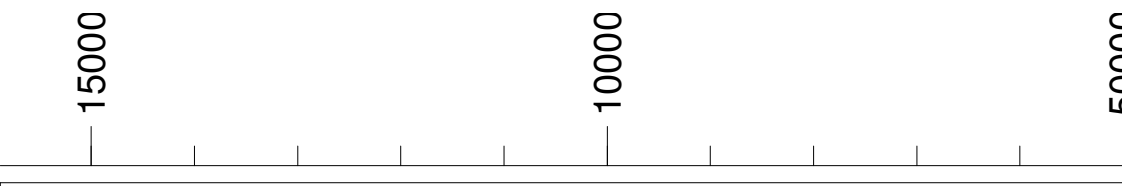

亏

SG8' $\varepsilon$

$\nabla \angle Z^{\prime} 6$ L

$\dashv 69^{\circ} \mid \mathcal{E}$

L00.

†८०२৭

$69 t \times 69$

$096^{\circ} 0 L$
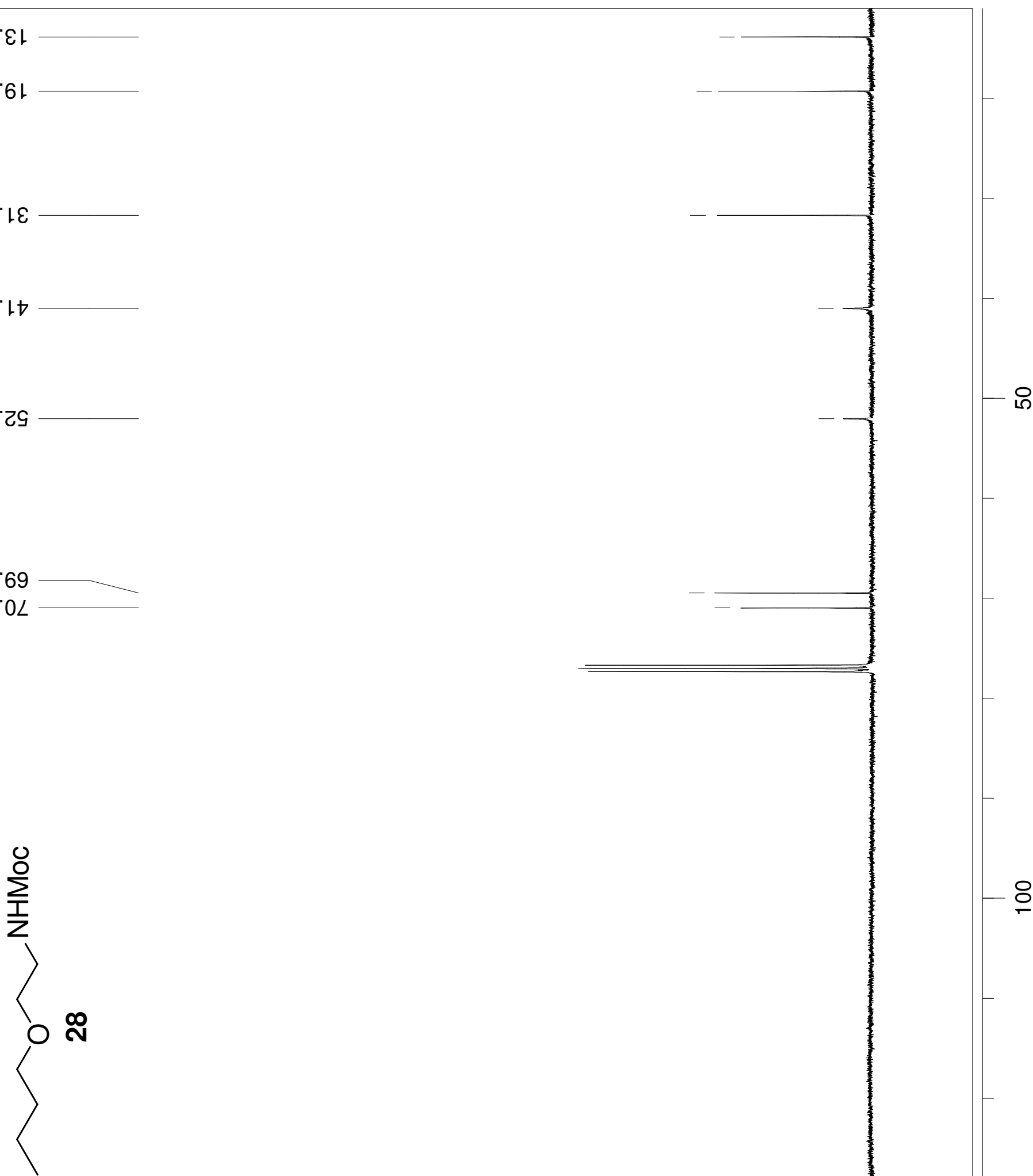

$\stackrel{8}{\circ}$ 


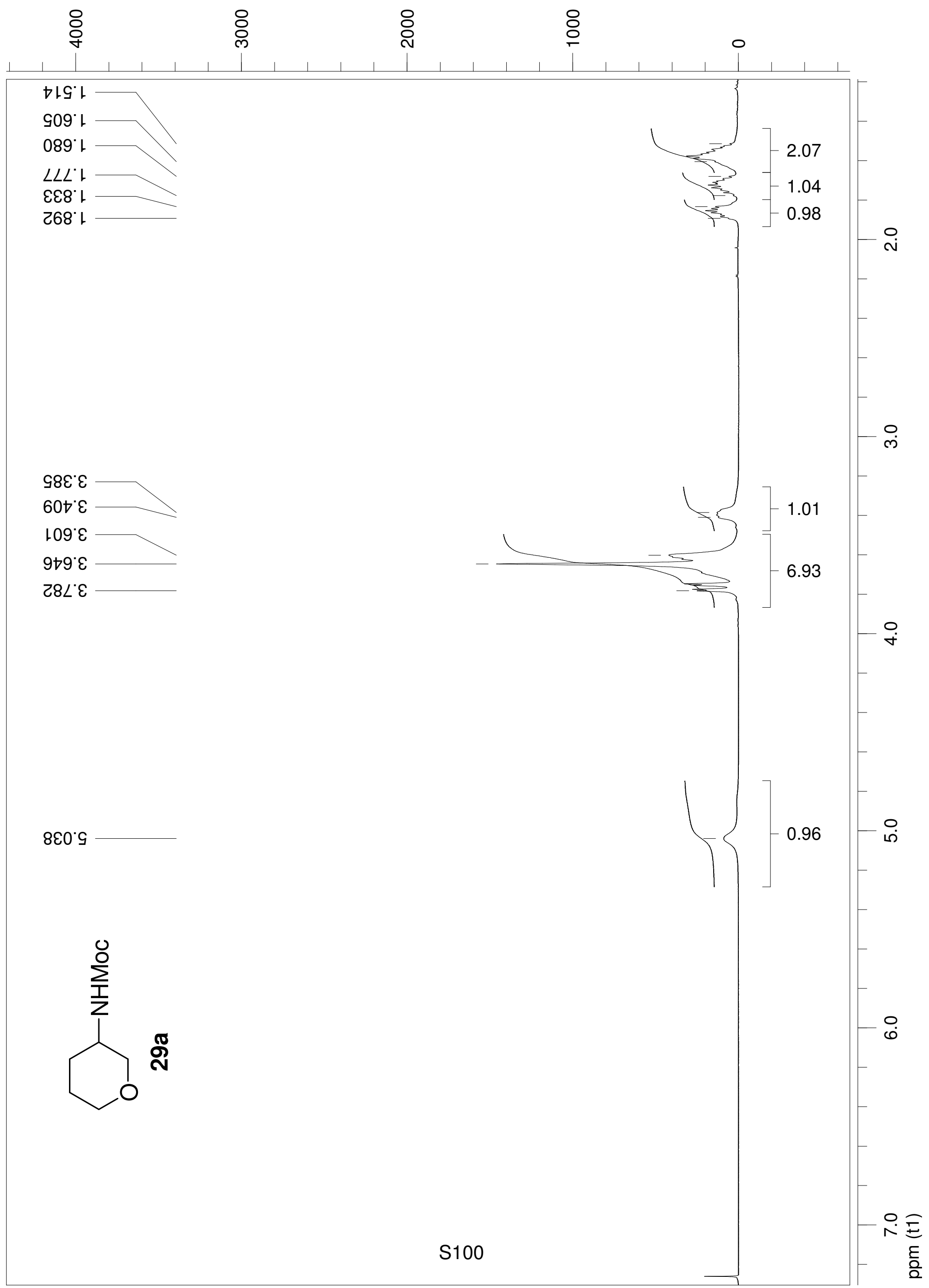


,

$\frac{8}{8}$

g

응

$660^{\circ} \varepsilon 己$

$\angle 98^{\circ} 82$

sงt:9t

LE6 'L

220.89

LItトL

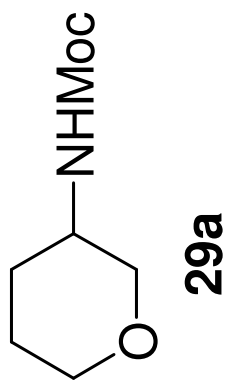




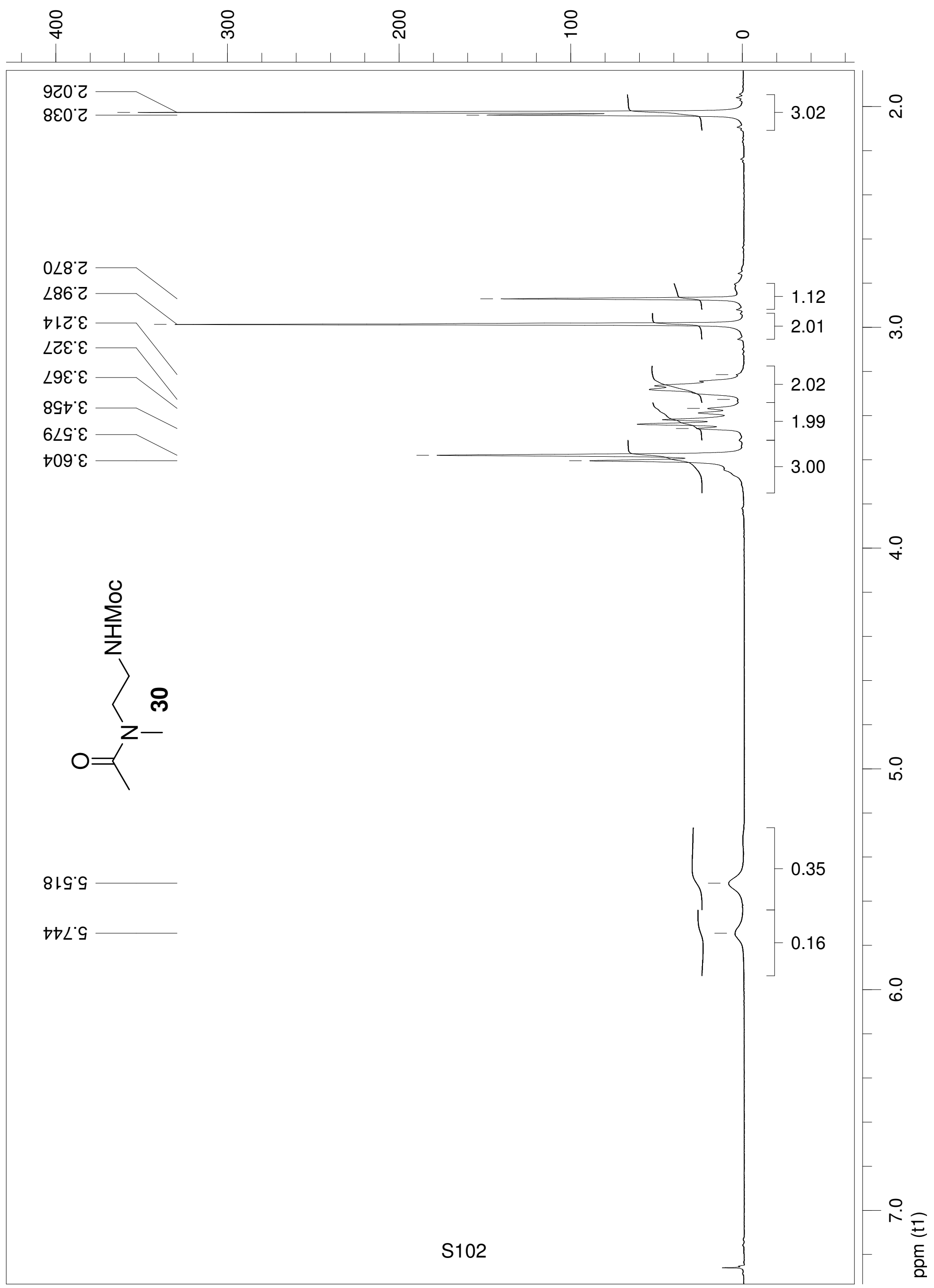




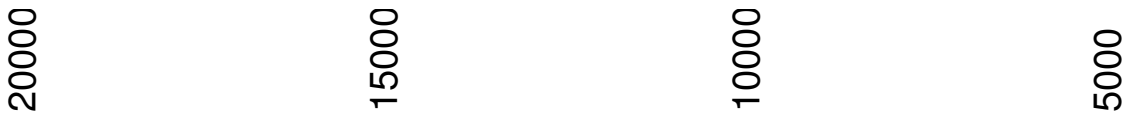

$896^{\circ} 02$

$\varepsilon \varepsilon 9^{\circ}$ เ乙

ट9. $\varepsilon \varepsilon$

$\angle 19^{\circ} 9 \varepsilon$

$8 \vdash 8^{\circ} 8 \varepsilon$

$991^{\circ} 6 \varepsilon$

$1 \angle 6.9 t$

$\downarrow 26^{\circ} 6 \mathrm{t}$

$\succ 88^{\circ} \mathrm{LS}$

$\angle \angle 0^{\circ} \mathrm{ZS}$

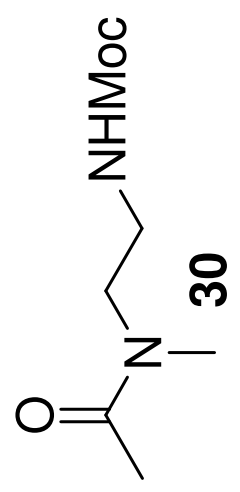

† เ $\angle G \mid$

$\angle 9 Z^{\circ} \angle G$ L 
亏

ร

$\mid \angle 6^{\circ}$

$166^{\circ}$ เ

010 己

$120^{\circ} \mathrm{Z}$

$620^{\circ} 2$

$\angle \triangleright 0^{\circ} \mathrm{C}$

6ยะ'ح

$69 \varepsilon^{\circ} 2$

6Lع 乙

9२ह $\varepsilon$

8हย' $\varepsilon$

$0 \angle \varepsilon^{\circ} \varepsilon$

५8.' $\varepsilon$

$86 \varepsilon^{\circ} \varepsilon$

乙เฑ $\varepsilon$

oहt $\varepsilon$

$\angle \nabla \nabla^{\circ} \varepsilon$

$0 乙 99^{\circ} \varepsilon$

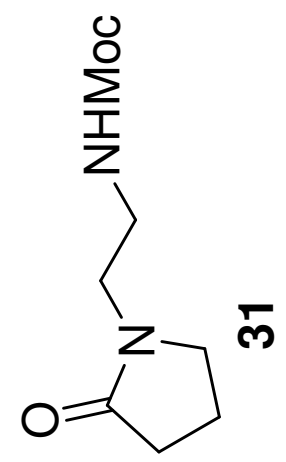

$962^{\circ} 9$

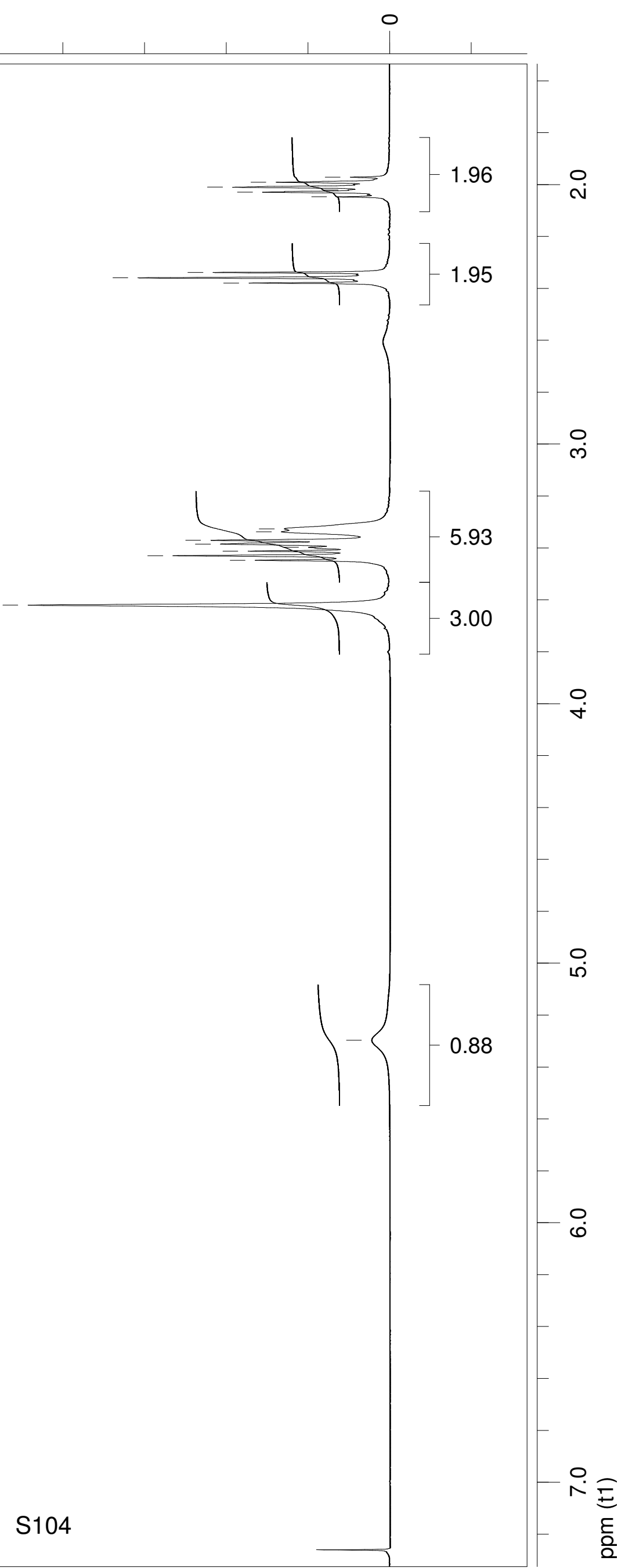




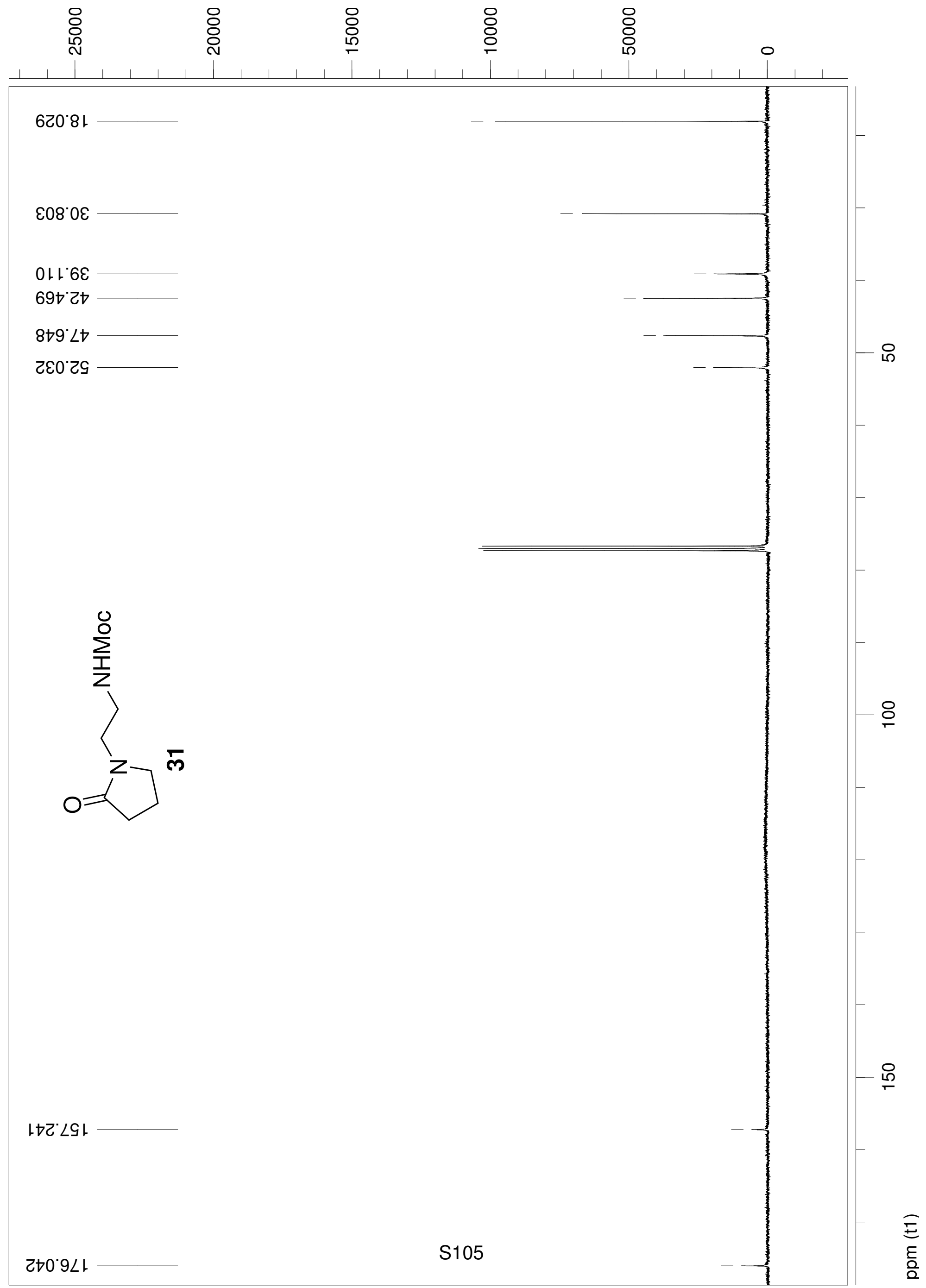




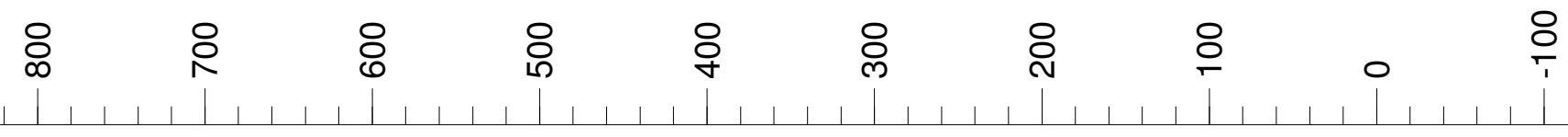

699' เ

$\angle E L ' L$

$0 \angle t C$

$\angle O S^{\circ} \mathrm{C}$

6 เट' $\varepsilon$

$8 L \mathcal{C}^{\cdot} \varepsilon$

$\angle 6 \mathcal{C}^{\circ} \varepsilon$

9๕.' $\varepsilon$

$6 \nabla \varepsilon^{\circ} \varepsilon$

$89 \varepsilon^{\circ} \varepsilon$

เง๋ $\varepsilon$

ट $\angle{ }^{\circ} \varepsilon$

ع6ฑ $\varepsilon$

$\mathrm{S}^{\circ} \mathcal{}{ }^{\circ} \varepsilon$

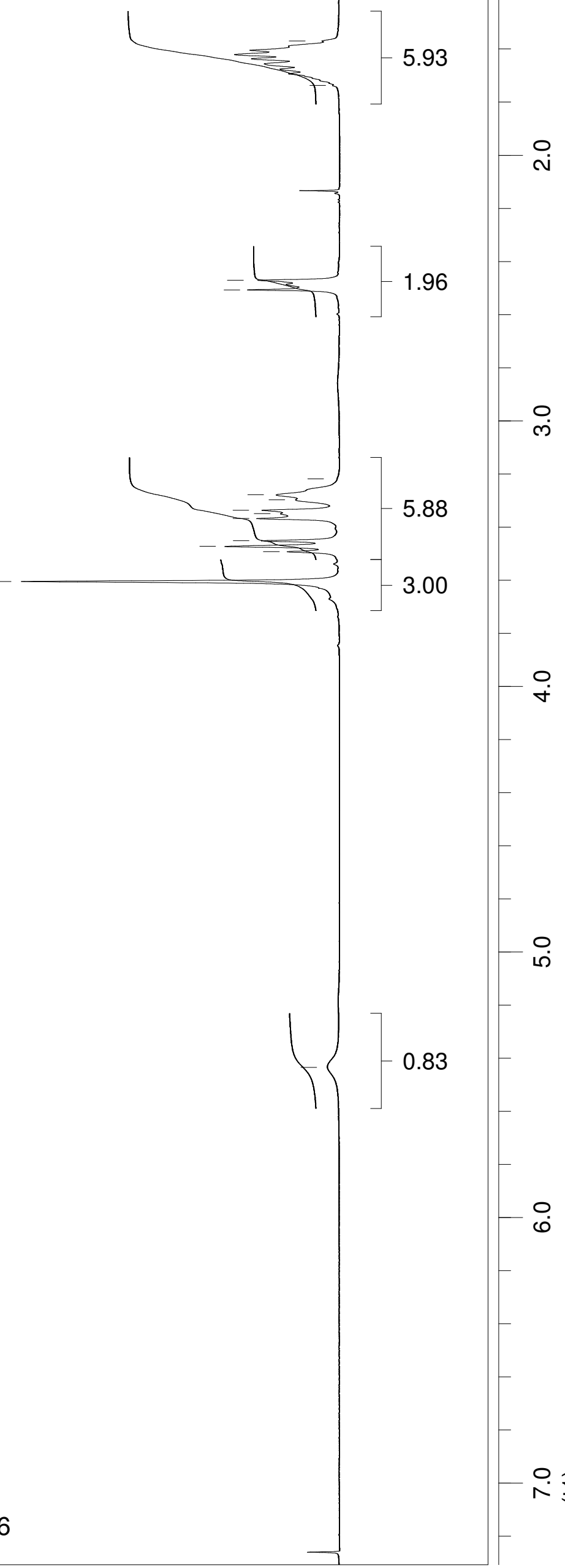

†ยฑ

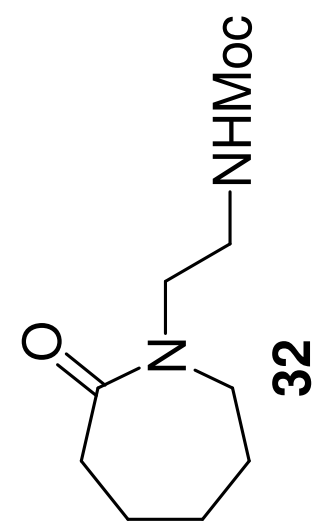


亏

ঃ

6๖乙 $๕ 己$

$86 \varepsilon^{\circ} 82$

$008^{\circ} 62$

$\mathrm{S} \angle 6^{\circ} 9 \varepsilon$

$0200^{\circ} 0 t$

$669^{\circ} \angle t$

टGट 09

ट†6.।S

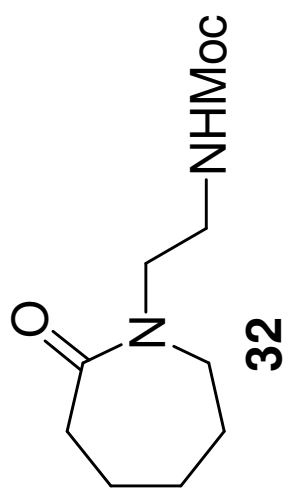

$\vdash \angle Z \angle G$ 
웅

เOt $\varepsilon$

$929^{\circ} \varepsilon$

$269^{\circ} \varepsilon$

$\angle 8 L^{\prime} \varepsilon$

$988^{\circ} \varepsilon$

요

$\angle \nabla \vdash \cdot 9$

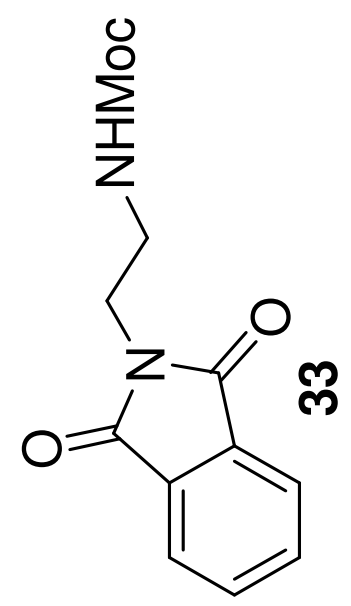

$6 \angle 9^{\circ} \angle$

$\angle E L ' L$

$88 L^{\circ} L$

$098^{\circ} L$ 
总

ะ

$\angle L L ' \angle E$

8LC $0 t$

IGเZG

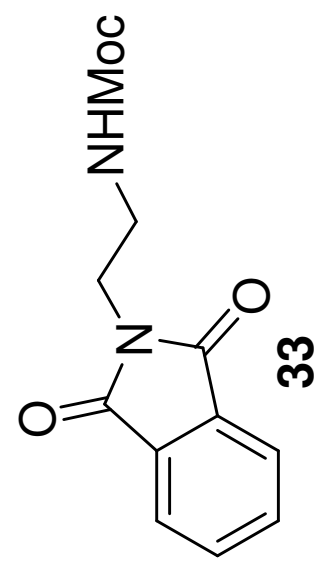

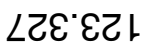

เฤ8 เยเ

$\triangleright \succsim 0^{\circ} \nabla \varepsilon$

$\downarrow 20^{\circ} \angle S+$

Gટt789เ 